\title{
Generating Datasets for Anomaly-Based Intrusion Detection Systems in IoT and Industrial IoT Networks
}

\author{
Ismael Essop ${ }^{1}$, José C. Ribeiro ${ }^{2, *}$, Maria Papaioannou ${ }^{1,2}{ }^{\circledR}$, Georgios Zachos ${ }^{1,2}$, Georgios Mantas ${ }^{1,2}$ and \\ Jonathan Rodriguez ${ }^{2,3}$ \\ 1 Faculty of Engineering and Science, University of Greenwich, Chatham Maritime ME4 4TB, UK; \\ i.a.essop@gre.ac.uk (I.E.); m.papaioannou@av.it.pt (M.P.); g.zachos@gre.ac.uk (G.Z.); gimantas@av.it.pt (G.M.) \\ Instituto de Telecomunicações, Aveiro 3810-193, Portugal; jonathan@av.it.pt \\ 3 Faculty of Computing, Engineering and Science, University of South Wales, Pontypridd CF37 1DL, UK \\ * Correspondence: jcarlosvgr@av.it.pt
}

Citation: Essop, I.; Ribeiro, J.C.;

Papaioannou, M.; Zachos, G.; Mantas,

G.; Rodriguez, J. Generating Datasets

for Anomaly-Based Intrusion

Detection Systems in IoT and

Industrial IoT Networks. Sensors 2021,

21, 1528. https://doi.org/10.3390/

s21041528

Academic Editor: David Plets

Received: 8 January 2021

Accepted: 18 February 2021

Published: 23 February 2021

Publisher's Note: MDPI stays neutral with regard to jurisdictional claims in published maps and institutional affiliations.

Copyright: (c) 2021 by the authors. Licensee MDPI, Basel, Switzerland. This article is an open access article distributed under the terms and conditions of the Creative Commons Attribution (CC BY) license (https:/ / creativecommons.org/licenses/by/ $4.0 /)$.

\begin{abstract}
Over the past few years, we have witnessed the emergence of Internet of Things (IoT) and Industrial IoT networks that bring significant benefits to citizens, society, and industry. However, their heterogeneous and resource-constrained nature makes them vulnerable to a wide range of threats. Therefore, there is an urgent need for novel security mechanisms such as accurate and efficient anomaly-based intrusion detection systems (AIDSs) to be developed before these networks reach their full potential. Nevertheless, there is a lack of up-to-date, representative, and well-structured IoT/IIoT-specific datasets which are publicly available and constitute benchmark datasets for training and evaluating machine learning models used in AIDSs for IoT/IIoT networks. Contribution to filling this research gap is the main target of our recent research work and thus, we focus on the generation of new labelled IoT/IIoT-specific datasets by utilising the Cooja simulator. To the best of our knowledge, this is the first time that the Cooja simulator is used, in a systematic way, to generate comprehensive IoT/IIoT datasets. In this paper, we present the approach that we followed to generate an initial set of benign and malicious IoT/IIoT datasets. The generated IIoT-specific information was captured from the Contiki plugin "powertrace" and the Cooja tool "Radio messages".
\end{abstract}

Keywords: IoT; Industrial IoT; benign datasets generation; malicious datasets generation; Cooja simulator; Contiki OS; anomaly-based intrusion detection

\section{Introduction}

Despite the significant benefits that IoT and Industrial IoT (IIoT) networks bring to citizens, society, and industry, the fact that these networks incorporate a wide range of different communication technologies (e.g., WLANs, Bluetooth, and Zigbee) and types of nodes/devices (e.g., temperature/humidity sensors), which are vulnerable to various types of security threats, raises many security and privacy challenges in IoT/IIoT-based systems. For instance, attackers may compromise IoT/IIoT networks in order to manipulate sensing data (e.g., by injecting fake data) and cause malfunction to the IoT/IIoT-based systems that rely on the compromised IoT/IIoT networks. It is worthwhile to mention that IoT/IIoT networks can become an attractive target of attackers with a wide spectrum of motivations ranging from criminal intents aimed at financial gain to industrial espionage and cybersabotage. Therefore, security solutions protecting IoT/IIoT networks from attackers are critical for the acceptance and wide adoption of such networks in the coming next years. Nevertheless, the high resource requirements of complex and heavyweight conventional security mechanisms cannot be afforded by (i) the resource-constrained IoT/IIoT nodes (e.g., sensors) with limited processing power, storage capacity, and battery life; and/or (ii) the constrained environment in which the nodes are deployed and interconnected using lightweight communication protocols. Consequently, there is an urgent need for novel security mechanisms, such as accurate and efficient anomaly-based intrusion detection systems 
(AIDSs) tailored to the resource-constrained characteristics of IoT/IIoT networks, to be developed in order to address the pressing security challenges of IoT/IIoT networks with reasonable cost, in terms of processing and energy, before IoT/IIoT networks gain the trust of all involved stakeholders and reach their full potential in the market [1-3]. However, there is a lack of up-to-date, representative and well-structured IoT/IIoT-specific datasets that are publicly available to the research community and constitute benchmark datasets for training and evaluating machine learning (ML) models used in AIDSs for IoT/IIoT networks $[4,5]$. This lack of benchmark IoT/IIoT datasets constitutes a significant research gap that should be addressed in order to develop more accurate and efficient IoT/IIoT-specific AIDS whose effectiveness is evaluated based on their performance to detect IoT/IIoT attacks which is a process reliant on comprehensive IoT/IIoT-specific datasets.

In fact, although several datasets, such as KDDCUP99 [6], NSL-KDD [7], UNSWNB15 [8], and CICD2017 [9] have been created over the past two decades for evaluation purposes of network-based intrusion detection systems (IDSs), they do not include any specific characteristics of IoT/IIoT networks as these datasets do not contain sensors' reading data or IoT/IIoT network traffic $[4,5]$. To respond to this major issue, few efforts focused on the generation of IoT-specific datasets have also been seen in the literature recently. However, they are characterised by some limitations in terms of the IoT-specific information they include. For instance, the datasets proposed in $[10,11]$ are IoT-specific datasets but they lack of events reflecting attack scenarios. To address this limitation, the IoT-specific and network-related datasets proposed in $[12,13]$ contain events reflecting attack scenarios; however, they do not cover a diverse set of attack scenarios and do not include sensors' reading data or information related to the behaviour of the IoT/IIoT devices (e.g., sensors/actuators) within the network. Therefore, these IoT datasets can mainly be used for detecting only a limited number of network-based attacks against IoT/IIoT networks as they do not contain adequate information for detecting a wide range of network-based attacks and/or attacks that manipulate sensor measurement data or compromise IoT/IIoT devices within the IoT/IIoT network.

Consequently, there is an urgent need for comprehensive IoT/IIoT-specific datasets containing not only network-related information (e.g., packet-level information and flowlevel information) but also events reflecting multiple benign and attack scenarios from current IoT/IIoT network environments, sensor measurement data, and information related to the behaviour of the IoT/IIoT devices deployed within the IoT/IIoT network for efficient and effective training and evaluation of AIDSs suitable for IoT/IIoT networks. Towards this direction, the recent work of [4] has proposed, for the first time, to the best of our knowledge, a new dataset that includes events of a variety of IoT-related attacks and legitimate scenarios, IoT telemetry data collected from heterogeneous IoT/IIoT data sources, network traffic of IoT/IIoT network, and audit traces of operating systems [4]. Therefore, it is clear that more comprehensive IoT/IIoT-specific datasets including events reflecting multiple benign and attack scenarios, sensor measurement data, network-related information, and information related to the behaviour of the IoT/IIoT devices are required to be generated and become publicly available to the research community so as to fill this significant research gap of lack of benchmark IoT/IIoT datasets and more accurate and efficient IoT/IIoT-specific AIDS to be developed.

Contribution to filling this research gap is the main target of our recent research work. In particular, our focus is on the generation of new labelled IoT/IIoT datasets that will be publicly available to the research community and include: (a) events reflecting multiple benign and attack scenarios from current IoT/IIoT network environments, (b) sensor measurement data, (c) network-related information (e.g., packet-level information and flow-level information) from the IoT/IIoT network, and (d) information related to the behaviour of the IoT/IIoT devices deployed within the IoT/IIoT network. It is worthwhile to mention that the new labelled IoT/IIoT datasets are generated by implementing various benign IoT/IIoT network scenarios and IoT/IIoT network attack scenarios in the Cooja simulator which is the companion network simulator of the open source Contiki Operating 
System (OS) that is one of the most popular OSs for resource constrained IoT devices [14]. To the best of our knowledge, this is the first time that the Cooja simulator is going to be used, in a systematic way, to generate comprehensive IoT/IIoT datasets. In this paper, we present the approach that we followed to generate an initial set of benign IoT/IIoT datasets (i.e., including only normal events) and malicious IoT/IIoT datasets (i.e., including attack and normal events) by utilising the Cooja simulator that was the simulation environment where the corresponding benign and attack scenarios were implemented.

The rest of this paper is organised as follows. In Section 2, the main threats against the IoT/IIoT network (i.e., perception domain) are presented and in Section 3, examples of anomaly-based intrusion detection systems for IoT/IIoT networks are discussed. In Section 4, a detailed description of the approach followed to generate a set of benign datasets by implementing a benign IIoT network scenario in the Cooja simulator is provided. In Section 5, a detailed description of the approach followed to generate a set of malicious datasets by implementing a User Datagram Protocol (UDP) flooding attack scenario in the Cooja is provided as well. In Section 6, a discussion on the generated datasets is given. Finally, Section 7 concludes this paper.

\section{Threat Analysis of the IoT/IIoT Network (Perception Domain)}

The perception domain, as shown in Figure 1, can be perceived as the device layer in the ITU-T reference model [15]. As the main purpose of the perception domain is to gather data, the security challenges in this domain target to forge collected IoT/IIoT data and damage perception devices, as presented below.

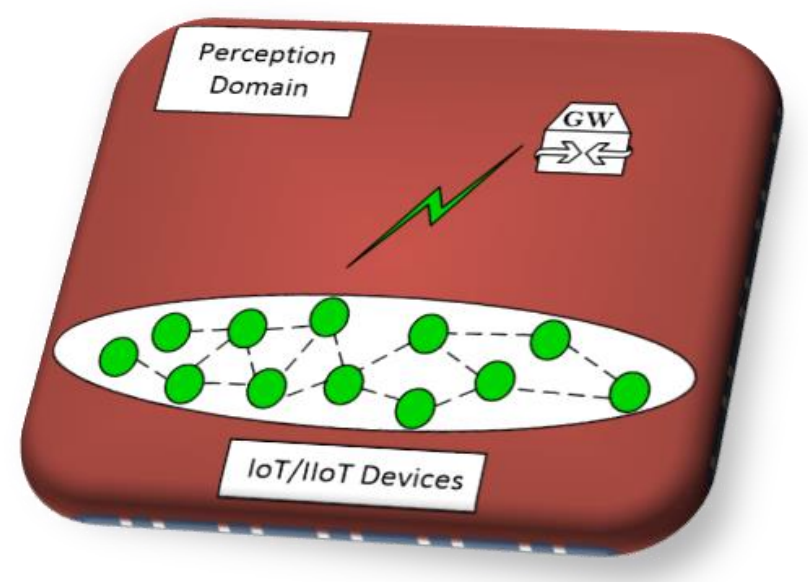

Figure 1. IoT/IIoT Network (Perception Domain).

\subsection{Sinkhole Attacks}

In this type of attacks, a compromised IoT/IIoT node (i.e., IoT/IIoT gateway [16]) in the perception domain proclaims very appealing capabilities of power, computation and communication [17] so that nearby nodes (i.e., IoT/IIoT sensors) will choose it as the forwarding node in the routing process due to its very attractive capabilities. As a consequence, the compromised IoT/IIoT node can increase the amount of data obtained before it is delivered to the cloud domain of the IoT-based monitoring system. Therefore, a sinkhole attack can not only compromise the confidentiality of the manufacturing data but also can comprise an initial step to launch additional attacks such as DoS/DDoS attacks $[17,18]$.

\subsection{Node Capture Attacks}

In this type of attack, the adversary is able to extract important information about the captured node, such as the group communication key, radio key, etc. [17]. Additionally, the adversary can copy the important information related to the captured node to a malicious node, and afterwards fake the malicious node as a legitimate node to connect to the 
IoT/IIoT network (i.e., perception domain). This type of attack is also known as node cloning/replication attack $[17,19]$. This attack may lead to compromising the security of the complete IoT/IIoT-based monitoring system.

\subsection{Malicious Code Injection Attacks}

An attacker can take control of an IoT/IIoT node or device in the perception domain by exploiting its security vulnerabilities in software and hardware and injecting malicious code into its memory. Afterwards, using the malicious code, the attacker can force the node or device to perform unintended operations. For example, the infected IoT/IIoT node(s) or device(s) can be used as a bot(s) to launch further attacks (e.g., DoS and DDoS) against other devices or nodes within the perception domain or even against the other domains (i.e., Network domain and Cloud domain). In addition, the attacker can use the injected malicious code in the infected device or node to get access into the IoT/IIoT-based system and/or get full control of the system [19].

\subsection{False Data Injection Attacks}

After capturing an IoT/IIoT node or device in the perception domain, the adversary can inject false data in place of benign data measured by the captured IoT/IIoT node or device and transmit the false data to the Cloud domain [17]. Thereafter, receiving the false data, the IoT/IIoT-based system may provide wrong services, which further negatively impacts the effectiveness of system itself.

\subsection{Replay Attacks}

In the perception domain, the attacker can use a malicious IoT/IIoT node or device to transmit to the destination host (i.e., IoT/IIoT gateway) with legitimate identification information, already received by the destination host, so that the malicious node or device can become a trusted node/device to the destination host [17]. Replay attacks are commonly launched in authentication process to destroy the validity of certification.

\subsection{Eavesdropping}

As the IoT/IIoT nodes and devices in perception domain communicate via wireless networks, an attacker (i.e., eavesdropper) can retrieve sensitive manufacturing data by overhearing the wireless transmission. For instance, an adversary within the perception domain can eavesdrop exchanged information by tracking wireless communications and reading the contents of the transmitted packages [17]. The eavesdropper can passively intercept the wireless communication between a sensor (e.g., environment industrial sensors or sensors on the machine resources) and the IoT/IIoT gateway, and extract confidential data (e.g., through traffic analysis) in order to maliciously use them.

\subsection{Sleep Deprivation Attacks or Denial of Sleep Attacks}

These attacks target to drain the battery of the resource constrained IoT/IIoT devices of the perception domain. In principle, the IoT/IIoT devices in the perception domain are usually programmed to follow a sleep routine when they are inactive in order to reduce the power consumption and extend their life cycle. However, an adversary may break the programmed sleep routines and keep the IoT/IIoT devices of the perception domain continuously active until they are shut down due to a drained battery. Attackers can achieve this by running infinite loops in these devices using malicious code or by artificially increasing their power consumption [20].

\subsection{Sybil Attacks}

In a sybil attack, a malicious or sybil node or device can illegitimately claim multiple identities, allowing it to impersonate them within the perception domain. For instance, the malicious node can achieve to connect with several other devices in order to maximise its influence and even deceive the complete system to draw incorrect conclusions [21]. 


\subsection{Denial of Service (DoS) Attacks}

The main target of these attacks is to deplete resources of the perception domain in order to make the whole IoT/IIoT network or specific nodes (e.g., machine or/and environment resources) or devices (e.g., IoT/IIoT gateway) unavailable. For instance, jamming attacks are a type of DoS attacks where an attacker transmits a high-range signal to overload the communication channel between two communicating entities and disrupt their communication. Within the perception domain of the IoT/IIoT-based system, jamming attacks can disrupt the communication between the IoT/IIoT sensors and the Gateway in order to prevent data from being transmitted to the Gateway, leading to malfunctions in the provided services to the authorised users. Jamming attacks can be performed by passively listening to the wireless medium so as to broadcast on the same frequency band as the legitimate transmitting signal. Finally, distributed denial of service (DDoS) attacks are a large-scale variant of DoS attacks and in the case of the perception domain an example of DDoS attack is when a large number of nodes (e.g., IoT/IIoT sensors) are compromised so as to flood the Gateway with a lot of transmitted data/requests and render it unavailable or disrupt its normal operations [22,23].

\section{Anomaly-Based Intrusion Detection Systems for IoT/IIoT Networks}

In this Section, two examples of anomaly-based intrusion detection systems for IoT/IIoT networks are discussed. Moustafa et al. in [24] proposed an ensemble network intrusion detection technique which utilises established statistical flow features. The goal is to mitigate malicious events, and more specifically botnet attacks against DNS, HTTP and MQTT protocols that are employed in IoT networks. The first step of their work revolves around the deep analysis of the TCP/IP model and the subsequent extraction of a set of features from the network traffic protocols MQTT, HTTP, and DNS protocols. The Bro-IDS tool is used by the authors for basic features while they also employ, in parallel, their own extractor module to generate additional statistical features of the transactional flows. Consequently, features are filtered and only the most important ones are selected in order to simplify the NIDS and decrease its computational cost. In this step, the authors utilise the correlation coefficient on result features as a means of features selection. Lastly, an AdaBoost ensemble learning method is developed to detect the attacks. The method is based on the combination of three different Machine Learning (ML) algorithms; decision tree (DT), Naive Bayes (NB), and artificial neural network (ANN) algorithms. These classification techniques were chosen mainly due to the core entropy measure that was calculated from the feature vectors. The AdaBoost (Adaptive Boosting) method improves the performance of the detection in comparison to using each machine learning algorithm separately. In case of small differences of the feature vectors, an error function is employed. The importance of the error function lies in computing the error value for each instance of the distributed input data. Based on this error value, it is possible to understand and evaluate which learners are best suited to classify each instance. The experiments results show that the ensemble technique achieved a high detection rate $(95.25 \%-99.86 \%)$ and a low false positive rate (between $0.01 \%$ and $0.72 \%$ ) compared to existing state-of-the-art techniques. The authors employed the UNSWNB15 and NIMS botnet datasets with simulated IoT sensor data to support their findings.

Furthermore, a multi-layer perceptron (MLP), which is a type of supervised artificial neural network [25]), is used in an offline IDS for IoT networks [26]. The ANN consists of 3 layers and each of the hidden and output layers' neurons use a unipolar sigmoid transfer function to transform their input values to a specific output value. The network was trained using a stochastic learning algorithm with mean square error function. The training process included both feed-forward and backward training algorithms. To perform its task, the ANN analyses the Internet packet traces and attempts to detect DoS and DDoS attacks in IoT network. In order to evaluate the IoT IDS, an experimental architecture was created with four client nodes and a server relay node. The server node was subjected not only to DOS attacks from a single host with more than 10 million UDP packets sent but 
also to DDoS attacks from three hosts each sending over 10 million UDP packets at wire speed. The results of their simulations showed a detection accuracy of $99.4 \%$ and $0.6 \%$ false positive rate. The authors used a training dataset consisting of a total of 2313 samples, 496 of them deployed for validation and 496 of them for testing [5].

\section{Generation of Benign IoT/IIoT Datasets}

In this Section, we provide a detailed description of the approach followed to generate a set of benign datasets by implementing a benign IoT/IIoT network scenario in the Cooja simulator, as shown in Figure 2. The generated IoT/IIoT-specific information from the simulated scenario was captured from the Contiki plugin "powertrace" (i.e., features such as CPU consumption) and the Cooja tool "Radio messages" (i.e., network traffic features) in order to generate the "powertrace" dataset and the network traffic dataset for the simulated benign IoT/IIoT network scenario.

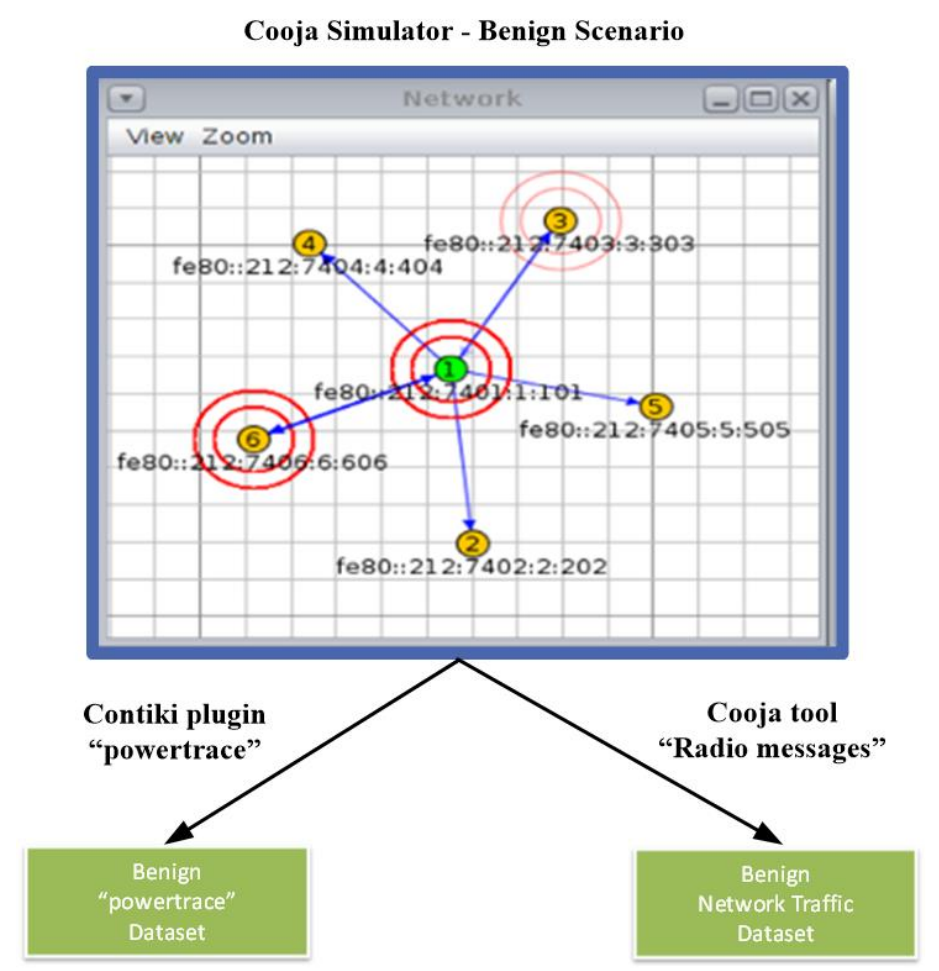

Figure 2. Benign datasets generation by utilizing the Cooja simulator.

The network topology of the simulated benign IoT/IIoT network scenario in the Cooja simulator environment consists of 5 yellow UDP-client motes (i.e., motes 2, 3, 4, 5 and 6) and the green UDP-server mote (i.e., mote 1), as depicted in Figure 2. The simulation duration was set to $60 \mathrm{~min}$ and the motes' outputs were printed out in the respective window (e.g., Mote output) while simulations run, as shown in Figure 3. In addition, the yellow UDP-client motes were configured to send text messages every $10 \mathrm{~s}$, approximately, to the green UDP-sever mote that was configured to provide a corresponding response. The UDP protocol was used at the Transport Layer and the IPv6 at the network layer. Moreover, the type of motes used in this scenario was the Tmote Sky that is an ultralow power wireless module for use in sensor networks, monitoring applications, and rapid application prototyping. In addition, Tmote Sky motes leverage industry standards such as USB and IEEE 802.15.4 to interoperate seamlessly with other devices. By using industry standards, integrating humidity, temperature, and light sensors, and providing flexible interconnection with peripherals, Tmote Sky motes enable several mesh network applications [27]. 


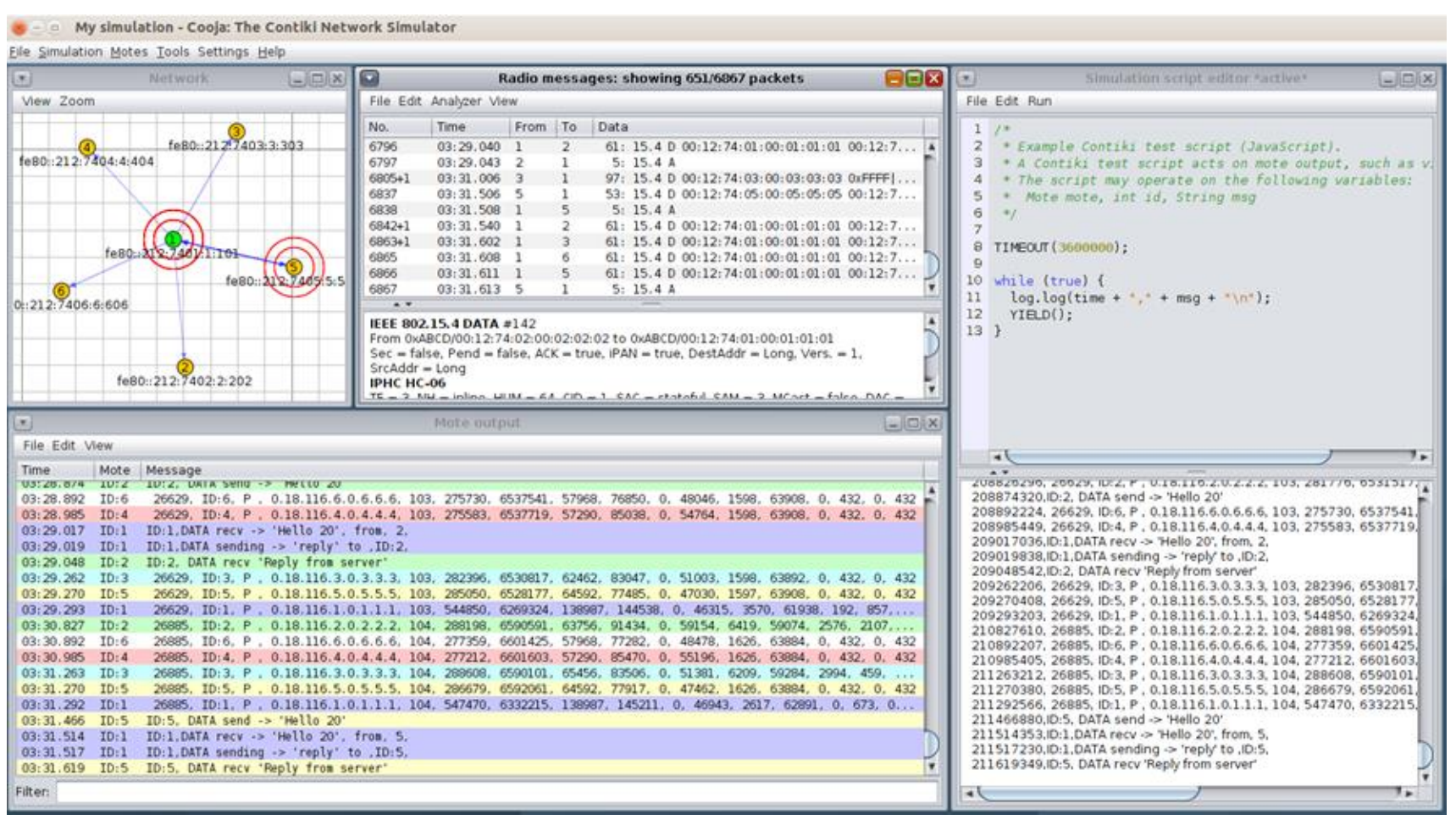

Figure 3. Cooja Simulator-motes' outputs.

\subsection{Benign "Powertrace" Dataset Generation}

\subsubsection{Benign "Powertrace" Dataset Generation}

The "powertrace" dataset includes information about features such as total CPU energy consumption and low power mode (LPM) energy consumption. In fact, it is the dataset of the simulated benign IIoT network scenario that includes records about information related to the energy consumption of the IIoT devices (i.e., motes) deployed within the simulated IIoT network. To enable the "powertrace" plugin and generate the "powertrace" dataset, we programmed the motes of the benign IIoT network to make use of the "powertrace" plugin for collecting "powertrace" related features every 2 s. In particular, we included the "powertrace.h" library into the code of each mote (i.e., \#include "powertrace.h"), as shown in Figure 4, and defined to start powertracing, once every $2 \mathrm{~s}$, in the code of each mote as shown in Figure 5.

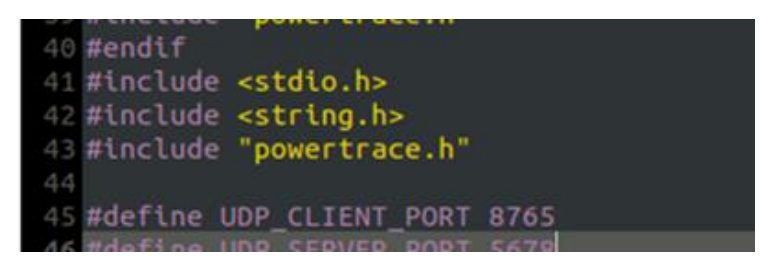

Figure 4. "powertrace.h" library in the mote code.

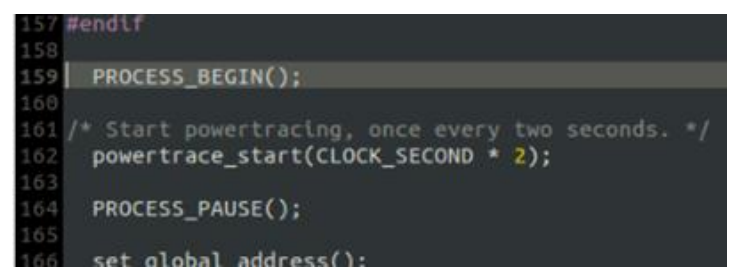

Figure 5. Powertracing Begin. 
More precisely, the "powertrace" plugin captured raw information, every $2 \mathrm{~s}$, about the set of features summarised in Table 1. In particular, the "powertrace" plugin tracks the duration (i.e., number of cpu ticks) of activities of a mote being in each power state. Particularly, the outputs demonstrate the fraction of time in which a mote remains for a given power state. There are the following six power states: (i) $\mathrm{cpu}$; (ii) lpm; (iii) transmit; (iv) listen; (v) idle_transmit; and (vi) idle_listen, as shown in Table 1. These are measured with a hardware timer (i.e., clock frequency is defined in RTIMER_SECOND or 32,768 Hz for XM1000).

Table 1. "powertrace" plugin-Set of Captured Features.

\begin{tabular}{ccc}
\hline Index & Feature & Description \\
\hline 1 & sim time & simulation time \\
\hline 2 & clock_time() & clock time (i.e.,by default, 128 ticks/second) \\
\hline 3 & ID & Mote ID \\
\hline 4 & P & label \\
\hline 5 & rimeaddr & rime address \\
\hline 6 & seqno & sequence number \\
\hline 7 & all_cpu & accumulated CPU energy consumption \\
\hline 8 & all_lpm & accumulated transmission energy consumption \\
\hline 9 & all_transmit & accumulated listen energy consumption \\
\hline 10 & all_listen & accumulated idle transmission energy consumption \\
\hline 11 & all_idle_transmit & accumulated idle listen energy consumption \\
\hline 12 & all_idle_listen & CPU energy consumption for this cycle \\
\hline 13 & cpu & LPM energy consumption for this cycle \\
\hline 14 & lpm & transmission energy consumption for this cycle \\
\hline 15 & transmit & listen energy consumption for this cycle \\
\hline 16 & listen & idle transmission energy consumption for this cycle \\
\hline 17 & idle_transmit & \\
\hline 18 & idle_listen & idergy consumption for this cycle \\
\hline
\end{tabular}

In Figure 6, the depicted Mote output window displays the captured "powertrace" information every $2 \mathrm{~s}$ and also the messages sent and received by each mote (printouts/printf messages from each mote).

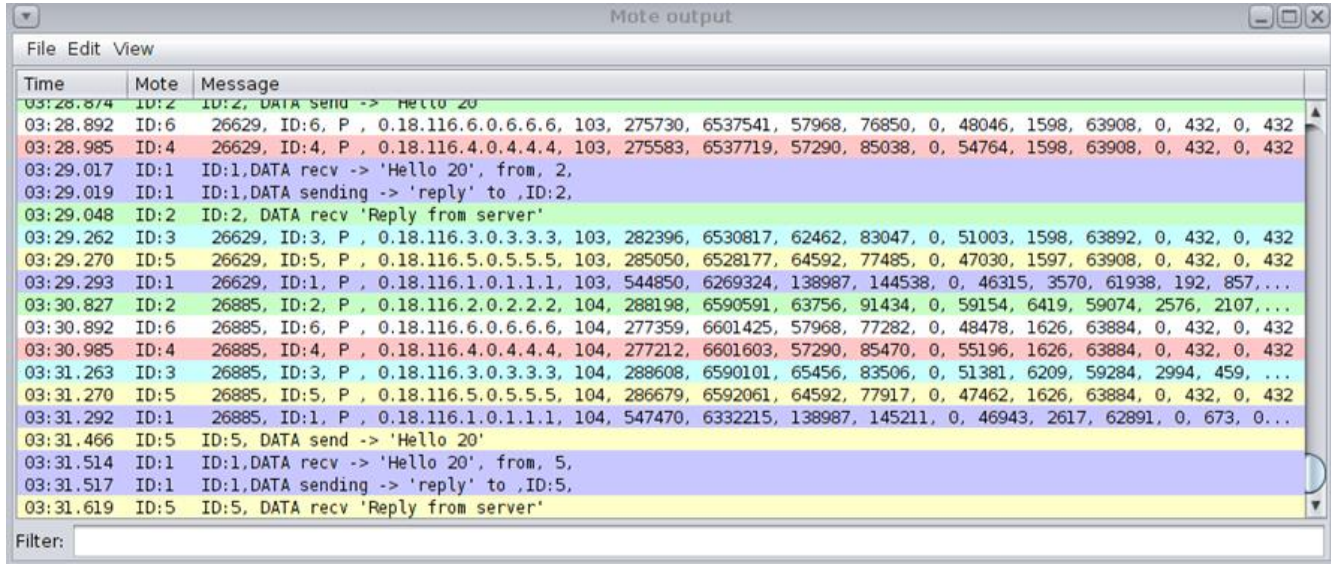

Figure 6. Cooja Simulator-Mote output window. 
Furthermore, the Simulation script editor, shown in Figure 7, is a Cooja tool used to display messages and set a timer on the simulation. As shown in Figure 7, the upper part of the Simulation script editor was used to create scripts and the lower part to show the captured "powertrace" information and the printouts (i.e., printf messages) from the motes until the timeout occurs. In our implementation, we considered the simulation duration to be $60 \mathrm{~min}$ and thus, the timeout was set at 3,600,000 ms. When the timeout occurred, the simulation stopped, and all the captured information and prints were stored in the log file named "COOJA.testlog".

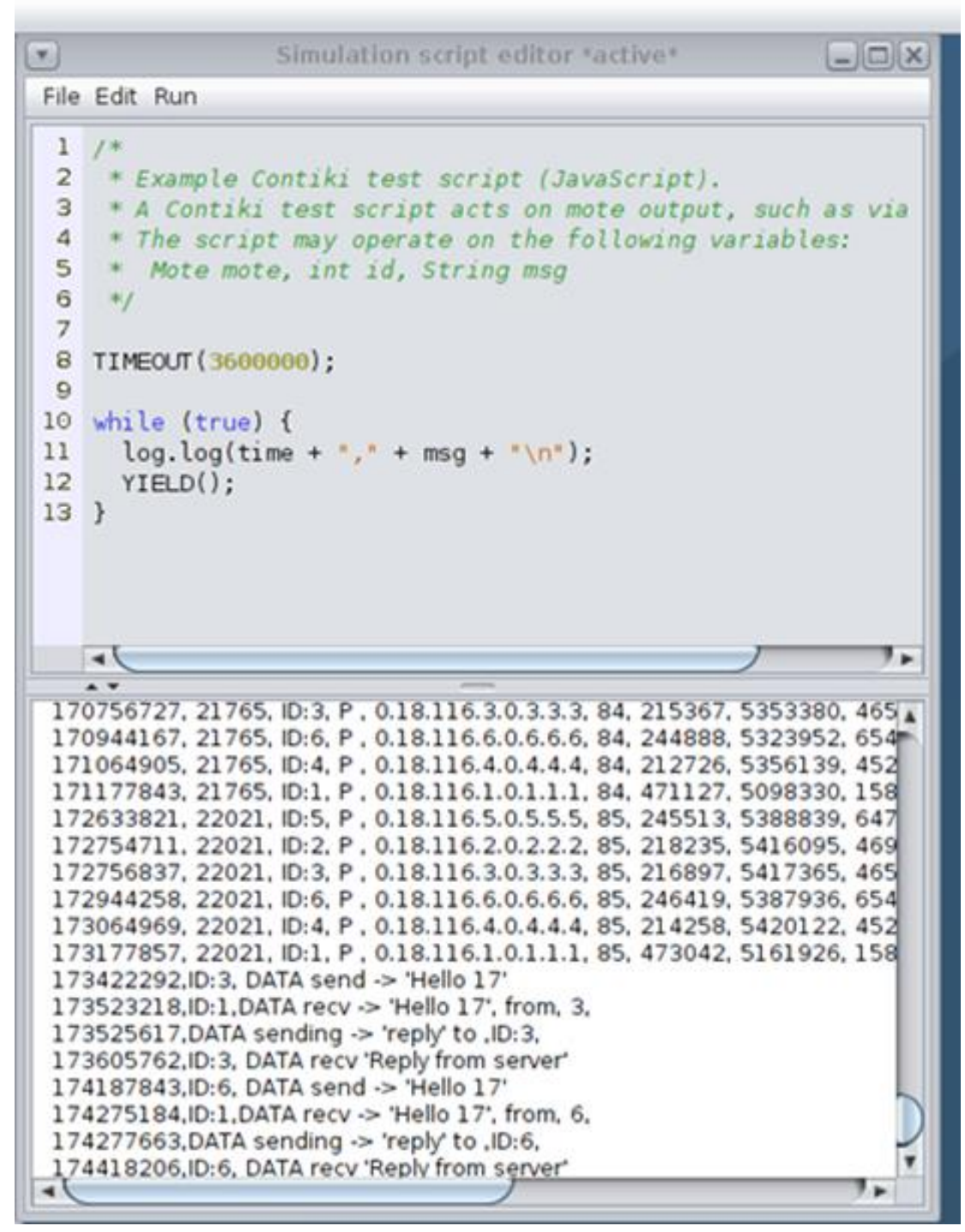

Figure 7. Simulation script editor.

Having collected all the captured raw information from the "powertrace" plugin in the "COOJA.testlog" file, the challenging task was to extract this information from the "COOJA.testlog" file to a csv file that would be the "powertrace" dataset of the simulated benign IIoT network scenario including records about the energy consumption of the motes. To address this challenge, we developed the "IoT_Simul.sh" bash file in order to extract all the required "powertrace" information from the "COOJA.testlog" file to the "pwrtrace.csv" file. An extract of the "IoT_Simul.sh" bash file is shown in Figure 8. 


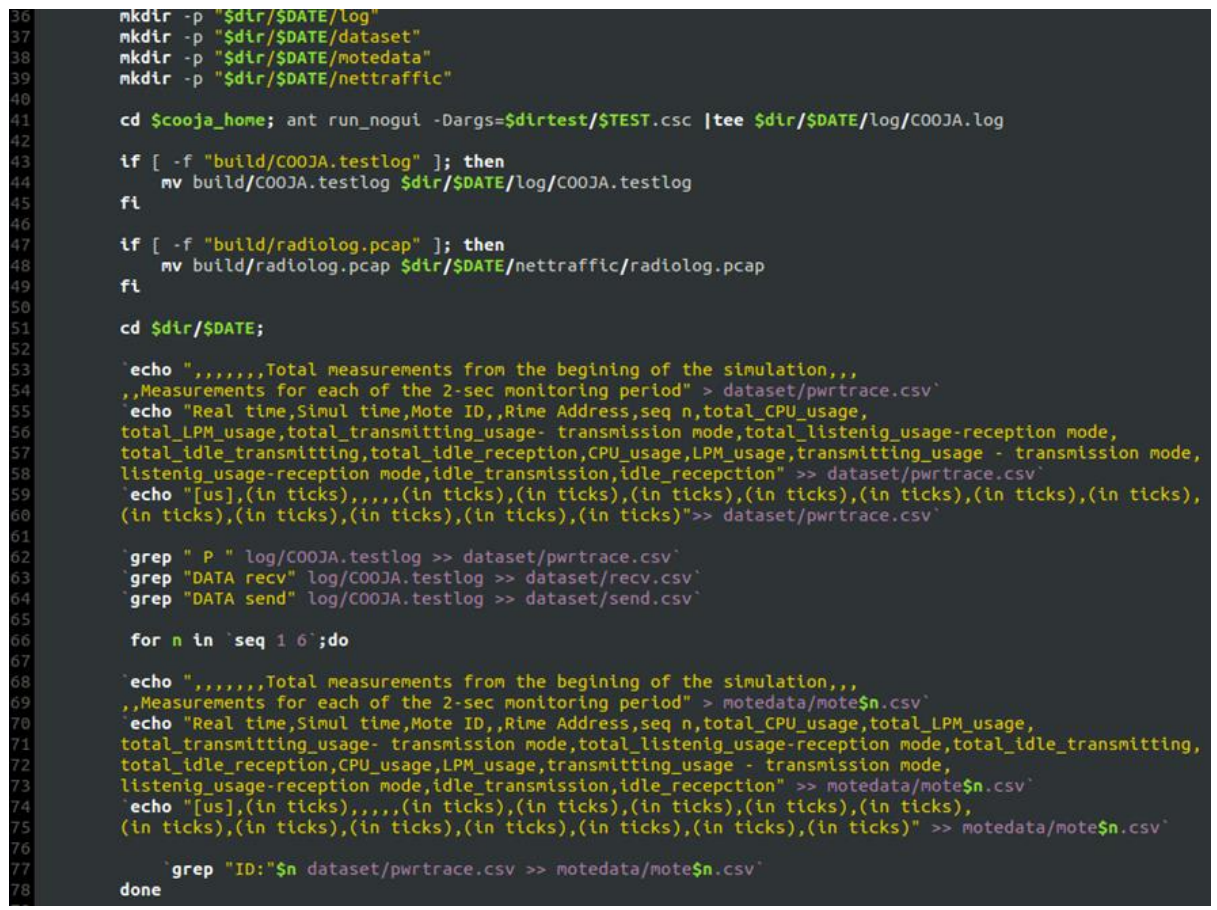

Figure 8. Extract of the "IoT_Simul.sh" bash file.

Initially, the "IoT_Simul.sh" file created the root folder which was named with the simulation date and time (i.e., "2020-11-19-17-45-22" folder), as shown below in the left part of Figure 9. Afterwards, the bash file created the "log" folder, inside the "2020-11-1917-45-22" folder, where the "COOJA.testlog" file was copied from the " .. / cooja/build" folder located in the Cooja Simulator environment.

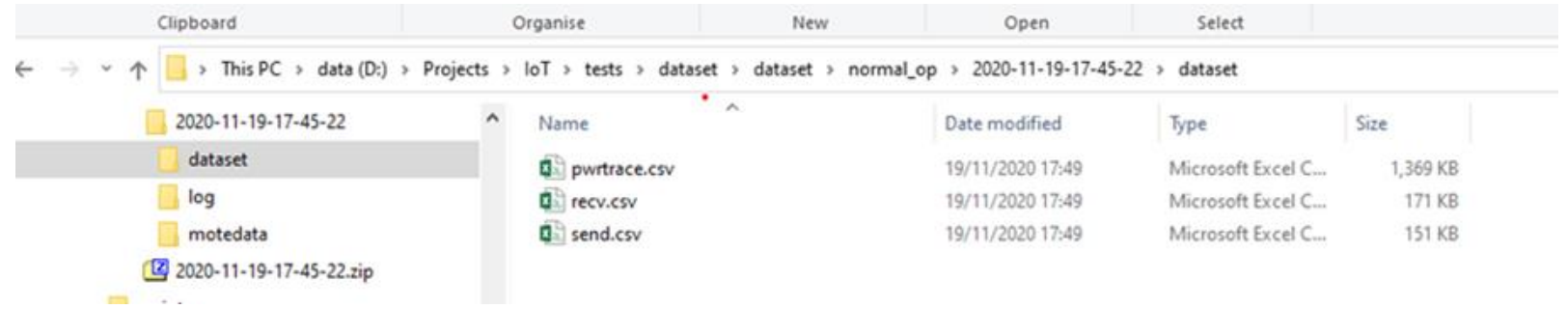

Figure 9. Location of the generated "pwrtrace.csv", "recv.csv", and "send.csv" files by the "IoT_Simul.sh" file.

In addition, in the "IoT_Simul.sh" file, we used the Linux tool "grep" in order to extract the required "powertrace" information by selecting the label " $\mathrm{P}$ " in each powertrace row (i.e., grep "P" $\log /$ COOJA.testlog >> dataset/pwrtrace.csv) from the "COOJA.testlog" file and save it in the "pwrtrace.csv" file in the "dataset" folder that was created by the batch file inside the "2020-11-19-17-45-22" folder, as shown in the left part of Figure 9. In the "dataset" folder, apart from the "pwrtrace.csv" file, the "IoT_Simul.sh" file generated two more files, based on the information included in the "COOJA.testlog" file, as shown in Figure 9; the "recv.csv" file and the "send.csv" file that include the "received" and "sent"messages printed by the motes, respectively.

Finally, the "IoT_Simul.sh" file extracted the information related to each mote, from the "pwrtrace.csv" file, and generated one csv file for each mote with the corresponding information from the "pwrtrace.csv" file. The generated $6 \mathrm{csv}$ files (i.e., mote1.csv, mote2.csv, mote3.csv, mote4.csv, mote5.csv, mote6.csv) were stored in the "motedata" folder. The "motedata" folder was also created by the "IoT_Simul.sh" file inside the "2020-11-19-17-4522 " folder. 
An overview of the above mentioned process followed to extract the required information from the "COOJA.testlog" file to the "pwrtrace.csv", "recv.csv", and "send.csv", "mote1.csv", "mote2.csv", "mote3.csv", "mote4.csv", "mote5.csv", and "mote6.csv" files are depicted in the Figure 10.

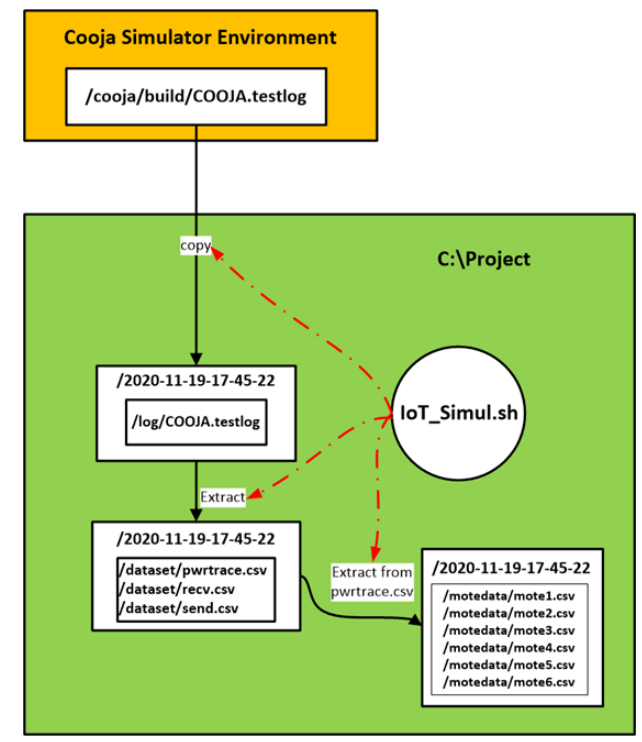

Figure 10. An overview of the process followed by the "IoT_Simul.sh" file to extract all the required "powertrace" information from the "COOJA.testlog" file.

\subsubsection{Benign "Powertrace" Datasets—Results}

Benign "pwrtrace.csv": The generated benign "pwrtrace.csv" file consists of 10,794 records and its first 38 records (i.e., $1-38)$ and its last 38 records $(10,757-10,794)$ are depicted in Figures 11 and 12, respectively.

\begin{tabular}{|c|c|c|c|c|c|c|c|c|c|c|c|c|c|c|c|c|c|c|}
\hline \multirow[t]{2}{*}{ B } & o & E & F & $\sigma$ & H & 1 & $\mathrm{x}$ & $i$ & M & $\mathrm{N}$ & 0 & p & $\mathbf{R}$ & s & $\mathbf{T}$ & u & $\mathrm{v}$ & w \\
\hline & & & & & & & \multicolumn{6}{|c|}{ Total measurements from the begining of the simulation } & \multicolumn{6}{|c|}{ Measurements every 2 seconds (monitoring period) } \\
\hline No & $\begin{array}{l}\text { Real time } \\
\text { [us] }\end{array}$ & $\begin{array}{l}\text { dock_time } \\
\text { (in ticks) }\end{array}$ & 10 & p & rimeaddr & seqno & $\begin{array}{l}\text { all_cpu } \\
\text { (in ticks) }\end{array}$ & $\begin{array}{l}\text { all_.lpm } \\
\text { (in ticks) }\end{array}$ & $\begin{array}{l}\text { all_tranumit } \\
\text { (in tids) }\end{array}$ & $\begin{array}{l}\text { all_listen } \\
\text { (in ticks) }\end{array}$ & $\begin{array}{c}\text { all_idele_transmit } \\
\text { (in tikks) }\end{array}$ & $\begin{array}{c}\text { all_Jidle_listen } \\
\text { (in ticks) }\end{array}$ & $\begin{array}{c}\text { cpu } \\
\text { (in tieds) }\end{array}$ & $\begin{array}{c}\text { lpm } \\
\text { (in tikks) }\end{array}$ & $\begin{array}{l}\text { transmit } \\
\text { (in ticks) }\end{array}$ & $\begin{array}{l}\text { luten } \\
\text { (in ticks) }\end{array}$ & $\begin{array}{c}\text { Idle_tranamit } \\
\text { (in ticks) }\end{array}$ & $\begin{array}{l}\text { Ialle_listen } \\
\text { (in ticks) }\end{array}$ \\
\hline 1 & 2587177 & 261 & 10.6 & p & 0.18 .116 .60 .6 .6 .6 & 0 & 6737 & 59719 & 2588 & 442 & 0 & 364 & 6737 & 59719 & 2588 & 442 & 0 & 364 \\
\hline 2 & 2816245 & 261 & ID:3 & $p$ & 0.18 .116 .30 .3 .3 & 0 & 2184 & 64270 & 0 & 390 & 0 & 390 & 2184 & 64270 & 0 & 300 & 0 & 390 \\
\hline 3 & 2907083 & 261 & ID:1 & p & 0.18 .116 .10 .1 .1 .1 & 0 & 2827 & 63628 & 0 & 2003 & 0 & 744 & 2827 & 63628 & 0 & 1003 & 0 & 744 \\
\hline 4 & 3163478 & 261 & $10: 4$ & $P$ & 0.18 .116 .40 .4 .44 & 0 & 2184 & 64270 & 0 & 390 & 0 & 390 & 2184 & 64270 & 0 & 390 & 0 & 390 \\
\hline 5 & 3183394 & 261 & 10.5 & $p$ & 0.18116 .50 .55 .5 & 0 & 6737 & 59719 & 2588 & 442 & 0 & 364 & 6737 & 59719 & 2588 & 442 & 0 & 364 \\
\hline 6 & 3305496 & 261 & $10 \cdot 2$ & $p$ & 0.18 .116 .20 .22 .2 & 0 & 6737 & 59719 & 2588 & 442 & 0 & 364 & 6737 & 59719 & 2588 & 442 & 0 & 364 \\
\hline 7 & 4586462 & 517 & 10.6 & $p$ & 0.18 .116 .60 .6 .6 .6 & 1 & 7899 & 124068 & 2588 & 858 & 0 & 780 & 1159 & 64349 & 0 & 416 & 0 & 416 \\
\hline 8 & 4821159 & 517 & 103 & $p$ & 0.18 .116 .30 .3 .3 .3 & 1 & 3569 & 128521 & 0 & 1094 & 0 & 1043 & 1382 & 64251 & 0 & 704 & 0 & 653 \\
\hline 9 & 4909515 & 517 & $10=1$ & $p$ & 0.18 .116 .10 .1 .1 .1 & 1 & 8583 & 123383 & 2980 & 1472 & 0 & 1134 & 5753 & 59735 & 2980 & 469 & 0 & 390 \\
\hline 10 & 5164736 & 517 & $10: 4$ & $p$ & 0.18116 .40 .4 .44 & 1 & 3625 & 128346 & 0 & 1105 & 0 & 1056 & 1438 & 64076 & 0 & 715 & 0 & 666 \\
\hline 11 & 5183527 & 517 & 105 & $p$ & 0.18 .116 .50 .5 .5 .5 & 1 & 8248 & 123723 & 2588 & 1158 & 0 & 1030 & 1508 & 64004 & 0 & 716 & 0 & 606 \\
\hline 12 & 5305285 & 517 & $10-2$ & $p$ & 0.18 .116 .20 .2 .2 .2 & 1 & 8250 & 123721 & 2588 & 1133 & 0 & 754 & 1510 & 64002 & 0 & 691 & 0 & 390 \\
\hline 13 & 6587344 & 773 & 10.6 & $p$ & 0.18116 .60 .6 .6 .6 & 2 & 9564 & 187918 & 2588 & 1525 & 0 & 1170 & 1662 & 63850 & 0 & 667 & 0 & 390 \\
\hline 14 & 6817450 & $m 3$ & $10: 3$ & $p$ & 0.18116 .30 .3 .3 .3 & 2 & 4957 & 192526 & 0 & 1510 & 0 & 1459 & 1385 & 64005 & 0 & 416 & 0 & 416 \\
\hline 15 & 6909795 & $m$ & 101 & $p$ & 0.18116 .10 .1 .1 .1 & 2 & 10071 & 187437 & 2980 & 2173 & 0 & 1537 & 1486 & 64054 & 0 & 701 & 0 & 403 \\
\hline 16 & 7164685 & $m_{3}$ & 10.4 & $p$ & 0.18 .116 .40 .4 .4 .4 & 2 & 5014 & 192466 & 0 & 1521 & 0 & 1472 & 1386 & 64120 & 0 & 416 & 0 & 416 \\
\hline 17 & 7184917 & $m$ & 10.5 & P & 0.18116 .50 .55 .5 & 2 & 14271 & 183210 & 5572 & 1630 & 0 & 1420 & 6020 & 59487 & 2984 & 472 & 0 & 390 \\
\hline 18 & 7307013 & $m 3$ & 1022 & $p$ & 0.18 .116 .20 .2 .2 .2 & 2 & 14255 & 183225 & 5565 & 1601 & 0 & 1144 & 6002 & 59504 & 29077 & 468 & 0 & 390 \\
\hline 19 & 8588987 & 1029 & 106 & $P$ & 0.18 .116 .60 .6 .6 .6 & 3 & 15557 & 247436 & 5573 & 2968 & 0 & 1534 & 5990 & 59518 & 2085 & 443 & 0 & 364 \\
\hline 20 & 8820944 & 1029 & $10: 3$ & $P$ & 0.18 .116 .30 .3 .3 .3 & 3 & 18623 & 244367 & 79042 & 4101 & 0 & 1823 & 13663 & 51841 & 7942 & $259 !$ & 0 & 364 \\
\hline 21 & 8509413 & 1029 & $10: 1$ & $p$ & 0.18 .116 .10 .11 .1 & 3 & 13115 & 249855 & 2980 & 4355 & 0 & 2453 & 3041 & 62418 & 0 & 2182 & 0 & 916 \\
\hline 22 & 9168183 & 1029 & 104 & $p$ & 0.18 .116 .4 .0 .4 .4 .4 & 3 & 18227 & 244754 & 7542 & 3922 & 0 & 1810 & 13210 & 52283 & 7542 & 2401 & 0 & 333 \\
\hline 23 & 9185894 & 1029 & IDS & $p$ & 0.18 .116 .50 .5 .5 .5 & 3 & 23353 & 239636 & 10452 & 4102 & 0 & 1784 & 9079 & 56426 & 4880 & 2472 & 0 & 364 \\
\hline 24 & 9306227 & 1029 & $10: 2$ & $p$ & 0.18 .116 .20 .22 .2 & 3 & 15749 & 247241 & 5565 & 2017 & 0 & 1560 & 1491 & 64016 & 0 & 416 & 0 & 416 \\
\hline 25 & 10656477 & 1293 & 10.6 & $p$ & 0.18 .116 .60 .66 .6 & 4 & 19726 & 310973 & 7091 & 3102 & 0 & 1950 & 4166 & 63537 & 1518 & 1134 & 0 & 416 \\
\hline 26 & 10819122 & 1285 & $1 D=3$ & $p$ & 0.18 .116 .30 .3 .3 .3 & 4 & 20093 & 308390 & 7942 & 4517 & 0 & 2239 & 1468 & 64023 & 0 & 416 & 0 & 416 \\
\hline 27 & 10909061 & 1285 & $1 D=1$ & $p$ & 0.18 .116 .10 .1 .1 .1 & 4 & 15371 & 313112 & 2980 & 5170 & 0 & 2817 & 2253 & 63257 & 0 & 815 & 0 & 364 \\
\hline 28 & 11166334 & 1285 & $10 \cdot 4$ & $p$ & 0.18 .116 .40 .4 .4 .4 & 4 & 19655 & 308818 & 7542 & 4338 & 0 & 2226 & 1426 & 64064 & 0 & 416 & 0 & 416 \\
\hline 29 & 11184417 & 1285 & 10.5 & $p$ & 0.18116 .50 .55 .5 & 4 & 24780 & 303701 & 10452 & 4518 & 0 & 2200 & 1425 & 64065 & 0 & 416 & 0 & 416 \\
\hline 30 & 11306888 & 1285 & 102 & $p$ & 0.18 .116 .20 .22 .2 & 4 & 17828 & 310652 & 5726 & 2610 & 0 & 1976 & 2076 & 63411 & 161 & 593 & 0 & 416 \\
\hline 31 & 12588011 & 1541 & 10.6 & $p$ & 0.18116 .60 .6 .6 .6 & 5 & 21486 & 372532 & 7091 & 3990 & 0 & 2543 & 1757 & 61559 & 0 & 883 & 0 & 593 \\
\hline 32 & 12819256 & 1 SA1 & $10 \cdot 3$ & $p$ & 0.18 .116 .30 .3 .3 .3 & 5 & 21848 & 372149 & 7942 & 5306 & 0 & 2806 & 1753 & 63739 & 0 & 789 & 0 & 567 \\
\hline 33 & 12910930 & 1541 & $10: 1$ & $p$ & 0.18116 .10 .1 .1 .1 & 5 & 26285 & 367714 & 8402 & 7027 & 0 & 3142 & 10911 & 54602 & 5422 & 1857 & 0 & 325 \\
\hline 34 & 13168185 & 1541 & $10: 4$ & $p$ & 0.18 .16 .40 .4 .4 .4 & 5 & 26062 & 367921 & 10016 & 6692 & 0 & 2780 & 6404 & 59103 & 2474 & 2354 & 0 & 554 \\
\hline 35 & 13184502 & 1541 & 105 & p & 0.18 .116 .50 .5 .5 .5 & 5 & 26537 & 367458 & 10452 & 5169 & 0 & 2590 & 1754 & 63757 & 0 & 651 & 0 & 390 \\
\hline 36 & 13306133 & 1541 & 10.2 & $P$ & $0.18 \cdot 116.20 .2 .2 .2$ & 5 & 19600 & 374395 & 5726 & 3329 & 0 & 2379 & 1769 & 63743 & 0 & 719 & 0 & 403 \\
\hline 37 & 14589285 & 1797 & 10.6 & p & 0.18116 .60 .6 .6 .6 & 6 & 27550 & 431978 & 10073 & 4458 & 0 & 2933 & 6061 & 59445 & 2982 & 468 & 0 & 390 \\
\hline 38 & 14819832 & 1797 & 103 & p & 0.18 .116 .30 .3 .3 .3 & 6 & 24234 & 435261 & 8052 & 6065 & 0 & 3209 & 2383 & 63112 & 110 & 760 & 0 & 403 \\
\hline
\end{tabular}

Figure 11. Benign "pwrtrace.csv"-1 to 38 records. 


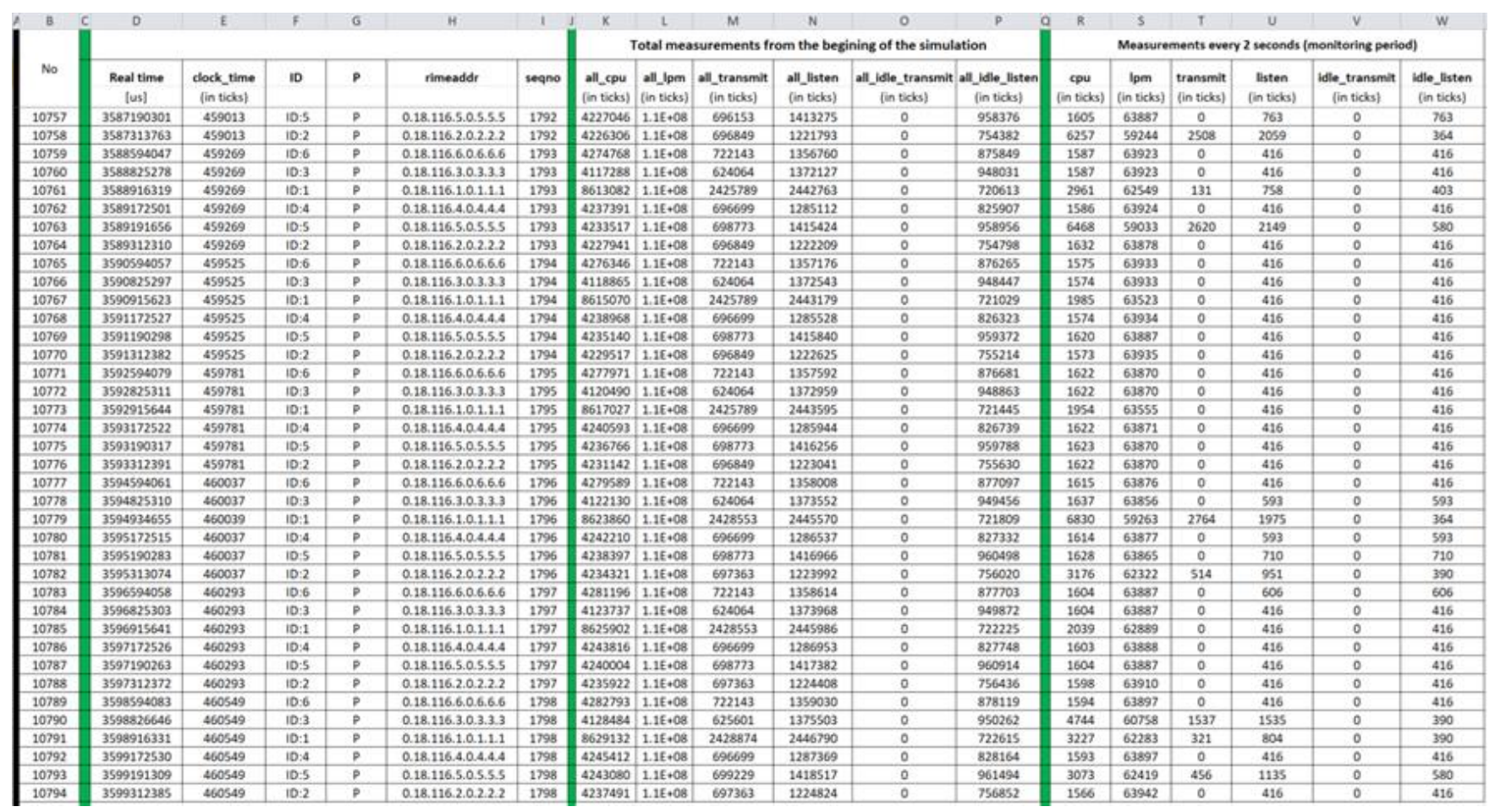

Figure 12. Benign "pwrtrace.csv" $-10,757$ to 10,794 records.

Benign "recv.csv": The generated benign "recv.csv" file consists of 3586 records and its first 25 records (i.e., 1-25) are depicted below in Figure 13.

\begin{tabular}{|c|c|c|c|c|c|}
\hline B & c & D & E & $\mathrm{F}$ & G \\
\hline No & Real time [us] & $\begin{array}{c}\text { ID } \\
\text { (Receiver) }\end{array}$ & Received Message & & $\begin{array}{c}\text { ID } \\
\text { (Sender) }\end{array}$ \\
\hline 1 & 11635659 & ID:1 & DATA recv $>>$ 'Hello 1 ' & from & 4 \\
\hline 2 & 11768650 & ID:4 & DATA recv 'Reply from server' & & \\
\hline 3 & 14510081 & ID:1 & DATA recv $>>$ 'Hello 1' & from & 3 \\
\hline 4 & 14545397 & ID:3 & DATA recv 'Reply from server' & & \\
\hline 5 & 16259239 & ID:1 & DATA recv $>>$ 'Hello 1' & from & 2 \\
\hline 6 & 16531142 & ID:2 & DATA recv 'Reply from server' & & \\
\hline 7 & 18258289 & ID:1 & DATA recv $>>$ 'Hello 1' & from & 5 \\
\hline 8 & 18283595 & ID:5 & DATA recv 'Reply from server' & & \\
\hline 9 & 19884821 & ID:1 & DATA recv $>>$ 'Hello 1' & from & 6 \\
\hline 10 & 19937444 & ID:6 & DATA recv 'Reply from server' & & \\
\hline 11 & 23761798 & ID:1 & DATA recv -> 'Hello 2' & from & 4 \\
\hline 12 & 23891542 & ID:4 & DATA recv 'Reply from server' & & \\
\hline 13 & 24385405 & ID:1 & DATA recv $>>$ 'Hello 2' & from & 6 \\
\hline 14 & 24437891 & ID:6 & DATA recv 'Reply from server' & & \\
\hline 15 & 28008873 & ID:1 & DATA recv $>>$ 'Hello 2' & from & 5 \\
\hline 16 & 28034048 & ID:5 & DATA recv 'Reply from server' & & \\
\hline 17 & 29634363 & ID:1 & DATA recv -> 'Hello 2' & from & 3 \\
\hline 18 & 29669812 & ID:3 & DATA recv 'Reply from server' & & \\
\hline 19 & 30134905 & ID:1 & DATA recv -> 'Hello 2' & from & 2 \\
\hline 20 & 30281255 & ID:2 & DATA recv 'Reply from server' & & \\
\hline 21 & 31258819 & ID:1 & DATA recv -> 'Hello 3' & from & 3 \\
\hline 22 & 31294158 & ID:3 & DATA recv 'Reply from server' & & \\
\hline 23 & 35260414 & ID:1 & DATA recv -> 'Hello 3' & from & 6 \\
\hline 24 & 35312814 & ID:6 & DATA recv 'Reply from server' & & \\
\hline 25 & 38883782 & ID:1 & DATA recv $>>$ 'Hello 3' & from & 2 \\
\hline
\end{tabular}

Figure 13. Benign "recv.csv" -1 to 25 records.

\subsection{Benign Network Traffic Dataset Generation}

\subsubsection{Benign Network Traffic Dataset Generation}

The generated network traffic dataset constitutes the dataset of the simulated benign IIoT network scenario that includes records consisting of IIoT network traffic features such as source/destination IPv6 address, packet size, and communication protocol. The Cooja simulator provides the "Radio messages" tool that allowed the collection of data related 
to the corresponding network traffic features. In Figure 14, the "Radio messages" output window is depicted along with the three configuration options that are provided by the "Radio messages" tool:

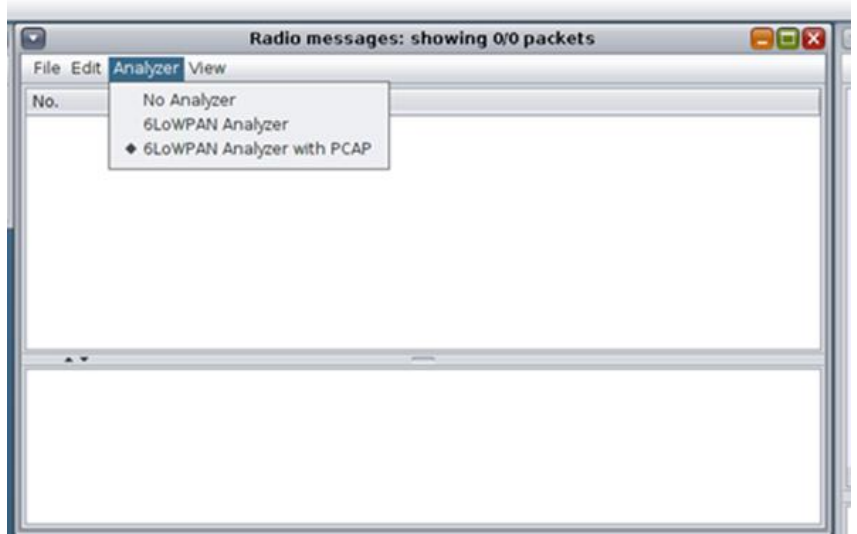

Figure 14. "Radio messages" tool—output window.

The "6LoWPAN Analyzer with PCAP" option was selected and the "Radio messages" tool saved the captured network traffic data from the simulated IIoT network into a pcap file whose file-naming format was as follows: "radiolog-" + System.currentTimeMillis() + "pcap".

During the simulation, the network traffic information about the transmitted data was also being shown in the top part of the "Radio messages" output window as depicted in the top part of Figure 15. When the simulation stopped, the generated pcap file was saved as "radiolog-1605811324302.pcap" within the "... / cooja/build" folder.

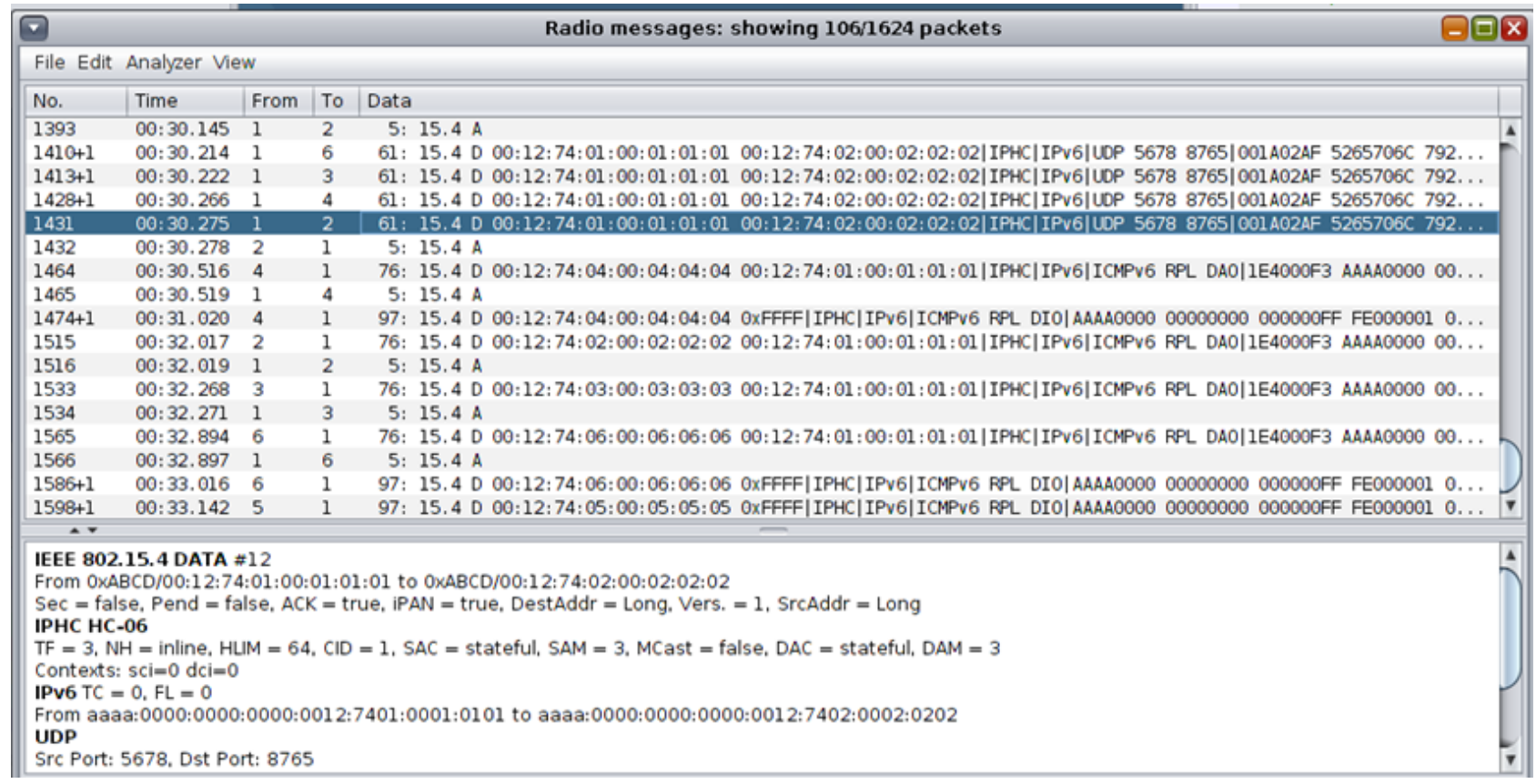

Figure 15. Network traffic information from the benign scenario in the "Radio messages" output window.

Having now saved all the captured raw network traffic information, through the "Radio messages" tool, into a pcap file, the challenging task was to extract this information from the pcap file to a csv file that would be the network traffic dataset of the simulated benign IIoT network scenario. This challenge was addressed by utilising the "IoT_Simul.sh" file that was also used in the "powertrace" dataset generation process, as described in Section 4.1, and the well-known network protocol analyser Wireshark [28]. 
In particular, the first step was the use of the "IoT_Simul.sh" file in order to copy the "radiolog-1605811324302.pcap" file from the "... / cooja/build" folder located in the Cooja Simulator environment to the "nettraffic" folder that was created by the "IoT_Simul.sh" file inside the root folder "2020-11-19-17-45-22" that was also created by the "IoT_Simul.sh" during the "powertrace" dataset generation process. The "nettraffic" folder inside the root folder "2020-11-19-17-45-22" and the copy of the "radiolog-1605811324302.pcap" file in the "nettraffic" folder is shown in Figure 16.

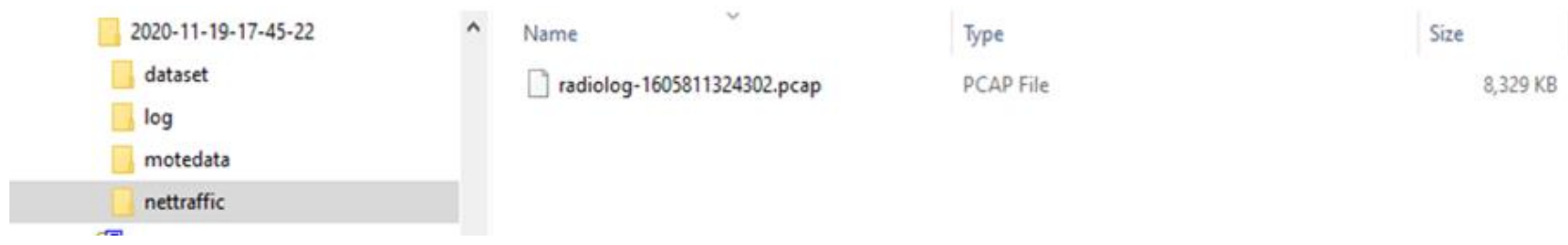

Figure 16. The "nettraffic" folder inside the root folder "2020-11-19-17-45-22" and the copy of the "radiolog1605811324302.pcap" file.

After having the copy of the "radiolog-1605811324302.pcap" file in the "nettraffic" folder, the next step was the extraction of the stored network traffic information from the "radiolog-1605811324302.pcap" file to the "radiolog.csv" file. This was achieved through Wireshark as Wireshark allows opening a pcap file and exporting data to a csv file. In Figure 17, the upper panel of the Wireshark window shows the seventeen first packets included in the "radiolog-1605811324302.pcap" file that was opened via Wireshark. The middle panel shows the protocol details of the 10th packet selected in the upper panel and the bottom panel presents the protocol details of the selected 10th packet in both HEX and ASCII format.

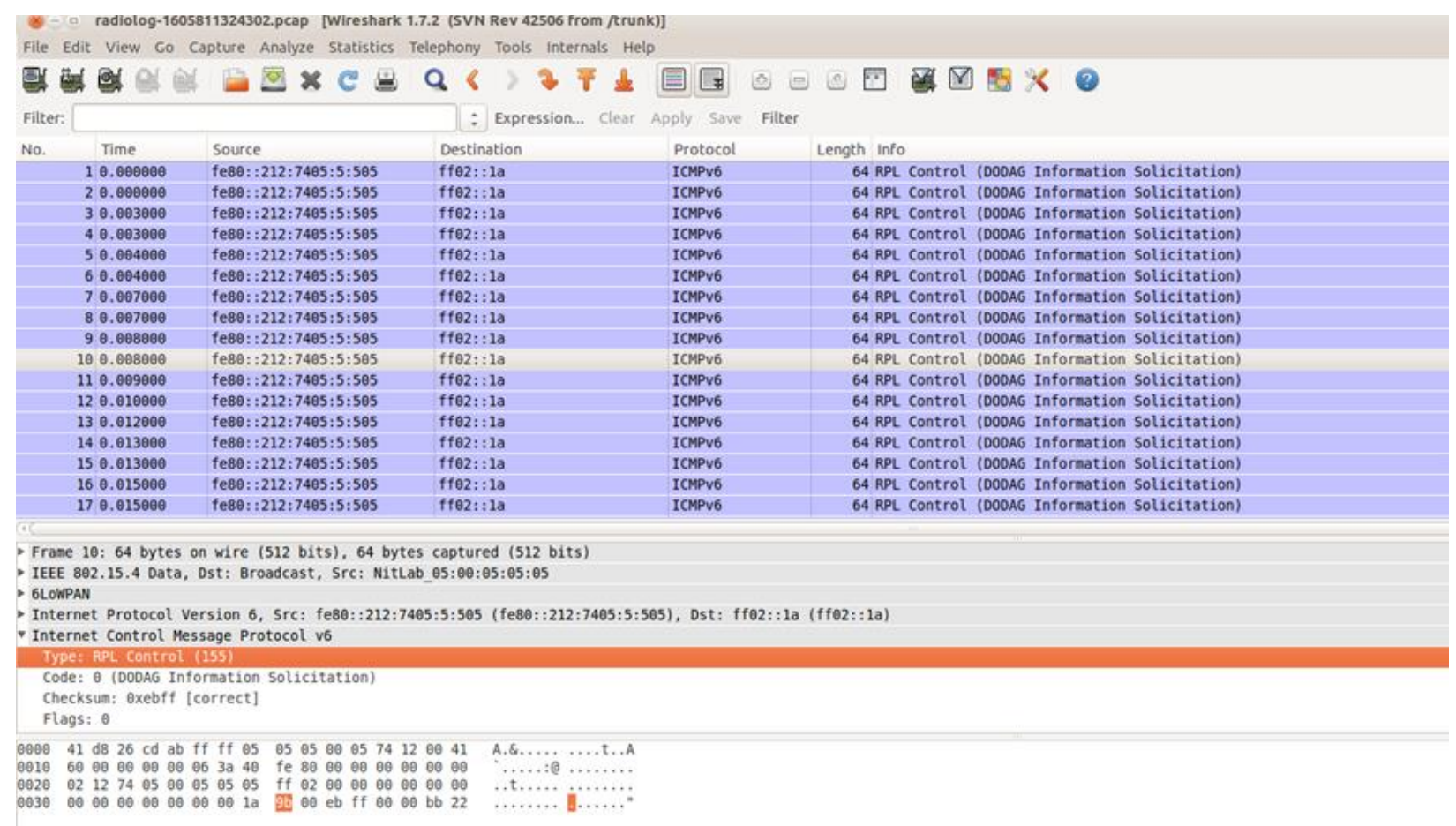

Figure 17. The first seventeenth packets in the "radiolog-1605811324302.pcap" file. 
The data from the "radiolog-1605811324302.pcap" file were exported and saved, through Wireshark, into the "radiolog.csv" file in the "nettraffic" folder in the project environment, as shown in Figure 18. Furthermore, it is worthwhile to mention that we also used Wireshark to filter the "radiolog-1605811324302.pcap" file based on the ICMPv6 protocol and the UDP protocol and then exported and saved the filtered results, through Wireshark, in the "radiologICMPv6.csv" file and the "radiologUDP.csv" file, respectively, in the "nettraffic" folder in the project environment, as shown in Figure 19. The radiologICMPv6.csv" file and the "radiologUDP.csv" file facilitated the analysis of the capture traffic as shown in Section 6.

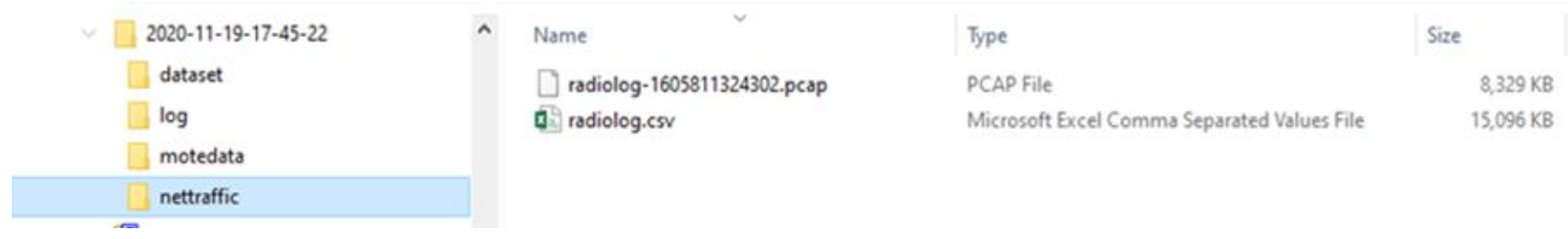

Figure 18. The "radiolog.csv" file in the "nettraffic" folder in the project environment.

\begin{tabular}{|c|c|c|c|c|}
\hline 2020-11-19-17-45-22 & $\hat{\imath}$ & Name & Type & Size \\
\hline$\square$ dataset & & D radiolog-1605811324302.pcap & PCAP File & $8,329 \mathrm{~KB}$ \\
\hline$\square \log$ & & (4) radiolog.csv & Microsoft Excel Comma Separated Values File & $15,096 \mathrm{~KB}$ \\
\hline 1 motedata & & 4. radiologUDP.csV & Microsoft Excel Comma Separated Values File & $13,938 \mathrm{~KB}$ \\
\hline nettraffic & & 4. radiologICMPv6.csv & Microsoft Excel Comma Separated Values File & $929 \mathrm{~KB}$ \\
\hline
\end{tabular}

Figure 19. The "radiologICMPv6.csv" file and the "radiologUDP.csv" file in the "nettraffic" folder in the project environment.

Finally, an overview of the above mentioned process followed to extract the required information from the "radiolog-1605811324302.pcap" file to the "radiolog.csv", "radiologICMPv6.csv" and "radiologUDP.csv" files is depicted in Figure 20.

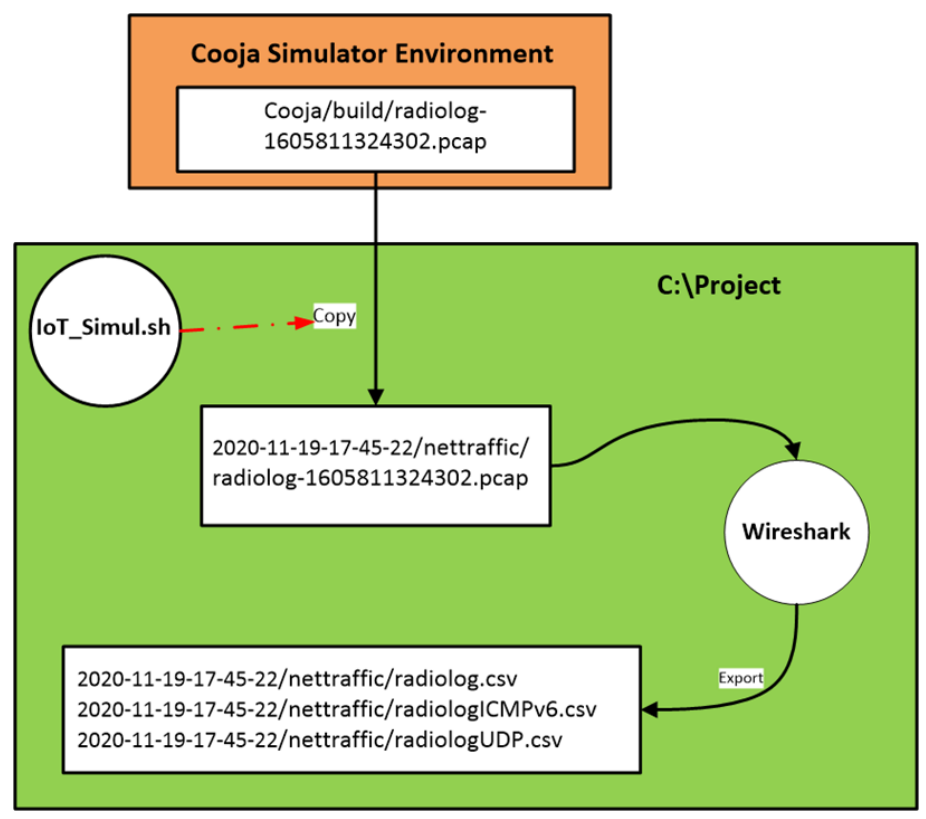

Figure 20. An overview of the process followed to extract all the required network traffic information from the "radiolog-1605811324302.pcap" file. 


\subsubsection{Benign Network Traffic Datasets-Results}

"radiolog.csv": The generated benign "radiolog.csv" file consists of 116,463 records and its first 40 records (i.e., 1-40) are depicted below in Figure 21.

\begin{tabular}{|c|c|c|c|c|c|c|}
\hline B & c & D & E & $\mathbf{F}$ & G & H \\
\hline No & Time (sec) & Source Address (IPv6) & Destination Address (IPv6) & Protocol & $\begin{array}{l}\text { Length } \\
\text { (bytes) }\end{array}$ & Info \\
\hline 1 & 0 & fe80::212:7405:5:505 & fffo2::1a & ICMPV6 & 64 & RPL Control (DODAG Information Solicitation) \\
\hline 2 & 0 & fe80::212:7405:5:505 & ff02::1a & ICMPv6 & 64 & RPL Control (DODAG Information Solicitation) \\
\hline 3 & 0.003 & fe80::212:7405:5:505 & ffo2::1a & ICMPV6 & 64 & RPL Control (DODAG Information Solicitation) \\
\hline 4 & 0.003 & fe80::212:7405:5:505 & fffo2::1a & ICMPV6 & 64 & RPL Control (DODAG Information Solicitation) \\
\hline 5 & 0.004 & fe80::212:7405:5:505 & ffo2::1a & ICMPV6 & 64 & RPL Control (DODAG Information Solicitation) \\
\hline 6 & 0.004 & fe80::212:7405:5:505 & ffo2::1a & ICMPV6 & 64 & RPL Control (DODAG Information Solicitation) \\
\hline 7 & 0.007 & fe80::212:7405:5:505 & ffo2::1a & ICMPV6 & 64 & RPL Control (DODAG Information Solicitation) \\
\hline 8 & 0.007 & fe80::212:7405:5:505 & fffo2::1a & ICMPV6 & 64 & RPL Control (DODAG Information Solicitation) \\
\hline 9 & 0.008 & fe80::212:7405:5:505 & ffo2::1a & ICMPV6 & 64 & RPL Control (DODAG Information Solicitation) \\
\hline 10 & 0.008 & fe80::212:7405:5:505 & ffo2::1a & ICMPV6 & 64 & RPL Control (DODAG Information Solicitation) \\
\hline 11 & 0.009 & fe80::212:7405:5:505 & ffo2::1a & ICMPv6 & 64 & RPL Control (DODAG Information Solicitation) \\
\hline 12 & 0.01 & fe80::212:7405:5:505 & ffo2::1a & ICMPV6 & 64 & RPL Control (DODAG Information Solicitation) \\
\hline 13 & 0.012 & fe80::212:7405:5:505 & ffo2::1a & ICMPV6 & 64 & RPL Control (DODAG Information Solicitation) \\
\hline 14 & 0.013 & fe80::212:7405:5:505 & fffo2::1a & ICMPv6 & 64 & RPL Control (DODAG Information Solicitation) \\
\hline 15 & 0.013 & fe80::212:7405:5:505 & ff02::1a & ICMPV6 & 64 & RPL Control (DODAG Information Solicitation) \\
\hline 16 & 0.015 & fe $80:: 212: 7405: 5: 505$ & ff02::1a & ICMPV6 & 64 & RPL Control (DODAG Information Solicitation) \\
\hline 17 & 0.015 & fe $80:: 212: 7405: 5: 505$ & ffo2::1a & ICMPV6 & 64 & RPL Control (DODAG Information Solicitation) \\
\hline 18 & 0.019 & fe80::212:7405:5:505 & ffo2::1a & ICMPV6 & 64 & RPL Control (DODAG Information Solicitation) \\
\hline 19 & 0.02 & fe80::212:7405:5:505 & ffo2::1a & ICMPV6 & 64 & RPL Control (DODAG Information Solicitation) \\
\hline 20 & 0.021 & fe80::212:7405:5:505 & fffo2::1a & ICMPV6 & 64 & RPL Control (DODAG Information Solicitation) \\
\hline 21 & 0.021 & fe80::212:7405:5:505 & ff02::1a & ICMPV6 & 64 & RPL Control (DODAG Information Solicitation) \\
\hline 22 & 0.022 & fe80::212:7405:5:505 & ffo2::1a & ICMPV6 & 64 & RPL Control (DODAG Information Solicitation) \\
\hline 23 & 0.023 & fe80::212:7405:5:505 & ffo2::1a & ICMPV6 6 & 64 & RPL Control (DODAG Information Solicitation) \\
\hline 24 & 0.024 & fe80::212:7405:5:505 & ffo2::1a & ICMPV6 & 64 & RPL Control (DODAG Information Solicitation) \\
\hline 25 & 0.028 & fe $80:: 212: 7405: 5: 505$ & ff02::1a & ICMPV6 & 64 & RPL Control (DODAG Information Solicitation) \\
\hline 26 & 0.029 & fe80::212:7405:5:505 & ff02::1a & ICMPV6 & 64 & RPL Control (DODAG Information Solicitation) \\
\hline 27 & 0.029 & fe80::212:7405:5:505 & ffo2::1a & ICMPV6 & 64 & RPL Control (DODAG Information Solicitation) \\
\hline 28 & 0.029 & fe80::212:7405:5:505 & ffo2::1a & ICMPV6 & 64 & RPL Control (DODAG Information Solicitation) \\
\hline 29 & 0.03 & fe80::212:7405:5:505 & ffo2::1a & ICMPV6 & 64 & RPL Control (DODAG Information Solicitation) \\
\hline 30 & 0.031 & fe80::212:7405:5:505 & fffo2::1a & ICMPv6 & 64 & RPL Control (DODAG Information Solicitation) \\
\hline 31 & 0.031 & fe $80:: 212: 7405: 5: 505$ & ff02::1a & ICMPV6 & 64 & RPL Control (DODAG Information Solicitation) \\
\hline 32 & 0.039 & fe80::212:7405:5:505 & ffo2::1a & ICMPV6 & 64 & RPL Control (DODAG Information Solicitation) \\
\hline 33 & 0.039 & fe80::212:7405:5:505 & fffo2::1a & ICMPV6 & 64 & RPL Control (DODAG Information Solicitation) \\
\hline 34 & 0.04 & fe80::212:7405:5:505 & ffo2::1a & ICMPV6 & 64 & RPL Control (DODAG Information Solicitation) \\
\hline 35 & 0.04 & fe $80:: 212: 7405: 5: 505$ & ffo2::1a & ICMPV6 & 64 & RPL Control (DODAG Information Solicitation) \\
\hline 36 & 0.041 & fe80::212:7405:5:505 & ff02::1a & ICMPV6 & 64 & RPL Control (DODAG Information Solicitation) \\
\hline 37 & 0.041 & fe80::212:7405:5:505 & ffo2::1a & ICMPV6 & 64 & RPL Control (DODAG Information Solicitation) \\
\hline 38 & 0.042 & fe80::212:7405:5:505 & fffo2::1a & ICMPV6 & 64 & RPL Control (DODAG Information Solicitation) \\
\hline 39 & 0.24 & fe80::212:7406:6:606 & ffo2::1a & ICMPV6 & 64 & RPL Control (DODAG Information Solicitation) \\
\hline 40 & 0.241 & fe80::212:7406:6:606 & ffo2::1a & ICMPv6 & 64 & RPL Control (DODAG Information Solicitation) \\
\hline
\end{tabular}

Figure 21. Benign "radiolog.csv" -1 to 40 records.

"radiologICMPv6.csv": The generated benign "radiologICMPv6.csv" file consists of 7975 records and its last 28 records (i.e., 7948-7975) are depicted below in Figure 22.

\begin{tabular}{|c|c|c|c|c|c|c|}
\hline B & C & D & $\mathrm{E}$ & $F$ & G & H \\
\hline No & Time (sec) & Source Address (IPv6) & Destination Address (IPv6) & Protocol & $\begin{array}{l}\text { Length } \\
\text { (bytes) }\end{array}$ & Info \\
\hline 7948 & 1383.446 & fe80::212:7402:2:202 & fe80::212:7401:1:101 & ICMPv6 & 102 & RPL Control (DODAG Information Object) \\
\hline 7949 & 1383.446 & fe80::212:7402:2:202 & fe80::212:7401:1:101 & ICMPv6 & 102 & RPL Control (DODAG Information Object) \\
\hline 7950 & 1383.446 & fe80::212:7402:2:202 & fe80::212:7401:1:101 & ICMPv6 & 102 & RPL Control (DODAG Information Object) \\
\hline 7951 & 1383.446 & fe80::212:7402:2:202 & fe80::212:7401:1:101 & ICMPv6 & 102 & RPL Control (DODAG Information Object) \\
\hline 7952 & 1383.446 & fe80::212:7402:2:202 & fe80::212:7401:1:101 & ICMPv6 & 102 & RPL Control (DODAG Information Object) \\
\hline 7953 & 1383.446 & fe80::212:7402:2:202 & fe80::212:7401:1:101 & ICMPv6 & 102 & RPL Control (DODAG Information Object) \\
\hline 7954 & 1383.446 & fe80::212:7402:2:202 & fe80::212:7401:1:101 & ICMPv6 & 102 & RPL Control (DODAG Information Object) \\
\hline 7955 & 1383.446 & fe80::212:7402:2:202 & fe80::212:7401:1:101 & ICMPv6 & 102 & RPL Control (DODAG Information Object) \\
\hline 7956 & 1383.446 & fe80::212:7402:2:202 & fe80::212:7401:1:101 & ICMPv6 & 102 & RPL Control (DODAG Information Object) \\
\hline 7957 & 1383.446 & fe80::212:7402:2:202 & fe80::212:7401:1:101 & ICMPv6 & 102 & RPL Control (DODAG Information Object) \\
\hline 7958 & 1383.446 & fe80::212:7402:2:202 & fe80::212:7401:1:101 & ICMPv6 & 102 & RPL Control (DODAG Information Object) \\
\hline 7959 & 1383.446 & fe80::212:7402:2:202 & fe80::212:7401:1:101 & ICMPv6 & 102 & RPL Control (DODAG Information Object) \\
\hline 7960 & 1384.025 & fe80::212:7402:2:202 & fe80::212:7401:1:101 & ICMPv6 & 102 & RPL Control (DODAG Information Object) \\
\hline 7961 & 1384.025 & fe80::212:7402:2:202 & fe80::212:7401:1:101 & ICMPv6 & 102 & RPL Control (DODAG Information Object) \\
\hline 7962 & 1388.914 & fe $80:: 212: 7403: 3: 303$ & fe80::212:7401:1:101 & ICMPv6 & 102 & RPL Control (DODAG Information Object) \\
\hline 7963 & 1388.914 & fe80::212:7403:3:303 & fe80::212:7401:1:101 & ICMPv6 & 102 & RPL Control (DODAG Information Object) \\
\hline 7964 & 1388.914 & fe80::212:7403:3:303 & fe80::212:7401:1:101 & ICMPv6 & 102 & RPL Control (DODAG Information Object) \\
\hline 7965 & 1388.914 & fe80::212:7403:3:303 & fe80::212:7401:1:101 & ICMPv6 & 102 & RPL Control (DODAG Information Object) \\
\hline 7966 & 1389.531 & fe80::212:7403:3:303 & fe80::212:7401:1:101 & ICMPV6 & 102 & RPL Control (DODAG Information Object) \\
\hline 7967 & 1389.531 & fe80::212:7403:3:303 & fe80::212:7401:1:101 & ICMPv6 & 102 & RPL Control (DODAG Information Object) \\
\hline 7968 & 1389.531 & fe80::212:7403:3:303 & fe80::212:7401:1:101 & ICMPv6 & 102 & RPL Control (DODAG Information Object) \\
\hline 7969 & 1389.531 & fe $80:: 212: 7403: 3: 303$ & fe80::212:7401:1:101 & ICMPv6 & 102 & RPL Control (DODAG Information Object) \\
\hline 7970 & 1389.531 & fe $80:: 212: 7403: 3: 303$ & fe80::212:7401:1:101 & ICMPv6 & 102 & RPL Control (DODAG Information Object) \\
\hline 7971 & 1389.531 & fe80::212:7403:3:303 & fe80::212:7401:1:101 & ICMPv6 & 102 & RPL Control (DODAG Information Object) \\
\hline 7972 & 1389.531 & fe $80:: 212: 7403: 3: 303$ & fe80::212:7401:1:101 & ICMPv6 & 102 & RPL Control (DODAG Information Object) \\
\hline 7973 & 1389.532 & fe80::212:7403:3:303 & fe80::212:7401:1:101 & ICMPv6 & 102 & RPL Control (DODAG Information Object) \\
\hline 7974 & 1389.532 & fe80::212:7403:3:303 & fe80::212:7401:1:101 & ICMPv6 & 102 & RPL Control (DODAG Information Object) \\
\hline 7975 & 1389.532 & fe80::212:7403:3:303 & fe80::212:7401:1:101 & ICMPv6 & 102 & RPL Control (DODAG Information Object) \\
\hline
\end{tabular}

Figure 22. Benign "radiologICMPv6.csv"-7948 to 7975 records. 
"radiologUDP.csv": The generated benign "radiologUDP.csv" file consists of 104,048 records and its last 37 records (i.e., 104,012-104,048) are depicted below in Figure 23.

\begin{tabular}{|c|c|c|c|c|c|c|}
\hline B & c & D & E & $\mathrm{F}$ & G & H \\
\hline No & Time (sec) & Source Address (IPv6) & Destination Address (IPv6) & Protocol & Length (bytes) & Info \\
\hline 104012 & 5160.069 & $2002: d b 8:: 212: 7401: 1: 101$ & $2002: d b 8:: 212: 7404: 4: 404$ & UDP & 61 & Source port: rrac Destination port: ultraseek-http \\
\hline 104013 & 5228.195 & $2002: \mathrm{db} 8:: 212: 7401: 1: 101$ & $2002: d b 8:: 212: 7404: 4: 404$ & UDP & 61 & Source port: rrac Destination port: ultraseek-http \\
\hline 104014 & 5288.296 & $2002: d b 8:: 212: 7401: 1: 101$ & 2002:db8::212:7404:4:404 & UDP & 61 & Source port: rrac Destination port: ultraseek-http \\
\hline 104015 & 5338.452 & $2002: \mathrm{db} 8:: 212: 7401: 1: 101$ & $2002: d b 8:: 212: 7404: 4: 404$ & UDP & 61 & Source port: rrac Destination port: ultraseek-http \\
\hline 104016 & 5384.086 & $2002: \mathrm{db} 8:: 212: 7405: 5: 505$ & 2002:db8:ff:fe00:1 & UDP & 53 & Source port: ultraseek-http Destination port: rrac \\
\hline 104017 & 5404.824 & 2002:db8::212:7405:5:505 & 2002:db8:ff:fe00:1 & UDP & 53 & Source port: ultraseek-http Destination port: rrac \\
\hline 104018 & 5472.868 & $2002: \mathrm{db} 8:: 212: 7405: 5: 505$ & 2002:db8:ff:fe00:1 & UDP & 53 & Source port: ultraseek-http Destination port: rrac \\
\hline 104019 & 5499.575 & 2002:db8::212:7405:5:505 & 2002:db8:ff:fe00:1 & UDP & 53 & Source port: ultraseek-http Destination port: rrac \\
\hline 104020 & 5537 & $2002: d b 8:: 212: 7405: 5: 505$ & 2002:db8:ff:fe00:1 & UDP & 53 & Source port: ultraseek-http Destination port: rrac \\
\hline 104021 & 5577.016 & 2002:db8::212:7405:5:505 & 2002:db8:ff:fe00:1 & UDP & 53 & Source port: ultraseek-http Destination port: rrac \\
\hline 104022 & 5604.155 & 2002:db8::212:7405:5:505 & 2002:db8:ff:fe00:1 & UDP & 53 & Source port: ultraseek-http Destination port: rrac \\
\hline 104023 & 5641.794 & 2002:db8::212:7405:5:505 & 2002:db8:ff:fe00:1 & UDP & 53 & Source port: ultraseek-http Destination port: rrac \\
\hline 104024 & 5673.504 & 2002:db8::212:7405:5:505 & 2002:db8:ff:fe00:1 & UDP & 53 & Source port: ultraseek-http Destination port: rrac \\
\hline 104025 & 5705.082 & $2002: d b 8:: 212: 7405: 5: 505$ & 2002:db8::ff:fe00:1 & UDP & 53 & Source port: ultraseek-http Destination port: rrac \\
\hline 104026 & 5735.509 & $2002: \mathrm{db} 8:: 212: 7405: 5: 505$ & 2002:db8:ff:fe00:1 & UDP & 53 & Source port: ultraseek-http Destination port: rrac \\
\hline 104027 & 5771.839 & $2002: d b 8:: 212: 7405: 5: 505$ & 2002:db8:ff:fe00:1 & UDP & 53 & Source port: ultraseek-http Destination port: rrac \\
\hline 104028 & 5850.894 & $2002: \mathrm{db} 8:: 212: 7405: 5: 505$ & 2002:db8:ff:fe00:1 & UDP & 53 & Source port: ultraseek-http Destination port: rrac \\
\hline 104029 & 5877.398 & 2002:db8::212:7405:5:505 & 2002:db8:ff:fe00:1 & UDP & 53 & Source port: ultraseek-http Destination port: rrac \\
\hline 104030 & 5909.601 & $2002: \mathrm{db} 8:: 212: 7405: 5: 505$ & 2002:db8:ff:fe00:1 & UDP & 53 & Source port: ultraseek-http Destination port: rrac \\
\hline 104031 & 5936.792 & 2002:db8::212:7405:5:505 & 2002:db8:ff:fe00:1 & UDP & 53 & Source port: ultraseek-http Destination port: rrac \\
\hline 104032 & 5967.579 & $2002: d b 8:: 212: 7405: 5: 505$ & 2002:db8:ff:fe00:1 & UDP & 53 & Source port: ultraseek-http Destination port: rrac \\
\hline 104033 & 5994.686 & 2002:db8::212:7405:5:505 & 2002:db8:ff:fe00:1 & UDP & 53 & Source port: ultraseek-http Destination port: rrac \\
\hline 104034 & 6027.008 & 2002:db8::212:7405:5:505 & 2002:db8:ff:fe00:1 & UDP & 53 & Source port: ultraseek-http Destination port: rrac \\
\hline 104035 & 6059.489 & 2002:db8::212:7405:5:505 & 2002:db8:ff:fe00:1 & UDP & 53 & Source port: ultraseek-http Destination port: rrac \\
\hline 104036 & 6094.091 & 2002:db8::212:7405:5:505 & 2002:db8:ff:fec0:1 & UDP & 53 & Source port: ultraseek-http Destination port: rrac \\
\hline 104037 & 6149.474 & 2002:db8::212:7405:5:505 & 2002:db8::ff:fe00:1 & UDP & 53 & Source port: ultraseek-http Destination port: rrac \\
\hline 104038 & 6185.05 & $2002: d b 8:: 212: 7405: 5: 505$ & 2002:db8::ff:fe00:1 & UDP & 53 & Source port: ultraseek-http Destination port: rrac \\
\hline 104039 & 6245.208 & $2002: d b 8:: 212: 7405: 5: 505$ & 2002:db8::ff:fe00:1 & UDP & 53 & Source port: ultraseek-http Destination port: rrac \\
\hline 104040 & 6279.464 & 2002:db8::212:7405:5:505 & 2002:db8::ff:fe00:1 & UDP & 53 & Source port: ultraseek-http Destination port: rrac \\
\hline 104041 & 6316.108 & $2002: \mathrm{db} 8:: 212: 7405: 5: 505$ & 2002:db8::ff:fe00:1 & UDP & 53 & Source port: ultraseek-http Destination port: rrac \\
\hline 104042 & 6362.969 & $2002: \mathrm{db} 8:: 212: 7405: 5: 505$ & 2002:db8:ff:fe00:1 & UDP & 53 & Source port: ultraseek-http Destination port: rrac \\
\hline 104043 & 6393.244 & 2002:db8::212:7405:5:505 & 2002:db8:ff:fe00:1 & UDP & 53 & Source port: ultraseek-http Destination port: rrac \\
\hline 104044 & 6427.186 & $2002: \mathrm{db} 8:: 212: 7405: 5: 505$ & 2002:db8:ff:fe00:1 & UDP & 53 & Source port: ultraseek-http Destination port: rrac \\
\hline 104045 & 6457.901 & $2002: \mathrm{db} 8:: 212: 7405: 5: 505$ & 2002:db8:ff:fe00:1 & UDP & 53 & Source port: ultraseek-http Destination port: rrac \\
\hline 104046 & 6522.564 & $2002: \mathrm{db} 8:: 212: 7405: 5: 505$ & 2002:db8:ff.fe00:1 & UDP & 53 & Source port: ultraseek-http Destination port: rrac \\
\hline 104047 & 6591.672 & $2002: \mathrm{db} 8:: 212: 7405: 5: 505$ & 2002:db8:ff:fe00:1 & UDP & 53 & Source port: ultraseek-http Destination port: rrac \\
\hline 104048 & 6647.425 & $2002: \mathrm{db} 8:: 212: 7405: 5: 505$ & 2002:db8:ff:fe00:1 & UDP & 53 & Source port: ultraseek-http Destination port: rrac \\
\hline
\end{tabular}

Figure 23. Benign "radiologUDP.csv"-104,012 to 104,048 records.

\section{Generation of Malicious IoT/IIoT Datasets}

In this Section, we provide a detailed description of the approach followed to generate a set of malicious datasets by implementing a UDP flooding attack scenario in the Cooja simulator, as shown in Figure 24. Similar to the approach followed for the generation of the benign datasets in Section 4, the generated IoT/IIoT-specific information from the simulated attack scenario was captured from the Contiki plugin "powertrace" (i.e., features such as CPU consumption) and the Cooja tool "Radio messages" (i.e., network traffic features) in order to generate the "powertrace" dataset and the network traffic dataset for the simulated UDP flooding attack scenario.

The network topology of the simulated UDP flooding attack scenario in the Cooja simulator environment consists of 4 yellow (benign) UDP-client motes (i.e., motes 2, 3, 4 and 5), the violet (malicious) UDP-client mote (i.e., mote 6) and the green (benign) UDPsever mote (i.e., mote 1), as depicted in Figure 24. The simulation duration was set to $60 \mathrm{~min}$ and the motes' outputs were printed out in the respective window (e.g., Mote output) while simulations run, as shown in Figure 25. Moreover, the 4 yellow (benign) UDP-client motes were configured to send text messages every $10 \mathrm{~s}$, approximately, to the UDP-sever mote that was configured to provide a corresponding response. On the other hand, the violet (malicious) UDP-client mote (i.e., mote 6) was compromised with malicious code in order to send UDP packets within a very short period of time (i.e., every $200 \mathrm{~ms}$ ). Finally, it is noteworthy to say that similar to the benign network scenario, the UDP protocol was used at the Transport Layer, the IPv6 at the network layer, and the type of motes was the Tmote Sky in the UDP flooding attack scenario. 


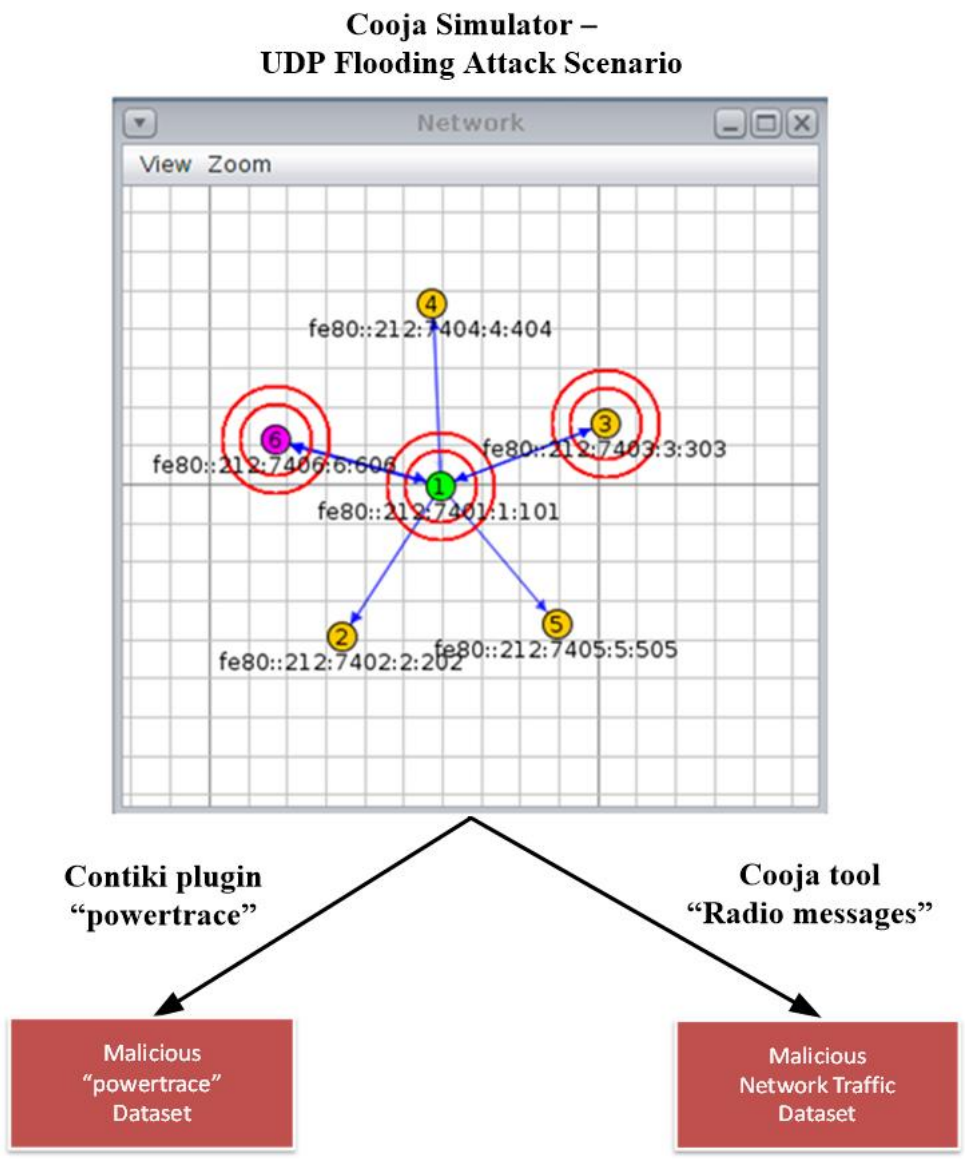

Figure 24. Malicious datasets generation by utilizing the Cooja simulator.

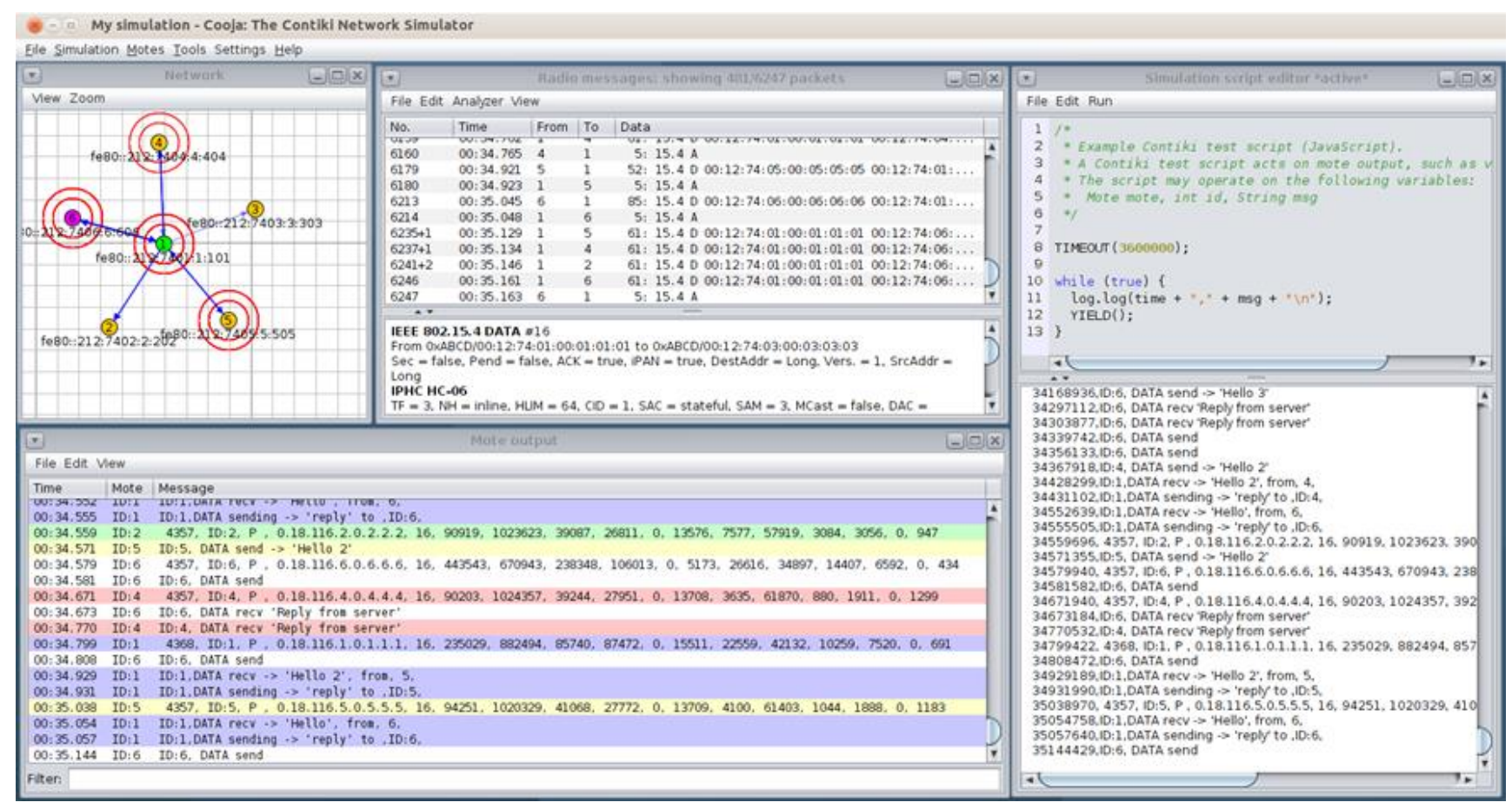

Figure 25. Cooja Simulator-motes' outputs. 


\subsection{Malicious "Powertrace" Dataset Generation \\ 5.1.1. Malicious "Powertrace" Dataset Generation}

The approach followed for the "powertrace" dataset generation from the UDP flooding attack scenario was similar to the approach followed for the "powertrace" dataset generation from the benign IIoT network scenario in Section 4.1.1. In addition, the "powertrace" plugin was similarly enabled for collecting "powertrace" related features, summarised in Table 1, from the motes of the attack scenario every two seconds. In Figure 26, the depicted mote output window displays the captured "powertrace" information every two seconds and also the messages sent and received by each mote during the simulation time (60 $\mathrm{min})$.

\begin{tabular}{|c|c|c|c|}
\hline$\nabla$ & & Mote output & - \\
\hline \multicolumn{4}{|c|}{ File Edit View } \\
\hline Time & Mote & Message & \\
\hline $00: 28.442$ & ID: 6 & ID: 6 , DATA send & $\Delta$ \\
\hline $00: 28.559$ & ID: 2 & 3589, ID:2, P, $0.18 .116 .2 .0 .2 .2 .2,13,66166,851855,26911,19241,0,10714,15532,49976,9118,4676,0,893$ & T \\
\hline $00: 28.575$ & ID: 6 & 3589, ID:6, P, 0.18.116.6.0.6.6.6, 13, 369509, 548346, 200169, 87513,0, 4226, 26088, 38972, 14483, 6438, 0,216 & \\
\hline $00: 28.577$ & ID: 6 & ID:6, DATA send & \\
\hline $00: 28.671$ & ID $: 4$ & 3589, ID:4, P, 0.18.116.4.0.4.4.4, 13, 77453,840576,34802, 23753,0,10529,6336, 59173, 3040, 2197, 0,947 & \\
\hline $00: 28.706$ & ID:1 & 3589, ID:1, P, 0.18.116.1.0.1.1.1, 13, 175491, 742504, 59560, 66656, 0, 12931, 14597, 50506, 6062, 5242, 0, 1492 & \\
\hline $00: 28.777$ & ID: 6 & ID: 6 , DATA send & \\
\hline $00: 28.996$ & ID: 6 & ID:6, DATA send & \\
\hline $00: 29.038$ & ID:5 & 3589 , ID:5, P, $0.18 .116 .5 .0 .5 .5 .5,13,82239,835811,37031,23511,0,10233,5129,60378,2163,1521,0,581$ & U \\
\hline $00: 29.105$ & ID: 6 & ID: 6 , DATA send & - \\
\hline
\end{tabular}

Figure 26. Cooja Simulator-Mote output window.

When the timeout occurred, the simulation stopped, and all the captured information and prints were stored in the "COOJA.testlog" file. Afterwards, the "IoT_Simul.sh" file, described in Section 4.1.1, created (a) a new root folder named as "2020-12-09-14-59-59", and (b) the "log" folder, inside the "2020-12-09-14-59-59" folder, where the "COOJA.testlog" file was copied from the "... / cooja/build" folder located in the Cooja Simulator. Then, the "IoT_Simul.sh" file following the same process, as described in Section 4.1.1, extracted the required "powertrace" information from the "COOJA.testlog" file and saved it in the "pwrtrace.csv" file in the "dataset" folder that was created by the batch file inside the "2020-12-09-14-59-59" folder, as shown below in the left part of Figure 27. In the "dataset" folder, apart from the "pwrtrace.csv" file, the "IoT_Simul.sh" file generated two more files (i.e., the "recv.csv" file and the "send.csv"), following the same process as in Section 4.1.1. The "recv.csv" file and the "send.csv" file include the "received" and "sent" messages printed by the motes, respectively.

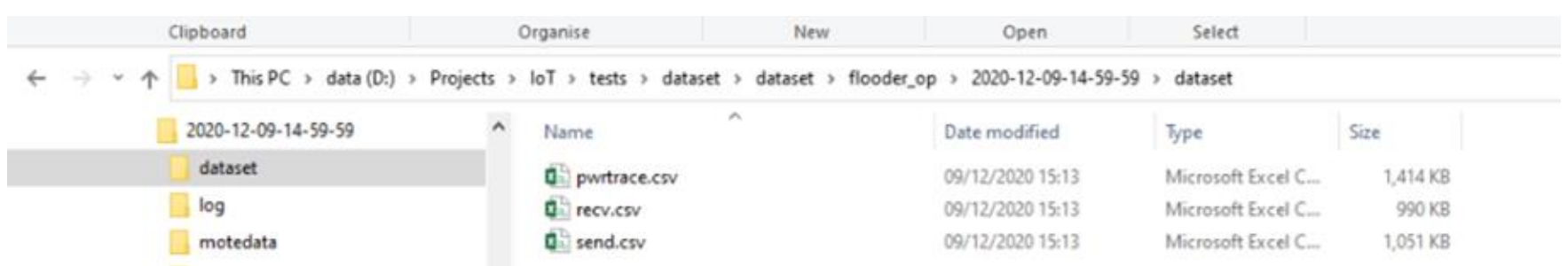

Figure 27. Location of the generated "pwrtrace.csv", "recv.csv", and "send.csv" files by the "IoT_Simul.sh" bash file.

Finally, similar to the benign "powertrace" dataset generation approach in Section 4.1.1, the "IoT_Simul.sh" file extracted the information related to each mote from the "pwrtrace.csv" file and generated one csv file for each mote with the corresponding information from the "pwrtrace.csv" file. The generated six csv files (i.e., mote1.csv, mote2.csv, mote3.csv, mote4.csv, mote5.csv, and mote6.csv) were stored in the "motedata" folder, created also by the "IoT_Simul.sh" file, as shown in the left part of Figure 27. 


\subsubsection{Malicious "powertrace" Datasets-Results}

Malicious "pwrtrace.csv": The generated malicious "pwrtrace.csv" file consists of $10,794$ records and its first 38 records (i.e., $1-38)$ and its last 38 records $(10,757-10,794)$ are depicted in Figures 28 and 29, respectively.

\begin{tabular}{|c|c|c|c|c|c|c|c|c|c|c|c|c|c|c|c|c|c|c|}
\hline \multirow[b]{3}{*}{ No } & D & E & $\mathrm{F}$ & G & H & I & $\mathrm{k}$ & L & M & $\mathrm{N}$ & 0 & p & R & s & T & u & $\mathrm{v}$ & w \\
\hline & & & & & & & \multicolumn{6}{|c|}{ Total measurements from the begining of the simulation } & \multicolumn{6}{|c|}{ Measurements for each of the 2-sec monitoring period } \\
\hline & $\begin{array}{c}\text { Real time } \\
\text { [us] }\end{array}$ & \begin{tabular}{|c|} 
clock time \\
(in ticks)
\end{tabular} & 10 & & Rime Address & sea no & $\begin{array}{l}\text { all_cpu } \\
\text { (in ticks) }\end{array}$ & $\begin{array}{l}\text { all_lpm } \\
\text { (in ticks) }\end{array}$ & \begin{tabular}{|l|} 
all_transmit \\
(in ticks)
\end{tabular} & $\begin{array}{l}\text { allllisten } \\
\text { (in ticks) }\end{array}$ & $\begin{array}{c}\text { all_jidle_transmit } \\
\text { (in ticks) }\end{array}$ & \begin{tabular}{|l} 
all jidle_listen \\
(in ticks)
\end{tabular} & \begin{tabular}{|c|} 
cpu \\
(in ticks)
\end{tabular} & \begin{tabular}{|c|} 
Ipm \\
(in ticks)
\end{tabular} & \begin{tabular}{|l} 
transmit \\
(in ticks)
\end{tabular} & \begin{tabular}{|c|} 
listen \\
(in ticks) \\
\end{tabular} & \begin{tabular}{|c} 
idle_transmit \\
(in ticks)
\end{tabular} & \begin{tabular}{|l} 
idle_listen \\
(in ticks)
\end{tabular} \\
\hline 1 & 2555692 & 261 & 10.2 & $P$ & 0.18 .116 .20 .2 .22 & 0 & 6742 & 59714 & 2589 & 442 & 0 & 364 & 6742 & 59714 & 2589 & 442 & 0 & 364 \\
\hline 2 & 2570487 & 261 & 10.6 & $P$ & 0.18 .116 .60 .6 .6 .6 & 0 & 7709 & 58725 & 2590 & 442 & 0 & 364 & 7709 & 58725 & 2590 & 442 & 0 & 364 \\
\hline 3 & 2665753 & 261 & 10.4 & $p$ & 0.18 .116 .40 .4 .4 .4 & 0 & 2189 & 64265 & 0 & 390 & 0 & 390 & 2189 & 64265 & 0 & 390 & 0 & 390 \\
\hline 4 & 2699493 & 261 & $10: 1$ & $p$ & 0.18 .116 .10 .1 .11 & 0 & 2817 & 63639 & 0 & 999 & 0 & 744 & 2817 & 63639 & 0 & 999 & 0 & 744 \\
\hline 5 & 3034683 & 261 & 105 & $P$ & 0.18 .116 .50 .5 .55 & 0 & 6742 & 59714 & 2589 & 442 & 0 & 364 & 6742 & 59714 & 2589 & 442 & 0 & 364 \\
\hline 6 & 3216735 & 261 & 10.3 & $p$ & 0.18 .116 .30 .3 .3 .3 & 0 & 2189 & 64265 & 0 & 390 & 0 & 390 & 2189 & 64265 & 0 & 390 & 0 & 390 \\
\hline 7 & 4554978 & 517 & 10.2 & $P$ & 0.18 .116 .20 .2 .2 .2 & 1 & 7904 & 124063 & 2589 & 858 & 0 & 780 & 1159 & 64349 & 0 & 416 & 0 & 416 \\
\hline 8 & 4575548 & 517 & 10.6 & $P$ & 0.18 .116 .60 .6 .6 .6 & 1 & 10228 & 121854 & 2590 & 1159 & 0 & 767 & 2516 & 63129 & 0 & 717 & 0 & 403 \\
\hline 9 & 4671767 & 517 & 10.4 & $P$ & 0.18 .116 .40 .4 .4 .4 & $\frac{4}{1}$ & 3574 & 128552 & 0 & 1104 & 0 & 1056 & 1382 & 64287 & 0 & 714 & 0 & 666 \\
\hline 10 & 4702609 & 517 & $10: 1$ & $D$ & 0.18 .116 .10 .1 .1 .1 & 1 & 8551 & 123417 & 2980 & 1467 & 0 & 1134 & 5731 & 59778 & 2980 & 468 & 0 & 390 \\
\hline 11 & 5034813 & 517 & 10.5 & $D$ & 0.18 .116 .50 .5 .55 & 1 & 8255 & 123715 & 2589 & 1136 & 0 & 1010 & 1510 & 64001 & 0 & 694 & 0 & 645 \\
\hline 12 & 5217991 & 517 & 10.3 & $p$ & 0.18 .116 .30 .3 .33 & 1 & 3658 & 128314 & 0 & 1090 & 0 & 1043 & 1466 & 64049 & 0 & 700 & 0 & 653 \\
\hline 13 & 6555863 & 773 & 10.2 & $p$ & 0.18 .116 .20 .2 .22 & 2 & 9577 & 187908 & 2589 & 1471 & 0 & 1170 & 1670 & 63845 & 0 & 613 & 0 & 390 \\
\hline 14 & 6573145 & 773 & 10.6 & $p$ & 0.18 .116 .60 .6 .6 .6 & 2 & 40545 & 156877 & 20450 & 8529 & 0 & 988 & 30315 & 35023 & 17860 & 7370 & 0 & 221 \\
\hline 15 & 6666980 & 773 & 10.4 & $p$ & 0.18 .116 .40 .4 .4 .4 & 2 & 4960 & 192521 & 0 & 1520 & 0 & 1472 & 1383 & 63969 & 0 & 416 & 0 & 416 \\
\hline 16 & 6704432 & 773 & $10: 1$ & $P$ & 0.18 .116 .10 .1 .11 & 2 & 12693 & 184842 & 2980 & 3685 & 0 & 2194 & 4140 & 61425 & 0 & 2218 & 0 & 1060 \\
\hline 17 & 7036198 & 773 & 105 & $P$ & 0.18 .116 .50 .5 .55 & 2 & 14278 & 183202 & 5575 & 1605 & 0 & 1400 & 6020 & 59487 & 2986 & 469 & 0 & 390 \\
\hline 18 & 7217945 & 773 & 10.3 & $p$ & 0.18 .116 .30 .3 .3 .3 & 2 & 5047 & 192434 & 0 & 1506 & 0 & 1459 & 1386 & 64120 & 0 & 416 & 0 & 416 \\
\hline 19 & 8557499 & 1029 & 10.2 & $P$ & 0.18 .116 .20 .2 .2 .2 & 3 & 15580 & 247416 & 5574 & 1940 & 0 & 1560 & 6000 & 59508 & 2985 & 469 & 0 & 390 \\
\hline 20 & 8574202 & 1029 & 10.6 & $P$ & 0.18 .116 .60 .6 .6 .6 & 3 & 72195 & 190733 & 39240 & 14939 & 0 & 1222 & 31648 & 33856 & 18790 & 6410 & 0 & 234 \\
\hline 21 & 8670462 & 1029 & $10: 4$ & $P$ & 0.18 .116 .40 .4 .4 .4 & 3 & 21137 & 241852 & 9460 & 4759 & 0 & 1810 & 16174 & 49331 & 9460 & 3239 & 0 & 338 \\
\hline 22 & 8702861 & 1029 & $10: 1$ & $P$ & 0.18 .116 .10 .1 .11 & 3 & 15882 & 247108 & 2980 & 6503 & 0 & 3838 & 3186 & 62266 & 0 & 2818 & 0 & 1644 \\
\hline 23 & 9037531 & 1029 & 10.5 & $P$ & 0.18 .116 .50 .5 .55 & 3 & 25136 & 237851 & 11495 & 4573 & 0 & 1738 & 10855 & 54649 & 5920 & 2968 & 0 & 338 \\
\hline 24 & \begin{tabular}{|l}
9221415 \\
\end{tabular} & 1029 & 10.3 & $p$ & 0.18 .116 .30 .3 .3 .3 & 3 & 19298 & 243688 & 8345 & 4245 & 0 & 1823 & 14248 & 51254 & 8345 & 2739 & 0 & 364 \\
\hline 25 & 10558220 & 1285 & 10.2 & $P$ & 0.18 .116 .20 .2 .22 & 4 & 25340 & 303155 & 10934 & 4604 & 0 & 1924 & 9757 & 55739 & 5360 & 2664 & 0 & 364 \\
\hline 26 & 10574593 & 1285 & 10.6 & $p$ & 0.18 .116 .60 .6 .6 .6 & 4 & 102520 & 225896 & 56293 & 22039 & 0 & 1456 & 30322 & 35163 & 17053 & 7100 & 0 & 234 \\
\hline 27 & \begin{tabular}{|l|l|}
10668636 \\
\end{tabular} & 1285 & 10.4 & $p$ & 0.18 .116 .40 .4 .44 & 4 & 22607 & 305875 & 9460 & 5175 & 0 & 2226 & 1468 & 64023 & 0 & 416 & 0 & 416 \\
\hline 28 & 10707377 & 1285 & $10: 1$ & $P$ & 0.18 .116 .10 .1 .1 .1 & 4 & 21475 & 307168 & 2980 & 10148 & 0 & 4695 & 5590 & 60060 & 0 & 3645 & 0 & 857 \\
\hline 29 & 11035707 & 1285 & 10.5 & $P$ & 0.18 .116 .50 .5 .55 & 4 & 26575 & 301905 & 11495 & 4989 & 0 & 2154 & 1437 & 64054 & 0 & 416 & 0 & 416 \\
\hline 30 & 11219597 & 1285 & 10.3 & $P$ & 0.18 .116 .30 .3 .3 .3 & 4 & 20726 & 307753 & 8345 & 4661 & 0 & 2239 & 1426 & 64065 & 0 & 416 & 0 & 416 \\
\hline 31 & 12557488 & 1541 & 102 & $P$ & 0.18 .116 .20 .2 .22 & 5 & 27170 & 366840 & 10934 & 5773 & 0 & 3048 & 1828 & 63685 & 0 & 1169 & 0 & 1124 \\
\hline 32 & 12669462 & 1541 & 10.4 & $P$ & 0.18 .116 .4 .0 .4 .4 .4 & 5 & 24354 & 369643 & 9460 & 6363 & 0 & 3383 & 1745 & 63768 & 0 & 1188 & 0 & 1157 \\
\hline 33 & 12700632 & 1557 & 10.6 & $p$ & 0.18 .116 .60 .6 .66 & 5 & 134474 & 263557 & 73964 & 30327 & 0 & 1940 & 31951 & 37661 & 17671 & 8288 & 0 & 484 \\
\hline 34 & $\begin{array}{l}12821697 \\
\end{array}$ & 1556 & $10: 1$ & $p$ & 0.18 .116 .10 .1 .1 .1 & 5 & 45913 & 351915 & 15135 & 17366 & 0 & 5621 & 24436 & 44747 & 12155 & 7218 & 0 & 926 \\
\hline 35 & 13036484 & 1541 & 105 & $P$ & 0.18 .116 .50 .5 .55 & 5 & 28370 & 365626 & 11495 & 6481 & 0 & 3331 & 1792 & 63721 & 0 & 1492 & 0 & 1177 \\
\hline 36 & 13219734 & 1541 & 10.3 & $p$ & 0.18 .116 .30 .3 .3 .3 & 5 & 22495 & 371498 & 8345 & 5528 & 0 & 2806 & 1766 & 63745 & 0 & 867 & 0 & 567 \\
\hline 37 & 14557450 & 1797 & 10.2 & $p$ & 0.18 .116 .20 .2 .22 & 6 & 28686 & 430834 & 10934 & 6773 & 0 & 4048 & 1513 & 63994 & 0 & 1000 & 0 & 1000 \\
\hline 38 & 14590601 & 1799 & 106 & $p$ & 01811660666 & 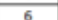 & 167799 & 292124 & 92841 & 37747 & 0 & 2083 & 33323 & 28567 & 18877 & 7420 & 0 & 143 \\
\hline
\end{tabular}

Figure 28. Malicious "pwrtrace.csv" -1 to 38 records.

\begin{tabular}{|c|c|c|c|c|c|c|c|c|c|c|c|c|c|c|c|c|c|c|}
\hline \multirow[b]{3}{*}{ No } & D & E & $\mathrm{F}$ & G & H & 1 & $\mathrm{k}$ & L & M & $\mathrm{N}$ & 0 & $\mathbf{p}$ & R & 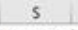 & T & u & $\mathrm{v}$ & w \\
\hline & & & & & & & \multicolumn{6}{|c|}{ Total measurements from the begining of the simulation } & \multicolumn{6}{|c|}{ Messurements for each of the 2 -sec monit toring period } \\
\hline & $\begin{array}{c}\text { Real time } \\
\text { [us] }\end{array}$ & $\begin{array}{l}\text { Clock time } \\
\text { (in ticks) }\end{array}$ & ID & & Rime Address & sea no & $\begin{array}{l}\text { all_cpu } \\
\text { (in ticks) }\end{array}$ & $\begin{array}{l}\text { All.Jpm } \\
\text { (in ticks) }\end{array}$ & \begin{tabular}{|c|} 
ollittransmit \\
(in ticks)
\end{tabular} & \begin{tabular}{|l|} 
all listen \\
(in ticks)
\end{tabular} & $\begin{array}{l}\text { oll_idile_transmit } \\
\text { (in ticks) }\end{array}$ & $\begin{array}{l}\text { all jiolle Jisten } \\
\text { (in ticks) }\end{array}$ & \begin{tabular}{|c|} 
cpu \\
(in ticks)
\end{tabular} & \begin{tabular}{|c|} 
Ipm \\
(in ticks)
\end{tabular} & \begin{tabular}{|l|} 
transmit \\
(in ticks)
\end{tabular} & \begin{tabular}{|c|} 
listen \\
(in ticks)
\end{tabular} & $\begin{array}{l}\text { idle,transmit } \\
\text { (in ticks) }\end{array}$ & $\begin{array}{l}\text { idle flisten } \\
\text { (in ticks) }\end{array}$ \\
\hline 10757 & $3587 \mathrm{E}+09$ & 459018 & 10.5 & P & 0.18 .1165 .0 .55 .5 & 1792 & 6484924 & 110972410 & 1976106 & 3249351 & 0 & 2067142 & 13864 & 52920 & 7065 & 5266 & 0 & 1092 \\
\hline 10758 & $3.587 E+09$ & 459013 & 10:3 & $P$ & 0.18 .116 .3 .0 .3 .3 .3 & 1792 & 6407343 & 111044806 & 1988080 & 2342008 & 0 & 1181632 & 1615 & 63875 & 0 & 426 & 0 & 416 \\
\hline 10759 & $3.589 E+09$ & 459269 & $10: 2$ & $P$ & 0.18 .116 .02 .2 .2 & 1793 & 6288419 & 111233629 & 1859570 & 3180790 & 0 & 2071651 & 10964 & 54533 & 5355 & 4048 & 0 & 908 \\
\hline 10760 & $3.5896+09$ & 459269 & $10: 6$ & $P$ & 0.18 .116 .0 .6 .6 .6 & 1793 & 49272148 & 68222749 & \begin{tabular}{|l|l|}
26428032 \\
\end{tabular} & 12698225 & 0 & 487982 & 21797 & 41017 & 11343 & 5525 & 0 & 234 \\
\hline 10761 & $3.589 \mathrm{E}+09$ & 459269 & $10: 4$ & $p$ & $0.18,116,4.04 .44$ & 1793 & 6077237 & 111445104 & 1735004 & 3122961 & 0 & 2078867 & 1654 & 63857 & 0 & 960 & 0 & 960 \\
\hline 10762 & $3.5895+09$ & 459269 & $10: 1$ & $p$ & 0.18 .116 .0 .1 .1 .1 & 1793 & 37354505 & 80163010 & \begin{tabular}{|l|l|}
16447901 \\
\end{tabular} & 12709259 & 0 & 1274462 & 15538 & 49969 & 6420 & 5486 & 0 & 976 \\
\hline 10763 & $3.589 t+09$ & 459269 & 10.5 & $p$ & 0.18 .1165 .05 .55 & 1793 & \begin{tabular}{|l|l|}
6486773 \\
\end{tabular} & 111034789 & 1976106 & 3250322 & 0 & 2067906 & 1846 & 62379 & 0 & 971 & 0 & 764 \\
\hline 10764 & $3.5895+09$ & 459269 & $10: 3$ & $p$ & 0.181163 .033 .3 & 1793 & 6408983 & 111108661 & 1988080 & 2342621 & 0 & 1182245 & 1637 & 63855 & 0 & 613 & 0 & 613 \\
\hline 10765 & $3.591 E+09$ & 459525 & 10.2 & $P$ & 0.18 .1162 .0 .2 .2 .2 & 1794 & 6293423 & 111294132 & \begin{tabular}{|l|}
1861337 \\
\end{tabular} & 3182661 & 0 & 2072205 & 5002 & 60503 & 1767 & 1871 & 0 & 554 \\
\hline 10766 & $3.591 E+09$ & 459528 & 10.6 & $p$ & 0.18 .116 .6 .0 .6 .6 .6 & 1794 & \begin{tabular}{|l|}
49303975 \\
\end{tabular} & 68257291 & 26445531 & 12706237 & 0 & 488374 & 31824 & 34542 & 17499 & 8012 & 0 & 392 \\
\hline 10767 & $3.591 E+09$ & 459525 & ID. 4 & $P$ & 0.18 .116 .40 .4 .44 & 1794 & \begin{tabular}{|l|}
6078847 \\
\end{tabular} & 111509005 & \begin{tabular}{|l|}
1735004 \\
\end{tabular} & 3123744 & 0 & 2079650 & 1607 & 63901 & 0 & 783 & 0 & 783 \\
\hline 10768 & $3.591 E+09$ & 459540 & ID:1 & $P$ & 0.18 .116 .0 .1 .1 .1 & 1794 & 37376330 & 80210698 & \begin{tabular}{|l|l|}
16456056 \\
\end{tabular} & 12717221 & 0 & 1274968 & 21822 & 47688 & 8155 & 7962 & 0 & 506 \\
\hline 10769 & $3.591 \mathrm{E}+09$ & 459525 & 10.5 & $p$ & 0.18 .1165 .0555 & 1794 & \begin{tabular}{|l|}
6488393 \\
\end{tabular} & 211098680 & \begin{tabular}{|l|}
1976106 \\
\end{tabular} & 3251466 & 0 & 2069050 & 1617 & 63891 & 0 & 1144 & 0 & 2144 \\
\hline 10770 & $3.591 E+09$ & 459525 & $10: 3$ & $P$ & 0.18 .1163 .0 .33 .3 & 1794 & 6413239 & 111169907 & \begin{tabular}{|l|}
1989162 \\
\end{tabular} & 2344529 & 0 & 2183238 & 4253 & 61246 & 1082 & 1908 & 0 & 993 \\
\hline 10771 & $3.593 \mathrm{E}+09$ & 459781 & $10: 2$ & $P$ & 0.18 .1162 .0 .2 .2 .2 & 1795 & 6295156 & 111357899 & 1851337 & 3183818 & 0 & 2073362 & 1730 & 63767 & 0 & 1157 & 0 & 1157 \\
\hline 10772 & $3593 \mathrm{E}+09$ & 459782 & 10.6 & $p$ & 0.18 .116 .6 .0 .6 .6 .6 & 1795 & 49329509 & 68296718 & 26458484 & 12713264 & 0 & 488746 & 25532 & 39427 & 12953 & 7027 & 0 & 372 \\
\hline 10773 & $3.593 E+09$ & 459781 & $10: 4$ & $p$ & 0.18 .116 .40 .4 .4 .4 & 1795 & 6080517 & 111572831 & 1735004 & 3125078 & 0 & 2080984 & 1667 & 63826 & 0 & 1334 & 0 & 1334 \\
\hline 10774 & $35936+09$ & 459781 & $\mathrm{DD}: 1$ & $p$ & $0.18 .116 \cdot 10.1 .1 .1$ & 1795 & 37397949 & 80250568 & \begin{tabular}{|l|l|}
16465241 \\
\end{tabular} & 12724409 & 0 & 1275229 & 21616 & 39870 & 9185 & 7188 & 0 & 261 \\
\hline 10775 & $3593 E+09$ & 459781 & 10.5 & $p$ & 0.18 .1165055 .5 & 1795 & 6490075 & 111162496 & 1976106 & 3252623 & 0 & 2070207 & 1679 & 63816 & 0 & 1157 & 0 & 1157 \\
\hline 10776 & $3593 E+09$ & 459781 & $10: 3$ & $P$ & 0.18 .116 .3 .0 .3 .3 .3 & 1795 & \begin{tabular}{|l|l|}
6416946 \\
\end{tabular} & 111233697 & \begin{tabular}{|l|}
1989162 \\
\end{tabular} & 2345345 & 0 & 1184054 & 1704 & 63790 & 0 & 816 & 0 & 816 \\
\hline 10777 & $3.595 E+09$ & 460037 & 10.2 & $P$ & 0.18 .1162 .02 .22 & 1796 & \begin{tabular}{|l|}
6296887 \\
\end{tabular} & 111421667 & 1861337 & 3185493 & 0 & 2075037 & 1728 & 63768 & 0 & 1675 & 0 & 1675 \\
\hline 10778 & $3.595 \mathrm{E}+09$ & 460037 & 10.6 & $p$ & 0.18 .116 .60 .6 .6 .6 & 1796 & 49355789 & 683335752 & 26472262 & 12720153 & 0 & 488850 & 26277 & 39034 & 13778 & 6889 & 0 & 104 \\
\hline 10779 & $3.595 E+09$ & 460037 & 10.4 & $P$ & 0.18 .116 .40 .4 .44 & 1796 & 6082233 & 111636611 & \begin{tabular}{|l|}
1735004 \\
\end{tabular} & 3126792 & 0 & 2082698 & 1713 & 63780 & 0 & 1714 & 0 & 1714 \\
\hline 10780 & $3.595 \mathrm{E}+09$ & 460037 & $10: 2$ & $P$ & 0.18 .1261 .0 .1 .1 .1 & 1796 & 37428570 & 80285455 & \begin{tabular}{|l|l|}
16481427 \\
\end{tabular} & 12733982 & 0 & 1275681 & 30619 & 34887 & 16186 & 9573 & 0 & 452 \\
\hline 10781 & $3.595 \mathrm{E}+09$ & 460037 & 10.5 & $P$ & 0.18 .1165 .0555 & 1796 & 6491782 & 111226285 & 1976106 & 3254337 & 0 & 2071921 & 1704 & 63789 & 0 & 1714 & 0 & 1714 \\
\hline 10782 & $3.595 E+09$ & 460037 & 1D:3 & $p$ & 0.18 .1163 .03 .3 .3 & 1796 & 6416585 & 111297551 & 1989162 & 2346148 & 0 & 1184857 & 1636 & 63854 & 0 & 803 & 0 & 803 \\
\hline 10783 & $3.597 E+09$ & 460293 & $10: 2$ & $p$ & 0.18 .1162 .02 .2 .2 & 1797 & \begin{tabular}{|l|}
6303068 \\
\end{tabular} & 111480993 & 1863731 & 3188667 & 0 & 2076549 & 6178 & 59326 & 2394 & 3174 & 0 & 1512 \\
\hline 10784 & $3597 \mathrm{E}+09$ & 460296 & 10.6 & $p$ & 0.18 .116 .6 .0 .6 .6 .6 & 1797 & 49386703 & 68371029 & 26489236 & 12727987 & 0 & 489439 & 30911 & 35277 & 16974 & 7834 & 0 & 589 \\
\hline 10785 & $3.597 E+09$ & 460293 & $10: 4$ & $P$ & 0.18 .116 .4 .0 .4 .44 & 1797 & 6088623 & 111695721 & \begin{tabular}{|l|}
1737682 \\
\end{tabular} & 3129879 & 0 & 2034216 & 6387 & 59110 & 2678 & 3087 & 0 & 1518 \\
\hline 10786 & $3.597 E+09$ & 460293 & $10: 1$ & $p$ & $0.18 \cdot 116 \cdot 1.01 .1 .1$ & 1797 & 37456122 & 80323411 & \begin{tabular}{|l|l|}
16495172 \\
\end{tabular} & 12743176 & 0 & 1276539 & 27549 & 37956 & 13745 & 9194 & 0 & 858 \\
\hline 10787 & $3.597 \mathrm{E}+09$ & 460293 & 10.5 & $p$ & 0.18 .1165 .0555 & 1797 & \begin{tabular}{|l|}
6493481 \\
\end{tabular} & 111290084 & \begin{tabular}{|l|}
1976106 \\
\end{tabular} & 3255887 & 0 & 2073471 & 1696 & 63799 & 0 & 1550 & 0 & 1550 \\
\hline 10788 & \begin{tabular}{|l|l}
$3.597 \mathrm{E}+09$ \\
\end{tabular} & 460293 & $10: 3$ & $P$ & 0.18 .116 .3 .0 .3 .3 .3 & 1797 & 6427436 & 111352202 & 1994524 & 2350173 & 0 & 1185746 & 10848 & 54651 & 5362 & 4025 & 0 & 889 \\
\hline 10789 & $3.599 \mathrm{E}+09$ & 460549 & $10: 2$ & $p$ & 0.18 .1162 .0 .2 .2 .2 & 1798 & \begin{tabular}{|l|}
6304739 \\
\end{tabular} & 111544834 & 1863731 & 3189634 & 0 & 2077516 & 1668 & 63841 & 0 & 967 & 0 & 967 \\
\hline 10790 & $3.599 \mathrm{E}+09$ & 460549 & $10: 6$ & $p$ & 0.18 .116 .60 .656 .6 & 1798 & 49415032 & 68407317 & 26505403 & 12735002 & 0 & 489824 & 28326 & 36288 & 16167 & 7015 & 0 & 385 \\
\hline 10791 & $3.599 E+09$ & 460549 & $10: 4$ & $P$ & 0.18 .116 .4 .0 .4 .4 & 1798 & 6103717 & 111746125 & 1745707 & 3135503 & 0 & 2085151 & 15092 & 50404 & 8025 & 5624 & 0 & 935 \\
\hline 10792 & $3.599 t+09$ & 460563 & $10: 1$ & $p$ & 0.18 .116 .01 .11 & 1798 & 37476871 & 80371940 & \begin{tabular}{|l|l|}
16505059 \\
\end{tabular} & 12750749 & 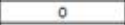 & 1277638 & 20746 & 48529 & 9887 & 7573 & 0 & 1099 \\
\hline 10793 & $3599 E+09$ & 460549 & 10.5 & $P$ & 0.18 .1165 .05 .55 & 1798 & 6499100 & 111349971 & 1978161 & 3258443 & 0 & 2074569 & 5616 & 59837 & 2055 & 2556 & 0 & 1098 \\
\hline 10794 & $3.599 \mathrm{~g}+09$ & 460549 & $10: 3$ & $p$ & 0.18 .1163 .0 .3 .3 .3 & 1798 & 6433759 & 111411377 & \begin{tabular}{|l|}
1997201 \\
\end{tabular} & 2352157 & 0 & 1186162 & 6321 & 59175 & 2677 & 1984 & 0 & 416 \\
\hline
\end{tabular}

Figure 29. Malicious "pwrtrace.csv" $-10,757$ to 10,794 records. 
Malicious "recv.csv": The generated malicious "recv.csv" file consists of 21,573 records and its first 27 records (i.e., 1-27) are depicted below in Figure 30.

\begin{tabular}{|c|c|c|c|c|c|}
\hline B & C & D & E & $\mathrm{F}$ & G \\
\hline No & Real time [us] & $\begin{array}{c}\text { ID } \\
\text { (Receiver) }\end{array}$ & Received message & & $\begin{array}{c}\text { ID } \\
\text { (Sender) }\end{array}$ \\
\hline 1 & 4928647 & ID:1 & DATA recv -> 'Hello' & from & 6 \\
\hline 2 & 5179252 & ID:1 & DATA recv -> 'Hello' & from & 6 \\
\hline 3 & 5427442 & ID:1 & DATA recv -> 'Hello' & from & 6 \\
\hline 4 & 5555622 & ID:1 & DATA recv -> 'Hello' & from & 6 \\
\hline 5 & 5803531 & ID:1 & DATA recv -> 'Hello' & from & 6 \\
\hline 6 & 5926813 & ID:1 & DATA recv -> 'Hello' & from & 6 \\
\hline 7 & 6305514 & ID:1 & DATA recv -> 'Hello' & from & 6 \\
\hline 8 & 6428582 & ID:1 & DATA recv -> 'Hello' & from & 6 \\
\hline 9 & 6677519 & ID:1 & DATA recv -> 'Hello' & from & 6 \\
\hline 10 & 7303554 & ID:1 & DATA recv -> 'Hello' & from & 6 \\
\hline 11 & 8930177 & ID:1 & DATA recv -> 'Hello' & from & 6 \\
\hline 12 & 8939620 & ID:1 & DATA recv -> 'Hello' & from & 6 \\
\hline 13 & 9178554 & ID:1 & DATA recv -> 'Hello' & from & 6 \\
\hline 14 & 9680082 & ID:1 & DATA recv -> 'Hello' & from & 6 \\
\hline 15 & 9690472 & ID:1 & DATA recv -> 'Hello' & from & 6 \\
\hline 16 & 9928890 & ID:1 & DATA recv -> 'Hello' & from & 6 \\
\hline 17 & 9938388 & ID:1 & DATA recv -> 'Hello' & from & 6 \\
\hline 18 & 10178017 & ID:1 & DATA recv -> 'Hello' & from & 6 \\
\hline 19 & 10430699 & ID:1 & DATA recv -> 'Hello' & from & 6 \\
\hline 20 & 10440280 & ID:1 & DATA recv -> 'Hello' & from & 6 \\
\hline 21 & 10680895 & ID:1 & DATA recv -> 'Hello' & from & 6 \\
\hline 22 & 10930537 & ID:1 & DATA recv -> 'Hello' & from & 6 \\
\hline 23 & 11180697 & ID:1 & DATA recv $->$ 'Hello' & from & 6 \\
\hline 24 & 11193252 & ID:1 & DATA recv $->$ 'Hello' & from & 6 \\
\hline 25 & 11796952 & ID:6 & DATA recv 'Reply from server' & & \\
\hline 26 & 11803849 & ID:6 & DATA recv 'Reply from server' & & \\
\hline 27 & 12179677 & ID:1 & DATA recv -> 'Hello' & from & 6 \\
\hline
\end{tabular}

Figure 30. Malicious "recv.csv" -1 to 27 records.

\subsection{Malicious Network Traffic Dataset Generation}

\subsubsection{Malicious Network Traffic Dataset Generation}

The approach followed for the network traffic dataset generation from the UDP flooding attack scenario was similar to the approach followed for the network traffic dataset generation from the benign IIoT network scenario in Section 4.2.1. The "Radio messages" tool, provided by the Cooja simulator, was similarly used for collecting data related to the corresponding network traffic features (e.g., source/destination IPv6 address, packet size, and communication protocol) from the network of the attack scenario. During the simulation, the network traffic information was being shown in the top part of the "Radio messages" output window as depicted in the top part of Figure 31.

When the simulation stopped, the generated pcap file was saved as "radiolog1607519517066.pcap" within the " . . / cooja/build" folder. Afterwards, the "IoT_Simul.sh" file, described in Section 4.2.1, created (a) a new root folder named as "2020-12-09-14-59-59", and (b) the "nettraffic" folder, inside the "2020-12-09-14-59-59" folder, where the "radiolog1607519517066.pcap" file was copied from the "... / cooja/build" folder located in the Cooja Simulator. The "nettraffic" folder inside the root folder "2020-12-09-14-59-59" and the copy of the "radiolog-1607519517066.pcap" file in the "nettraffic" folder are shown in Figure 32. 


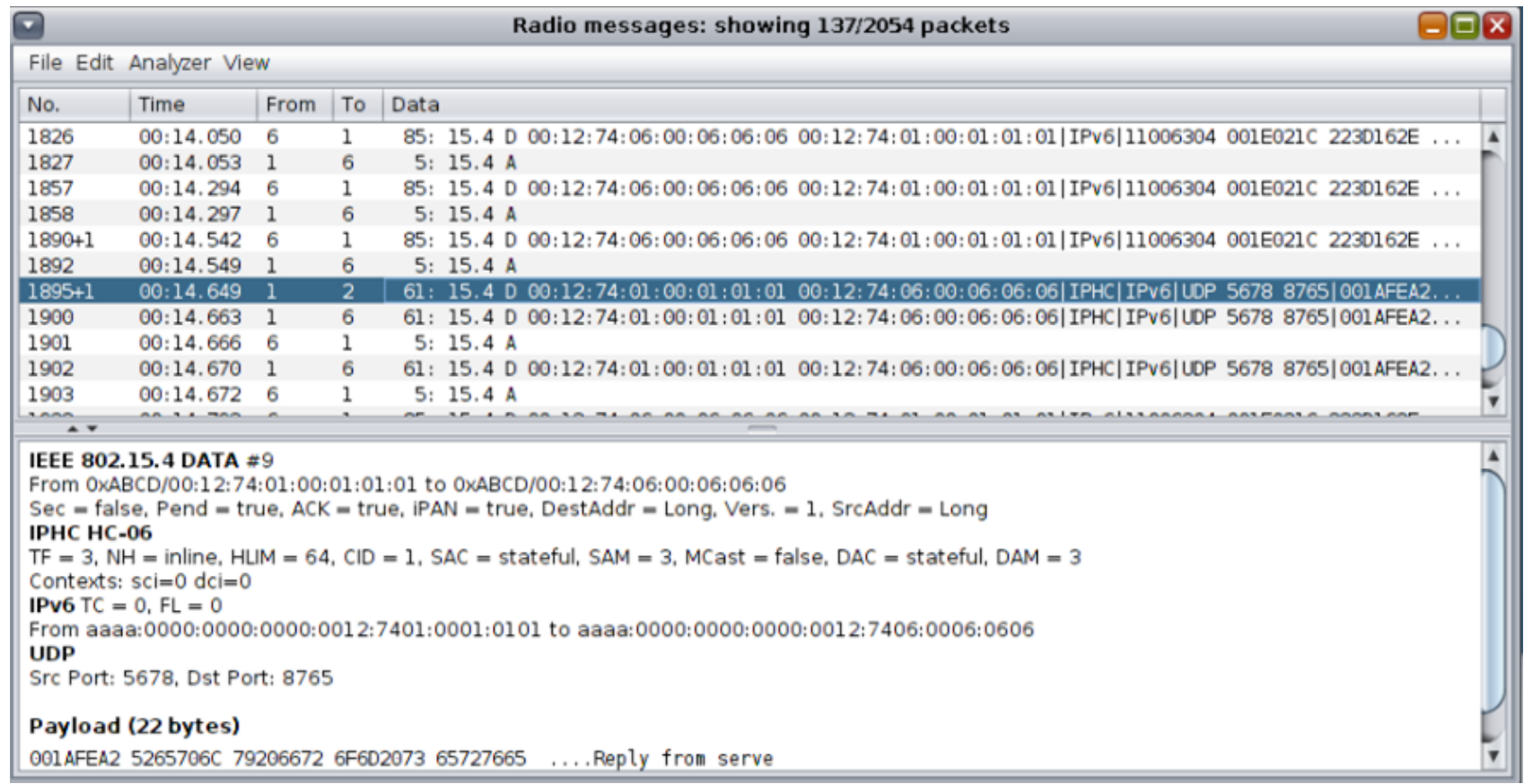

Figure 31. Network traffic information from the attack scenario in the "Radio messages" output window.

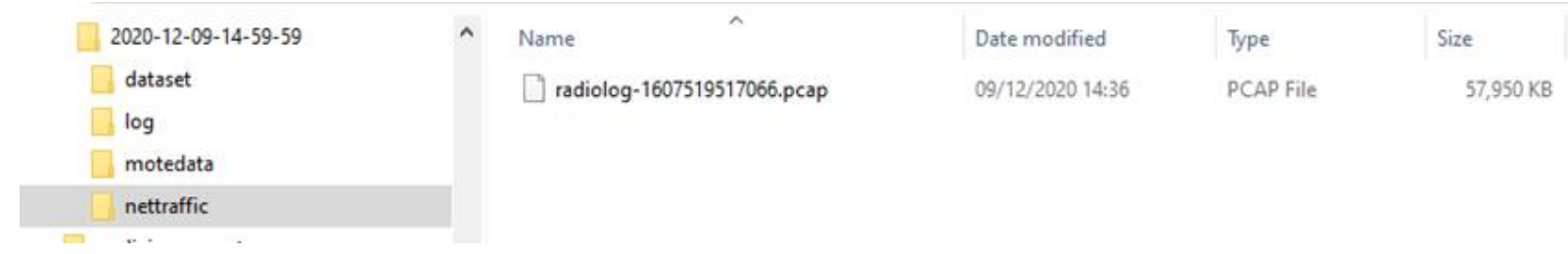

Figure 32. The "nettraffic" folder inside the root folder "2020-12-09-14-59-59" and the copy of the "radiolog1607519517066.pcap" file.

Then, following the same process, as described in Section 4.2.1, we used Wireshark to extract the stored network traffic information from the "radiolog-1607519517066.pcap" file to the "radiolog.csv" file stored in the "nettraffic" folder as shown in Figure 33.

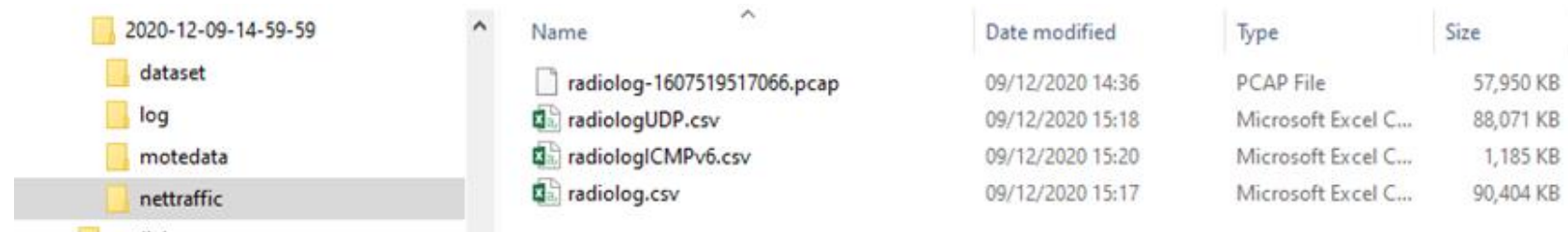

Figure 33. The "nettraffic" folder inside the root folder "2020-12-09-14-59-59" and its included files.

In the "nettraffic" folder, apart from the "radiolog.csv" file, we also used Wireshark, following the same process as in Section 4.2.1, to generate two more files (i.e., the "radiologICMPv6.csv" file and the "radiologUDP.csv" file) from the "radiolog-1607519517066.pcap" file.

\subsubsection{Malicious Network Traffic Datasets-Results}

"radiolog.csv": The generated malicious "radiolog.csv" file consists of 702,332 records and its first 25 records (i.e., 1-25) are depicted below in Figure 34. 


\begin{tabular}{|c|c|c|c|c|c|c|}
\hline B & C & D & E & $\mathrm{F}$ & G & $\mathrm{H}$ \\
\hline No. & Time & Source & Destination & Protocol & Length & Info \\
\hline 1 & 0 & fe80::212:7402:2:202 & ffo2::1a & ICMPv6 & 64 & RPL Control (DODAG Information Solicitation) \\
\hline 2 & 0.032 & fe80::212:7402:2:202 & ff02::1a & ICMPv6 & 64 & RPL Control (DODAG Information Solicitation) \\
\hline 3 & 0.033 & fe80::212:7402:2:202 & ff02::1a & ICMPv6 & 64 & RPL Control (DODAG Information Solicitation) \\
\hline 4 & 0.067 & fe80::212:7402:2:202 & ff02::1a & ICMPv6 & 64 & RPL Control (DODAG Information Solicitation) \\
\hline 5 & 0.1 & fe80::212:7402:2:202 & ff02::1a & ICMPv6 & 64 & RPL Control (DODAG Information Solicitation) \\
\hline 6 & 0.175 & fe80::212:7406:6:606 & ffo2::1a & ICMPv6 & 64 & RPL Control (DODAG Information Solicitation) \\
\hline 7 & 0.176 & fe80::212:7402:2:202 & ffo2::1a & ICMPv6 & 64 & RPL Control (DODAG Information Solicitation) \\
\hline 8 & 0.197 & fe80::212:7406:6:606 & ffo2::1a & ICMPv6 & 64 & RPL Control (DODAG Information Solicitation) \\
\hline 9 & 0.199 & fe80::212:7402:2:202 & ff02::1a & ICMPv6 & 64 & RPL Control (DODAG Information Solicitation) \\
\hline 10 & 0.201 & fe80::212:7406:6:606 & ff02::1a & ICMPv6 & 64 & RPL Control (DODAG Information Solicitation) \\
\hline 11 & 0.203 & fe80::212:7402:2:202 & ff02::1a & ICMPv6 & 64 & RPL Control (DODAG Information Solicitation) \\
\hline 12 & 0.26 & fe80::212:7406:6:606 & ff02::1a & ICMPv6 & 64 & RPL Control (DODAG Information Solicitation) \\
\hline 13 & 0.262 & fe80::212:7402:2:202 & ff02::1a & ICMPv6 & 64 & RPL Control (DODAG Information Solicitation) \\
\hline 14 & 0.329 & fe80::212:7406:6:606 & ff02::1a & ICMPv6 & 64 & RPL Control (DODAG Information Solicitation) \\
\hline 15 & 0.33 & fe80::212:7402:2:202 & ffo2::1a & ICMPv6 & 64 & RPL Control (DODAG Information Solicitation) \\
\hline 16 & 0.332 & fe80::212:7406:6:606 & ffo2::1a & ICMPv6 & 64 & RPL Control (DODAG Information Solicitation) \\
\hline 17 & 0.333 & fe80::212:7402:2:202 & ff02::1a & ICMPv6 & 64 & RPL Control (DODAG Information Solicitation) \\
\hline 18 & 0.391 & fe80::212:7406:6:606 & ff02::1a & ICMPv6 & 64 & RPL Control (DODAG Information Solicitation) \\
\hline 19 & 0.397 & fe80::212:7402:2:202 & ff02::1a & ICMPv6 & 64 & RPL Control (DODAG Information Solicitation) \\
\hline 20 & 0.441 & fe80::212:7406:6:606 & ff02::1a & ICMPv6 & 64 & RPL Control (DODAG Information Solicitation) \\
\hline 21 & 0.459 & fe80::212:7402:2:202 & ff02::1a & ICMPv6 & 64 & RPL Control (DODAG Information Solicitation) \\
\hline 22 & 0.497 & fe80::212:7406:6:606 & ff02::1a & ICMPv6 & 64 & RPL Control (DODAG Information Solicitation) \\
\hline 23 & 0.498 & fe80::212:7402:2:202 & ffo2::1a & ICMPv6 & 64 & RPL Control (DODAG Information Solicitation) \\
\hline 24 & 0.499 & fe80::212:7406:6:606 & ff02::1a & ICMPv6 & 64 & RPL Control (DODAG Information Solicitation) \\
\hline 25 & 0.5 & fe80::212:7402:2:202 & ffo2::1a & ICMPv6 & 64 & RPL Control (DODAG Information Solicitation) \\
\hline
\end{tabular}

Figure 34. Malicious "radiolog.csv" -1 to 25 records.

"radiologICMPv6.csv": The generated malicious "radiologICMPv6.csv" file consists of 9908 records and its first 25 records (i.e., 1-25) are depicted below in Figure 35.

\begin{tabular}{|c|c|c|c|c|c|c|}
\hline B & C & D & E & $\mathrm{F}$ & G & $\mathrm{H}$ \\
\hline No. & Time & Source & Destination & Protocol & Length & Info \\
\hline 1 & 0 & fe $80:: 212: 7402: 2: 202$ & ff02::1a & ICMPv6 & 64 & RPL Control (DODAG Information Solicitation) \\
\hline 2 & 0.032 & fe $80:: 212: 7402: 2: 202$ & ff02::1a & ICMPv6 & 64 & RPL Control (DODAG Information Solicitation) \\
\hline 3 & 0.033 & fe80::212:7402:2:202 & ff02::1a & ICMPv6 & 64 & RPL Control (DODAG Information Solicitation) \\
\hline 4 & 0.067 & fe $80:: 212: 7402: 2: 202$ & ff02::1a & ICMPv6 & 64 & RPL Control (DODAG Information Solicitation) \\
\hline 5 & 0.1 & fe80::212:7402:2:202 & ff02::1a & ICMPv6 & 64 & RPL Control (DODAG Information Solicitation) \\
\hline 6 & 0.175 & fe80::212:7406:6:606 & ff02::1a & ICMPv6 & 64 & RPL Control (DODAG Information Solicitation) \\
\hline 7 & 0.176 & fe80::212:7402:2:202 & ff02::1a & ICMPv6 & 64 & RPL Control (DODAG Information Solicitation) \\
\hline 8 & 0.197 & fe80::212:7406:6:606 & ff02::1a & ICMPv6 & 64 & RPL Control (DODAG Information Solicitation) \\
\hline 9 & 0.199 & fe80::212:7402:2:202 & ff02::1a & ICMPv6 & 64 & RPL Control (DODAG Information Solicitation) \\
\hline 10 & 0.201 & fe80::212:7406:6:606 & ff02::1a & ICMPv6 & 64 & RPL Control (DODAG Information Solicitation) \\
\hline 11 & 0.203 & fe80::212:7402:2:202 & ff02::1a & ICMPv6 & 64 & RPL Control (DODAG Information Solicitation) \\
\hline 12 & 0.26 & fe80::212:7406:6:606 & ff02::1a & ICMPv6 & 64 & RPL Control (DODAG Information Solicitation) \\
\hline 13 & 0.262 & fe80::212:7402:2:202 & ff02::1a & ICMPv6 & 64 & RPL Control (DODAG Information Solicitation) \\
\hline 14 & 0.329 & fe80::212:7406:6:606 & ff02::1a & ICMPv6 & 64 & RPL Control (DODAG Information Solicitation) \\
\hline 15 & 0.33 & fe80::212:7402:2:202 & ff02::1a & ICMPv6 & 64 & RPL Control (DODAG Information Solicitation) \\
\hline 16 & 0.332 & fe80::212:7406:6:606 & ff02::1a & ICMPv6 & 64 & RPL Control (DODAG Information Solicitation) \\
\hline 17 & 0.333 & fe80::212:7402:2:202 & $f f 02:: 1 a$ & ICMPv6 & 64 & RPL Control (DODAG Information Solicitation) \\
\hline 18 & 0.391 & fe80::212:7406:6:606 & ff02::1a & ICMPv6 & 64 & RPL Control (DODAG Information Solicitation) \\
\hline 19 & 0.397 & fe80::212:7402:2:202 & ff02::1a & ICMPv6 & 64 & RPL Control (DODAG Information Solicitation) \\
\hline 20 & 0.441 & fe80::212:7406:6:606 & ff02::1a & ICMPv6 & 64 & RPL Control (DODAG Information Solicitation) \\
\hline 21 & 0.459 & feso::212:7402:2:202 & ff02::1a & ICMPv6 & 64 & RPL Control (DODAG Information Solicitation) \\
\hline 22 & 0.497 & fe80::212:7406:6:606 & ff02::1a & ICMPv6 & 64 & RPL Control (DODAG Information Solicitation) \\
\hline 23 & 0.498 & fe80::212:7402:2:202 & $f f 02:: 1 a$ & ICMPv6 & 64 & RPL Control (DODAG Information Solicitation) \\
\hline 24 & 0.499 & fe80::212:7406:6:606 & ff02::1a & ICMPv6 & 64 & RPL Control (DODAG Information Solicitation) \\
\hline 25 & 0.5 & fe80::212:7402:2:202 & ff02::1a & ICMPv6 & 64 & RPL Control (DODAG Information Solicitation) \\
\hline
\end{tabular}

Figure 35. Malicious "radiologICMPv6.csv" -1 to 25 records. 
"radiologUDP.csv": The generated malicious "radiologUDP.csv" file consists of 670,671 records and its first 25 records (i.e., 1-25) are depicted below in Figure 36.

\begin{tabular}{|c|c|c|c|c|c|c|}
\hline B & C & D & E & $\mathrm{F}$ & G & $\mathrm{H}$ \\
\hline No. & Time & Source & Destination & Protocol & Length & Info \\
\hline 1 & 1.234 & aaaa::212:7406:6:606 & aaaa::ff:fe00:1 & UDP & 85 & Source port: ultraseek-http Destination port: rrac \\
\hline 2 & 1.235 & aаaа::212:7406:6:606 & aaaa::ff:fe00:1 & UDP & 85 & Source port: ultraseek-http Destination port: rrac \\
\hline 3 & 1.236 & aaaa::212:7406:6:606 & aaaa::ff:fe00:1 & UDP & 85 & Source port: ultraseek-http Destination port: rrac \\
\hline 4 & 1.236 & aaaa::212:7406:6:606 & aaaa::ff:fe00:1 & UDP & 85 & Source port: ultraseek-http Destination port: rrac \\
\hline 5 & 1.237 & aaaa::212:7406:6:606 & aaaa::ff:fe00:1 & UDP & 85 & Source port: ultraseek-http Destination port: rrac \\
\hline 6 & 1.238 & aaaa::212:7406:6:606 & aaaa::ff:fe00:1 & UDP & 85 & Source port: ultraseek-http Destination port: rrac \\
\hline 7 & 1.239 & aaaa::212:7406:6:606 & aaaa::ff:fe00:1 & UDP & 85 & Source port: ultraseek-http Destination port: rrac \\
\hline 8 & 1.24 & aaaa::212:7406:6:606 & aaaa::ff:fe00:1 & UDP & 85 & Source port: ultraseek-http Destination port: rrac \\
\hline 9 & 1.24 & aaaa::212:7406:6:606 & aaaa::ff:fe00:1 & UDP & 85 & Source port: ultraseek-http Destination port: rrac \\
\hline 10 & 1.241 & aaaa::212:7406:6:606 & aaaa::ff:fe00:1 & UDP & 85 & Source port: ultraseek-http Destination port: rrac \\
\hline 11 & 1.242 & aaaa::212:7406:6:606 & aaaa::ff:fe00:1 & UDP & 85 & Source port: ultraseek-http Destination port: rrac \\
\hline 12 & 1.242 & aaaa::212:7406:6:606 & aaaa::ff:fe00:1 & UDP & 85 & Source port: ultraseek-http Destination port: rrac \\
\hline 13 & 1.243 & aaaa::212:7406:6:606 & aaaa::ff:fe00:1 & UDP & 85 & Source port: ultraseek-http Destination port: rrac \\
\hline 14 & 1.243 & aaaa::212:7406:6:606 & aaaa::ff:fe00:1 & UDP & 85 & Source port: ultraseek-http Destination port: rrac \\
\hline 15 & 1.244 & aaaa::212:7406:6:606 & aaaa::ff:fe00:1 & UDP & 85 & Source port: ultraseek-http Destination port: rrac \\
\hline 16 & 1.245 & aaaa::212:7406:6:606 & aaaa::ff:fe00:1 & UDP & 85 & Source port: ultraseek-http Destination port: rrac \\
\hline 17 & 1.245 & aaaa::212:7406:6:606 & aaaa::ff:fe00:1 & UDP & 85 & Source port: ultraseek-http Destination port: rrac \\
\hline 18 & 1.246 & aaaa::212:7406:6:606 & aaaa::ff:fe00:1 & UDP & 85 & Source port: ultraseek-http Destination port: rrac \\
\hline 19 & 1.246 & aaaa::212:7406:6:606 & aaaa::ff:fe00:1 & UDP & 85 & Source port: ultraseek-http Destination port: rrac \\
\hline 20 & 1.247 & aaaa::212:7406:6:606 & aaaa::ff:fe00:1 & UDP & 85 & Source port: ultraseek-http Destination port: rrac \\
\hline 21 & 1.248 & aaaa::212:7406:6:606 & aaaa::ff:fe00:1 & UDP & 85 & Source port: ultraseek-http Destination port: rrac \\
\hline 22 & 1.248 & aaaa::212:7406:6:606 & aaaa::ff:fe00:1 & UDP & 85 & Source port: ultraseek-http Destination port: rrac \\
\hline 23 & 1.249 & aaaa::212:7406:6:606 & aaaa::ff:fe00:1 & UDP & 85 & Source port: ultraseek-http Destination port: rrac \\
\hline 24 & 1.25 & aaaa::212:7406:6:606 & aaaa::ff:fe00:1 & UDP & 85 & Source port: ultraseek-http Destination port: rrac \\
\hline 25 & 1.25 & aaaa::212:7406:6:606 & aaaa::ff:fe00:1 & UDP & 85 & Source port: ultraseek-http Destination port: rrac \\
\hline
\end{tabular}

Figure 36. Malicious "radiologUDP.csv" -1 to 25 records.

\section{Discussion on the Generated Datasets}

The generated benign and malicious "pwrtrace" datasets, presented in Sections 4.1.2 and 5.1.2, respectively, include information about raw features (e.g., all_cpu, all_lpm, all_transmit, all_listen) which can be used to derive new features more informative, in terms of the behaviour of each mote, and non-redundant. These new features are intended to constitute valuable features for training and evaluating AIDS for IoT/IIoT networks. Towards this direction, the total energy consumption of a mote in an IoT/IIoT network can be considered as a valuable feature for detection of a UDP flooding attack and its source as the compromised mote carrying out the attack is characterised by high total energy consumption, as demonstrated below.

Based on $[29,30]$, the total energy consumption of each mote, at the reading (i.e., record) $i$, is given by the sum of (a) the energy consumption in the CPU state; (b) the energy consumption in the LPM state; (c) the energy consumption in the Tx state; and the average power consumption Listen state, at the reading (i.e., record) $i$, as shown in the equation below:

$$
\begin{aligned}
\mathrm{E}_{\text {total }_{\mathrm{i}}}(\mathrm{mj}) & =\mathrm{E}_{\mathrm{cpu}_{\mathrm{total}_{\mathrm{i}}}}+\mathrm{E}_{\mathrm{lpm}_{\mathrm{total}_{\mathrm{i}}}}+\mathrm{E}_{\mathrm{tx}_{\mathrm{total}_{\mathrm{i}}}}+\mathrm{E}_{\mathrm{rx}_{\mathrm{total}_{\mathrm{i}}}}= \\
& =\left(\mathrm{I}_{\mathrm{cpu}} \times \mathrm{V}_{\mathrm{cpu}} \times \mathrm{T}_{\mathrm{cpu}_{\mathrm{i}}}\right)+\left(\mathrm{I}_{\mathrm{lpm}} \times \mathrm{V}_{\mathrm{lpm}} \times \mathrm{T}_{\mathrm{lpm}_{\mathrm{i}}}\right)+\left(\mathrm{I}_{\mathrm{tx}} \times \mathrm{V}_{\mathrm{tx}} \times \mathrm{T}_{\mathrm{tx}_{\mathrm{i}}}\right)+\left(\mathrm{I}_{\mathrm{rx}} \times \mathrm{V}_{\mathrm{rx}} \times \mathrm{T}_{\mathrm{rx}_{\mathrm{i}}}\right)
\end{aligned}
$$

where

$\mathrm{I}_{\mathrm{cpu}}$ : the nominal current in the CPU state;

$\mathrm{I}_{\mathrm{lpm}}$ : the nominal current in the LPM state;

$\mathrm{I}_{\mathrm{tx}}$ : the nominal current in the TX state;

$\mathrm{I}_{\mathrm{rx}}$ : the nominal current in the RX state;

$\mathrm{V}_{\mathrm{cpu}}$ : the nominal voltage in the CPU state; 
$\mathrm{V}_{\mathrm{lpm}}$ : the nominal voltage in the LPM state;

$\mathrm{V}_{\mathrm{tx}}$ : the nominal voltage in the TX state;

$\mathrm{V}_{\mathrm{rx}}$ : the nominal voltage in the RX state;

$T_{c p u_{i}}=\frac{c p u_{i}(\# \text { ticks })}{\text { RTIMER_ARCH_SECOND }}=\frac{c p u_{i}(\# \text { ticks })}{32,768}$
$T_{l p m_{i}}=\frac{l p m_{i}(\# \text { ticks })}{\text { RTIMER_ARCH_SECOND }}=\frac{\text { lpm } m_{i}(\# \text { ticks })}{32,768}$
$T_{t x_{i}}=\frac{t x_{i}(\# \text { ticks })}{\text { RTIMER_ARCH_SECOND }}=\frac{t x_{i}(\# \text { ticks })}{32,768}$
$T_{r x_{i}}=\frac{r x_{i}(\# \text { ticks })}{\text { RTIMER_ARCH_SECOND }}=\frac{r x_{i}(\# \text { ticks })}{32,768}$

Based on Equation (1) and Table 2 that provides the typical operating conditions for a Tmote Sky mote, the total energy consumption, at the reading (i.e., record) i, is given by Equation (2):

$$
\begin{aligned}
\mathrm{E}_{\text {total }_{\mathrm{i}}(\mathrm{mj})=} & 1.8 \times 3 \times\left(\frac{\mathrm{cpu}_{\mathrm{i}}(\# \text { ticks })}{32,768}\right) \\
& +0.0545 \times 3 \times\left(\frac{\mathrm{lpm}_{\mathrm{i}}(\# \text { ticks })}{32,768}\right) \\
& +19.5 \times 3 \times\left(\frac{\mathrm{tx}_{\mathrm{i}}(\# \text { ticks })}{32,768}\right) \\
& +21.8 \times 3 \times\left(\frac{\mathrm{rx}_{\mathrm{i}}(\# \text { ticks })}{32,768}\right)
\end{aligned}
$$

\begin{tabular}{|c|c|c|c|c|}
\hline & MIN & NOM (Typical) & MAX & UNIT \\
\hline Supply voltage & 2.1 & 3.0 & 3.6 & $\mathrm{~V}$ \\
\hline Supply voltage during flash memory programming & 2.7 & 3.0 & 3.6 & $\mathrm{~V}$ \\
\hline Operating free air temperature & -40 & & 85 & ${ }^{\circ} \mathrm{C}$ \\
\hline Current Consumption: MCU on, Radio RX & & 21.8 & 23 & $\mathrm{~mA}$ \\
\hline Current Consumption: MCU on, Radio TX & & 19.5 & 21 & $\mathrm{~mA}$ \\
\hline Current Consumption: MCU on, Radio off & & 1800 & 2400 & $\mu \mathrm{A}$ \\
\hline Current Consumption: MCU idle, Radio off & & 54.5 & 1200 & $\mu \mathrm{A}$ \\
\hline Current Consumption: MCU standby & & 5.1 & 21.0 & $\mu \mathrm{A}$ \\
\hline
\end{tabular}

Table 2. Typical Operating Conditions for Tmote Sky motes.

Based on Equation (2) and the following features, from the generated benign "powertrace" dataset, for each mote: (a) all_cpu; (b) all_lpm; (c) all_transmit; and (d) all_listen, the total energy consumption by each mote, during the simulation time (i.e., $60 \mathrm{~min}=3600 \mathrm{~s}$ ) is shown below in Figure 37.

On the other hand, based on Equation (2) and the same features (i.e., all_cpu, all_lpm, all_transmit; and all_listen) for each mote, from the generated malicious "powertrace" dataset, the total energy consumption by each mote, during the simulation time (i.e., $60 \mathrm{~min}=3600 \mathrm{~s}$ ) is shown below.

As shown in Figure 38, mote6, which is the compromised client that carried out the UDP flooding attack, consumed much more energy than any other legitimate client and the legitimate server in the UDP flooding attack scenario. Moreover, mote6 in the UDP flooding attack consumed much more energy than the energy it consumed in the benign scenario as demonstrated in Figure 37.

Furthermore, the generated benign and malicious network traffic datasets, presented in Sections 4.2.2 and 5.2.2, respectively, include information about raw features, such as source/destination address, protocol, which can be used to derive new features more informative, in terms of the behaviour of the network traffic, and non-redundant. These new features are also intended to constitute valuable features for training and evaluating AIDS for IoT/IIoT networks. From the network traffic point of view, the total RPL (Routing Protocol for Low-Power and Lossy Networks) messages overhead of the IoT/IIoT network can be considered as a feature for detection of a UDP flooding attack as an IoT/IIoT network 
under a UDP flooding attack is characterised by low total RPL messages overhead because of the huge amount of the UDP messages flooding the network, as shown below.

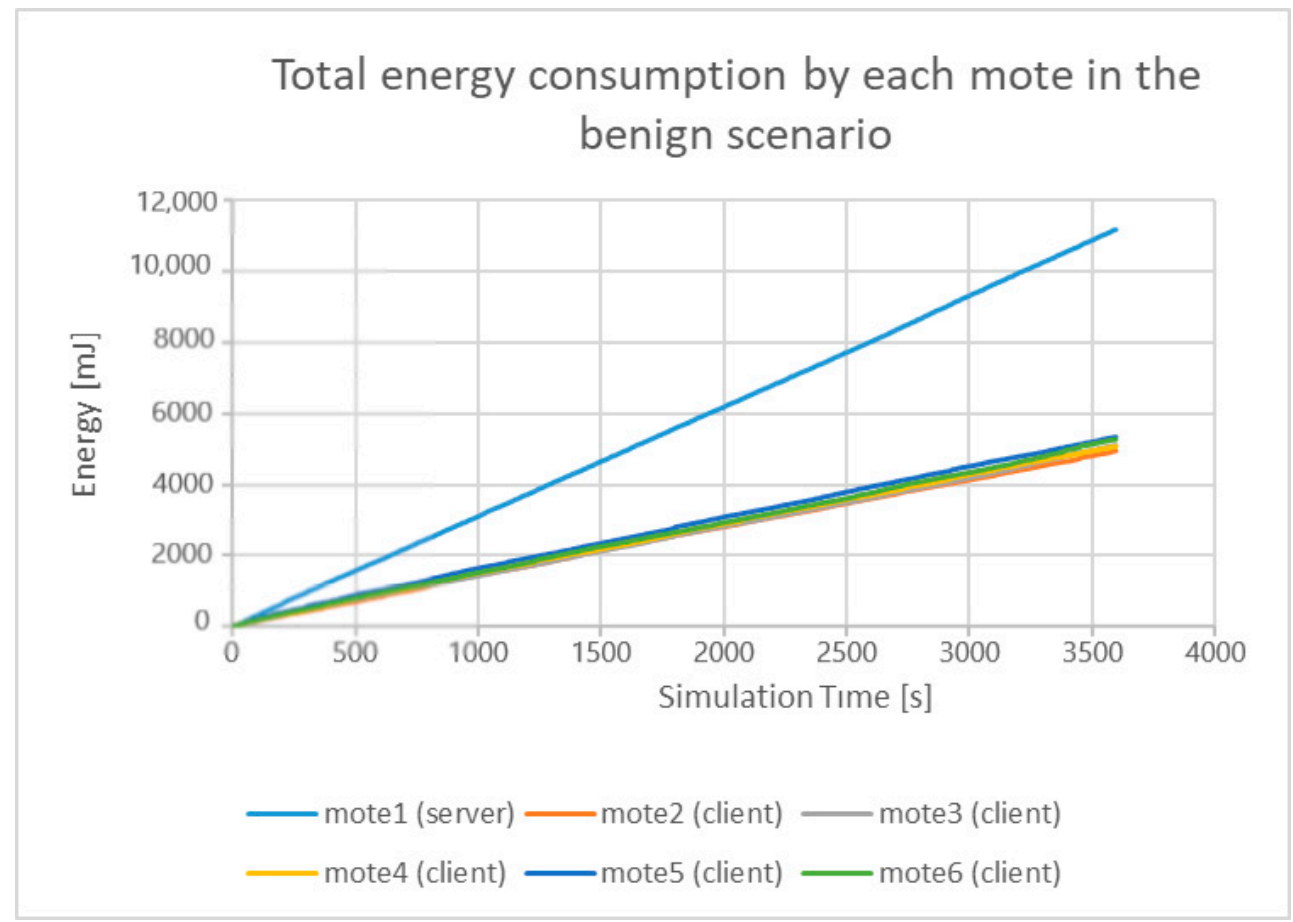

Figure 37. Total energy consumption by each mote in the benign scenario.

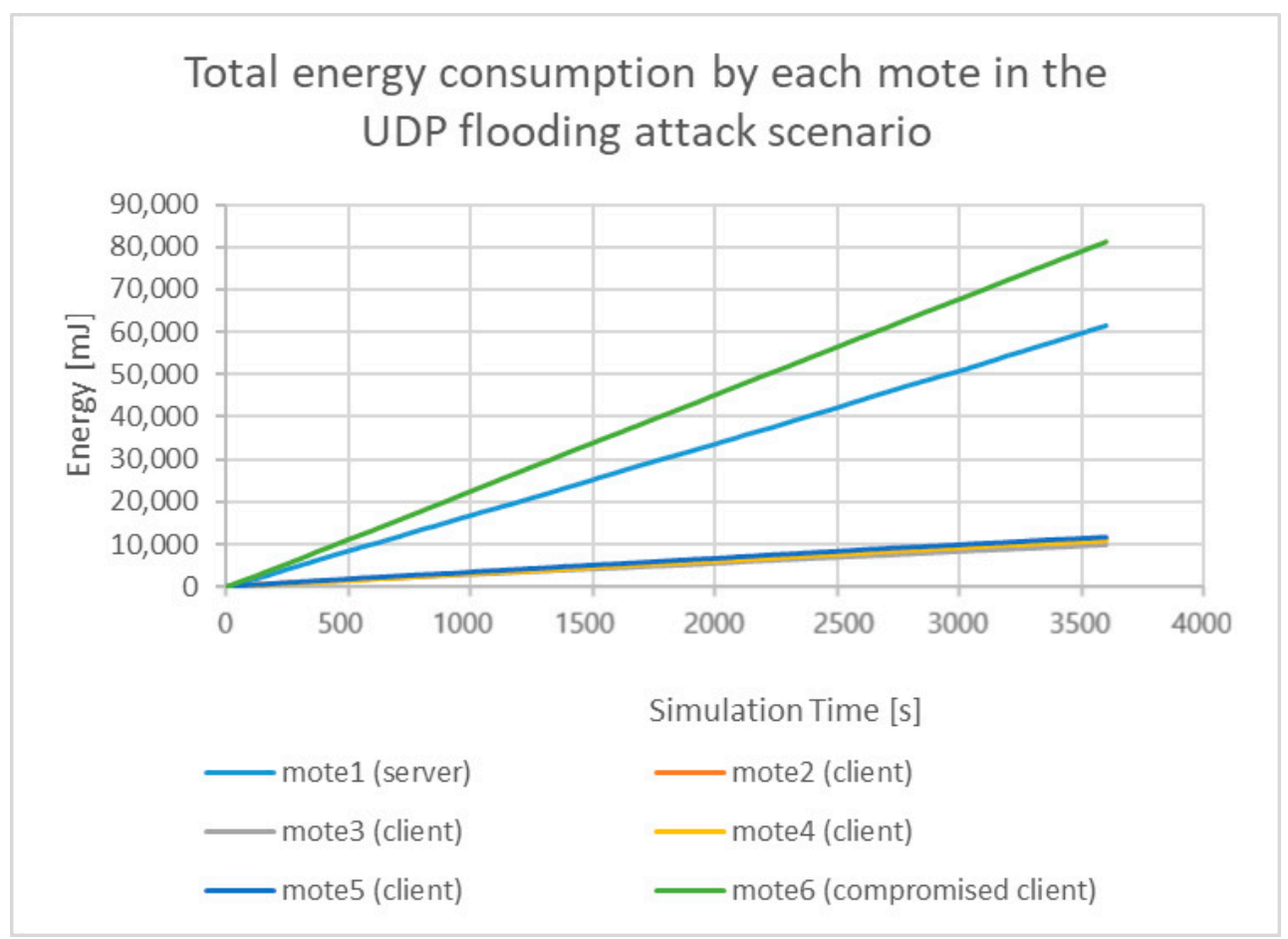

Figure 38. Total energy consumption by each mote in the UDP flooding attack scenario.

Table 3 was extracted from the benign network traffic dataset (i.e., benign "radiolog.csv") and shows, in the last column, the percentage of the RPL messages overhead per mote which is calculated as follows: the number of RPL messages per mote over the total number of exchanged messages within the network during the simulation time (i.e., 
116,463 messages). The last row of Table 3 contains the total number of RPL messages (7975), UDP messages $(104,048)$, and other protocol messages (4440) exchanged within the network, and the total RPL messages overhead (\%).

Table 3. RPL messages overhead of the IoT/IIoT network in the benign scenario.

\begin{tabular}{|ccccc|}
\hline & $\begin{array}{c}\text { Number of RPL } \\
\text { Messages }\end{array}$ & $\begin{array}{c}\text { Number of } \\
\text { UDP Messages }\end{array}$ & $\begin{array}{c}\text { Number of } \\
\text { Other Messages }\end{array}$ & $\begin{array}{c}\text { RPL Overhead } \\
\text { (\%) }\end{array}$ \\
\hline Mote 1 & 290 & 43,804 & N/A & 0.25 \\
Mote 2 & 1982 & 11,621 & N/A & 1.70 \\
\hline Mote 3 & 1621 & 11,883 & N/A & 1.39 \\
Mote 4 & 1604 & 11,827 & N/A & 1.38 \\
\hline Mote 5 & 1308 & 12,556 & N/A & 1.12 \\
\hline Mote 6 & 1170 & 12,357 & N/A & 1.00 \\
\hline Total & 7975 & 104,048 & 4440 & 6.85 \\
\hline
\end{tabular}

Based on the information included in Table 3, the calculated RPL messages overhead per mote and the total RPL messages overhead are depicted in Figure 39.

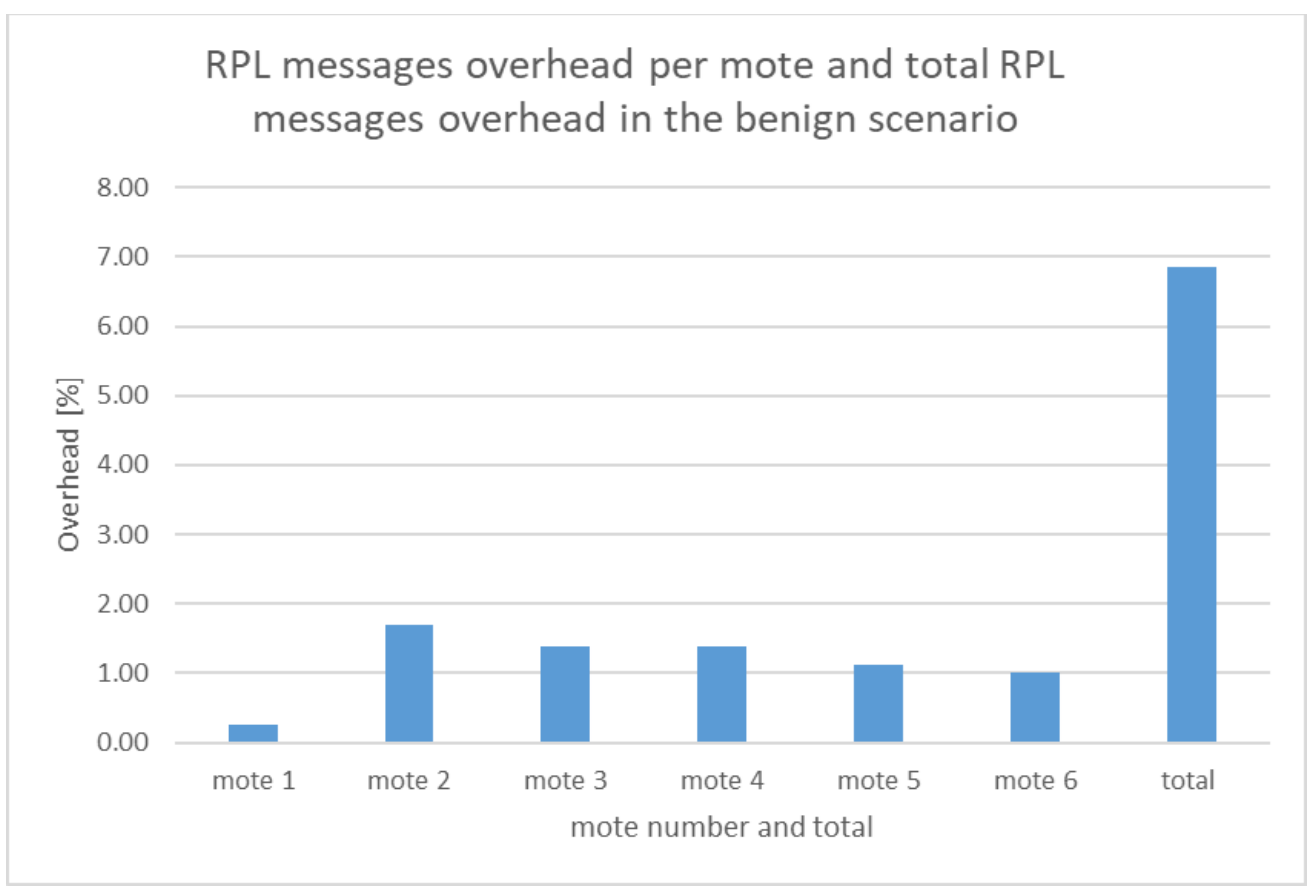

Figure 39. RPL messages overhead per mote and total RPL messages overhead in the benign scenario.

On the other hand, Table 4 was extracted from the malicious network traffic dataset (i.e., malicious "radiolog.csv") reflecting the UDP flooding attack scenario. Similar to Table 3, Table 4 shows, in the last column, the percentage of the RPL messages overhead per mote which is calculated as follows: the number of RPL messages per mote over the total number of exchanged messages within the network during the simulation time (i.e., 702,332 messages). The last row of Table 4 contains the total number of RPL messages (9908), UDP messages $(670,671)$, and other protocol messages $(21,753)$ exchanged within the network, and the total RPL messages overhead (\%). 
Table 4. RPL messages overhead of the IoT/IIoT network in the benign scenario.

\begin{tabular}{|ccccc|}
\hline & $\begin{array}{c}\text { Number of RPL } \\
\text { Messages }\end{array}$ & $\begin{array}{c}\text { Number of } \\
\text { UDP Messages }\end{array}$ & $\begin{array}{c}\text { Number of } \\
\text { Other Messages }\end{array}$ & $\begin{array}{c}\text { RPL Overhead } \\
(\%)\end{array}$ \\
\hline Mote 1 & 203 & 254,796 & N/A & 0.03 \\
Mote 2 & 2228 & 28,953 & N/A & 0.32 \\
\hline Mote 3 & 2768 & 30,238 & N/A & 0.39 \\
Mote 4 & 1976 & 27,260 & N/A & 0.28 \\
\hline Mote 5 & 2084 & 31,247 & N/A & 0.30 \\
\hline Mote 6 & 6490 & 298,177 & N/A & 0.09 \\
\hline Total & 9908 & 670,671 & 21,753 & 1.41 \\
\hline
\end{tabular}

Based on the information included in Table 4, the calculated RPL messages overhead per mote and the total RPL messages overhead are depicted in Figure 40.

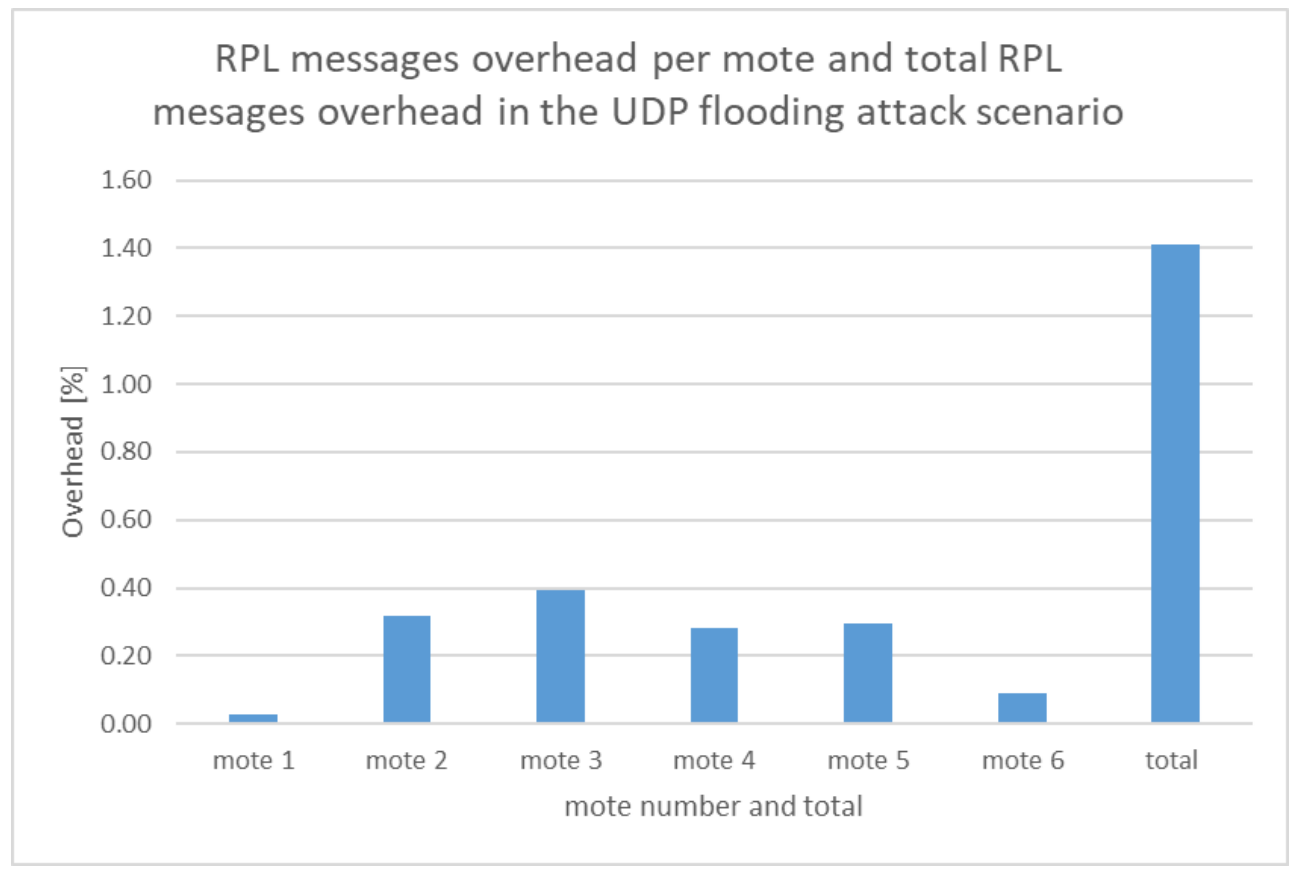

Figure 40. RPL messages overhead per mote and total RPL messages overhead in the malicious scenario.

As shown in Figures 39 and 40, the total RPL messages overhead (1.41\%) in the malicious scenario is much less than the total RPL messages overhead in the benign scenario $(6.85 \%)$ because of the huge amount of the UDP messages flooding the network in the malicious scenario.

\section{Conclusions}

Due to the urgent need for up-to-date, representative and well-structured IoT/IIoTspecific datasets which are publicly available and constitute benchmark datasets for training and evaluating ML models used in AIDSs for IoT/IIoT networks, we target the generation of new labelled IoT/IIoT datasets that will be publicly available to the research community and include (i) events reflecting multiple benign and attack scenarios from current IoT/IIoT network environments, (ii) sensor measurement data, (iii) network-related information (e.g., packet-level information and flow-level information) from the IoT/IIoT network, and (iv) information related to the behaviour of the IoT/IIoT devices deployed within the IoT/IIoT network. In this context, this paper we presented an initial set of datasets with these significant characteristics for effective training and testing of ML models used in AIDSs for protecting IoT/IIoT networks. In particular, the provided set of datasets consists of (a) benign IoT/IIoT datasets (i.e., around 11,000 records of the benign "powertrace" dataset and around 116,000 records of the benign network traffic dataset), and (b) malicious 
IoT/IIoT datasets (i.e., around 11,000 records of the malicious "powertrace" dataset and around 700,000 records of the malicious network traffic dataset).

In addition, in this paper, we presented in detail the approach that we adopted to generate the initial set of benign IoT/IIoT and malicious IoT/IIoT datasets by utilising the Cooja simulator that was the simulation environment where the corresponding benign and attack scenarios were implemented. It is worthwhile to highlight that for the first time and to the best of our knowledge, that the Cooja simulator, which is the companion network simulator of Contiki OS (one of the most popular OSs for resource constrained IoT devices), was used in a systematic way in order to generate IoT/IIoT datasets. In particular, we provided a comprehensive description of the whole approach we followed in order to acquire the generated datasets within csv files from the captured raw information residing in the Cooja simulator environment. Then, the generated datasets in csv format are ready to feed ML algorithms for training and testing purposes.

Our goal is that the new labelled IoT/IIoT datasets generated by utilizing the Cooja simulator should not to be considered as a replacement of datasets captured from real IoT/IIoT networks or real IoT/IIoT testbeds, but instead to be considered as complementary datasets that will contribute to fill the gap in the lack of publicly available up-to-date, representative and well-structured IoT/IIoT-specific datasets that constitute benchmark datasets for training and evaluating ML models used in AIDSs for IoT/IIoT networks.

As future work, we plan to continue working on the implementation of more benign IoT/IIoT network scenarios and various types of IoT/IIoT network attack scenarios, with more motes, in Cooja simulator in order to generate richer benign and malicious datasets for more effective training and testing of ML algorithms used in AIDSs for protecting IoT/IIoT networks such as the one described in [31]. Our intention is to make the generated rich datasets publicly available to the research community. In addition, we will also make publicly available the Cooja-based framework that will have been developed in order to generate the rich datasets. This will allow researchers to reproduce datasets as well as generate new datasets for their own scenarios without having to "reinvent the wheel". Furthermore, we intend to analyse the generated datasets to select the most appropriate features for accurate and efficient detection of different types of attacks within an IoT/IIoT network. Finally, we plan to apply a number of common ML algorithms (e.g., support vector machines (SVMs), Naïve Bayes, k-nearest neighbour, logistics regression, etc.) to evaluate their performance on the new generated datasets when these algorithms are used for anomaly detection in AIDSs.

Author Contributions: Conceptualization and methodology, G.M., J.C.R., and I.E.; software, J.C.R. and I.E.; validation, J.C.R. and G.M.; investigation, I.E., J.C.R., and G.M.; resources, I.E., J.C.R., and M.P.; writing—original draft preparation, I.E., M.P., and G.Z.; writing-review and editing, I.E., G.M., and J.R.; visualization, J.C.R. and I.E.; supervision, G.M. and J.R. All authors have read and agreed to the published version of the manuscript.

Funding: This research received external funding.

Institutional Review Board Statement: Not applicable.

Informed Consent Statement: Not applicable.

Acknowledgments: The research work leading to this publication has received funding through the Moore4Medical project under grant agreement H2020-ECSEL-2019-IA-876190 within ECSEL JU in collaboration with the European Union's H2020 Framework Programme (H2020/2014-2020) and Fundação para a Ciência e Tecnologia (ECSEL/0006/2019).

Conflicts of Interest: The authors declare no conflict of interest.

\section{References}

1. Xu, L.D.; He, W.; Li, S. Internet of Things in Industries: A Survey. IEEE Trans. Ind. Informatics 2014, 10, 2233-2243. [CrossRef]

2. Zarpelão, B.B.; Miani, R.S.; Kawakani, C.T.; de Alvarenga, S.C. A survey of intrusion detection in Internet of Things. J. Netw. Comput. Appl. 2017, 84, 25-37. [CrossRef] 
3. Sisinni, E.; Saifullah, A.; Han, S.; Jennehag, U.; Gidlund, M. Industrial Internet of Things: Challenges, Opportunities, and Directions. IEEE Trans. Ind. Informatics 2018, 14, 4724-4734. [CrossRef]

4. Alsaedi, A.; Moustafa, N.; Tari, Z.; Mahmood, A.; Anwar, A. TON_IoT Telemetry Dataset: A New Generation Dataset of IoT and IIoT for Data-Driven Intrusion Detection Systems. IEEE Access 2020, 8, 165130-165150. [CrossRef]

5. Chaabouni, N.; Mosbah, M.; Zemmari, A.; Sauvignac, C.; Faruki, P. Network Intrusion Detection for IoT Security Based on Learning Techniques. IEEE Commun. Surv. Tutorials 2019, 21, 2671-2701. [CrossRef]

6. KDD Cup 1999 Data. Available online: http://kdd.ics.uci.edu/databases/kddcup99/kddcup99.html (accessed on 19 September 2020).

7. Tavallaee, M.; Bagheri, E.; Lu, W.; Ghorbani, A.A. A detailed analysis of the KDD CUP 99 data set. In Proceedings of the IEEE Symposium on Computational Intelligence for Security and Defense Applications, CISDA 2009, Ottawa, ON, Canada, 8-10 July 2009; pp. 1-6.

8. Moustafa, N.; Slay, J. UNSW-NB15: A comprehensive data set for network intrusion detection systems (UNSW-NB15 network data set). In Proceedings of the 2015 Military Communications and Information Systems Conference, MilCIS 2015, Canberra, ACT, Australia, 10-12 November 2015; pp. 1-6.

9. Sharafaldin, I.; Lashkari, A.H.; Ghorbani, A.A. Toward Generating a New Intrusion Detection Dataset and Intrusion Traffic Characterization. In Proceedings of the ICISSP2018, Funchal, Madeira, Portugal, 22-24 January 2018; pp. 108-116.

10. Suthaharan, S.; Alzahrani, M.; Rajasegarar, S.; Leckie, C.; Palaniswami, M. Labelled data collection for anomaly detection in wireless sensor networks. In Proceedings of the 2010 6th International Conference on Intelligent Sensors, Sensor Networks and Information Processing, ISSNIP 2010, Brisbane, QLD, Australia, 7-10 December 2010; pp. 269-274.

11. Sivanathan, A.; Gharakheili, H.H.; Loi, F.; Radford, A.; Wijenayake, C.; Vishwanath, A.; Sivaraman, V. Classifying IoT Devices in Smart Environments Using Network Traffic Characteristics. IEEE Trans. Mob. Comput. 2019, 18, 1745-1759. [CrossRef]

12. Koroniotis, N.; Moustafa, N.; Sitnikova, E.; Turnbull, B. Towards the development of realistic botnet dataset in the Internet of Things for network forensic analytics: Bot-IoT dataset. Futur. Gener. Comput. Syst. 2019, 100, 779-796. [CrossRef]

13. Hamza, A.; Gharakheili, H.H.; Benson, T.A.; Sivaraman, V. Detecting Volumetric Attacks on IoT Devices via SDN-Based Monitoring of MUD Activity. In Proceedings of the SOSR 2019-Proceedings of the 2019 ACM Symposium on SDN Research, San Jose, CA, USA, 3-4 April 2019; Association for Computing Machinery, Inc: New York, NY, USA, 2019 ; pp. 36-48.

14. Österlind, F.; Dunkels, A.; Eriksson, J.; Finne, N.; Voigt, T. Cross-level sensor network simulation with COOJA. In Proceedings of the Proceedings-Conference on Local Computer Networks, LCN, Tampa, FL, USA, 14-16 November 2006; pp. 641-648.

15. ITU-T. Recommendation ITU-T Y.2060 “Overview of the Internet of Things". 2012. Available online: https:/ / www.itu.int/ITU$\mathrm{T} /$ recommendations / rec.aspx?rec=y.2060 (accessed on 15 December 2020).

16. Qi, Q.; Tao, F. A Smart Manufacturing Service System Based on Edge Computing, Fog Computing, and Cloud Computing. IEEE Access 2019, 7, 86769-86777. [CrossRef]

17. Lin, J.; Yu, W.; Zhang, N.; Yang, X.; Zhang, H.; Zhao, W. A Survey on Internet of Things: Architecture, Enabling Technologies, Security and Privacy, and Applications. IEEE Internet Things J. 2017, 4, 1125-1142. [CrossRef]

18. Ferrag, M.A.; Maglaras, L.; Argyriou, A.; Kosmanos, D.; Janicke, H. Security for 4G and 5G cellular networks: A survey of existing authentication and privacy-preserving schemes. J. Netw. Comput. Appl. 2018, 101, 55-82. [CrossRef]

19. Makhdoom, I.; Abolhasan, M.; Lipman, J.; Liu, R.P.; Ni, W. Anatomy of Threats to the Internet of Things. IEEE Commun. Surv. Tutorials 2019, 21, 1636-1675. [CrossRef]

20. Hassija, V.; Chamola, V.; Saxena, V.; Jain, D.; Goyal, P.; Sikdar, B. A Survey on IoT Security: Application Areas, Security Threats, and Solution Architectures. IEEE Access 2019, 7, 82721-82743. [CrossRef]

21. Newsome, J.; Shi, E.; Song, D.; Perrig, A. The Sybil attack in sensor networks: Analysis \& defenses - IEEE Conference Publication. In Proceedings of the Third International Symposium on Information Processing in Sensor Networks, Berkeley, CA, USA, 27 April 2004.

22. El-hajj, M.; Fadlallah, A.; Chamoun, M.; Serhrouchni, A. A Survey of Internet of Things (IoT) Authentication Schemes. Sensors 2019, 19, 1141. [CrossRef] [PubMed]

23. Frustaci, M.; Pace, P.; Aloi, G.; Fortino, G. Evaluating critical security issues of the IoT world: Present and future challenges. IEEE Internet Things J. 2018, 5, 2483-2495. [CrossRef]

24. Moustafa, N.; Turnbull, B.; Choo, K.K.R. An ensemble intrusion detection technique based on proposed statistical flow features for protecting network traffic of internet of things. IEEE Internet Things J. 2019, 6, 4815-4830. [CrossRef]

25. Clarence, C.; David, F. Machine Learning and Security [Book]; O'Reilly Media, Inc.: Newton, MA, USA, 2018.

26. Hodo, E.; Bellekens, X.; Hamilton, A.; Dubouilh, P.L.; Iorkyase, E.; Tachtatzis, C.; Atkinson, R. Threat analysis of IoT networks using artificial neural network intrusion detection system. In Proceedings of the 2016 International Symposium on Networks, Computers and Communications, ISNCC 2016, Yasmine Hammamet, Tunisia, 11-13 May 2016.

27. Moteiv Corporation Tmote Sky-Ultra Low Power IEEE 802.15.4 Compliant Wireless Sensor Module. 2006. Available online: http:/ / www.crew-project.eu/sites/default/files/tmote-sky-datasheet.pdf (accessed on 5 December 2020).

28. Wireshark Go Deep. Available online: https://www.wireshark.org/ (accessed on 28 November 2020).

29. Amirinasab Nasab, M.; Shamshirband, S.; Chronopoulos, A.; Mosavi, A.; Nabipour, N. Energy-Efficient Method for Wireless Sensor Networks Low-Power Radio Operation in Internet of Things. Electronics 2020, 9, 320. [CrossRef] 
30. Bandekar, A.; Javaid, A.Y. Cyber-attack Mitigation and Impact Analysis for Low-power IoT Devices. In Proceedings of the 2017 IEEE 7th Annual International Conference on CYBER Technology in Automation, Control, and Intelligent Systems, CYBER 2017, Honolulu, HI, USA, 31 July-4 August 2018; pp. 1631-1636.

31. Amir Alavi, S.; Rahimian, A.; Mehran, K.; Alaleddin Mehr Ardestani, J. An IoT-Based Data Collection Platform for Situational Awareness-Centric Microgrids. In Proceedings of the Canadian Conference on Electrical and Computer Engineering, Quebec City, QC, Canada, 13-16 May 2018; Volume 2018. 